\title{
TU/e emonowne

\section{Gas-phase plasma synthesis of free-standing silicon nanoparticles for future energy applications}

\section{Citation for published version (APA):}

Doğan, I., \& van de Sanden, M. C. M. (2016). Gas-phase plasma synthesis of free-standing silicon nanoparticles for future energy applications. Plasma Processes and Polymers, 13(1), 19-53.

https://doi.org/10.1002/ppap.201500197

DOI:

10.1002/ppap.201500197

Document status and date:

Published: 01/01/2016

\section{Document Version:}

Publisher's PDF, also known as Version of Record (includes final page, issue and volume numbers)

\section{Please check the document version of this publication:}

- A submitted manuscript is the version of the article upon submission and before peer-review. There can be important differences between the submitted version and the official published version of record. People interested in the research are advised to contact the author for the final version of the publication, or visit the $\mathrm{DOI}$ to the publisher's website.

- The final author version and the galley proof are versions of the publication after peer review.

- The final published version features the final layout of the paper including the volume, issue and page numbers.

Link to publication

\section{General rights}

Copyright and moral rights for the publications made accessible in the public portal are retained by the authors and/or other copyright owners and it is a condition of accessing publications that users recognise and abide by the legal requirements associated with these rights.

- Users may download and print one copy of any publication from the public portal for the purpose of private study or research.

- You may not further distribute the material or use it for any profit-making activity or commercial gain

- You may freely distribute the URL identifying the publication in the public portal.

If the publication is distributed under the terms of Article 25fa of the Dutch Copyright Act, indicated by the "Taverne" license above, please follow below link for the End User Agreement:

www.tue.nl/taverne

Take down policy

If you believe that this document breaches copyright please contact us at:

openaccess@tue.nl

providing details and we will investigate your claim. 


\title{
Gas-Phase Plasma Synthesis of Free-Standing Silicon Nanoparticles for Future Energy Applications
}

\author{
İlker Doğan, ${ }^{*}$ Mauritius C. M. van de Sanden*
}

Silicon nanoparticles (Si-NPs) are considered as possible candidates for a wide spectrum of future technological applications. Research in the last decades has shown that plasmas are one of the most suitable environments for the synthesis of Si-NPs. This review discusses the unique size-dependent features of Si-NPs, and the fundamental mechanisms of nanoparticle formation in plasmas by highlighting major plasma synthesis techniques. In addition, the routes to achieve control on Si-NP morphology and chemistry in plasma environments will be discussed. We will review recent advancements in solar cell and lithium-ion battery applications of gas-phase plasma synthesized Si-NPs by highlighting key results from the literature. We will

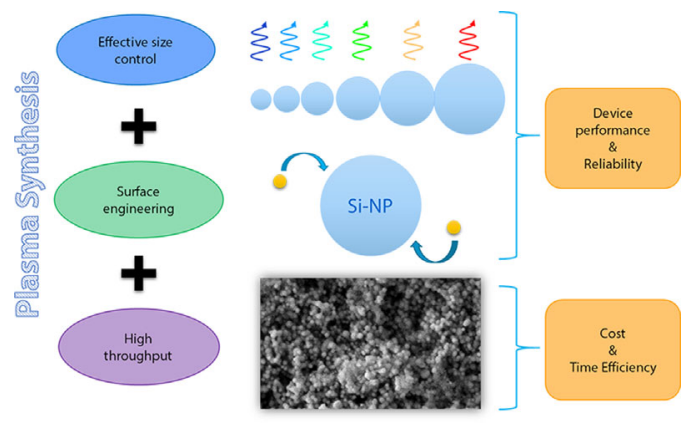
discuss further technological applications, where gasphase plasma synthesized Si-NPs can contribute, like water splitting and thermoelectrics.

\section{Introduction}

Crystalline silicon (c-Si) is one of the most important material in modern technology era, which is presently exploited in the microelectronics and the photovoltaics industry. It will probably continue to be the backbone material of the future technological applications for years to come. An important reason for $c$-Si being as a widely used material is its abundance -although Si is rarely found in pure elemental form in the nature, it is the eighth most common element in the universe and the second most common element in the Earth's crust in terms of mass. Si

i. Doğan, M. C. M. van de Sanden

Dutch Institute for Fundamental Energy Research (DIFFER),

P.O. Box $63365600 \mathrm{HH}$ Eindhoven, The Netherlands

Department of Applied Physics, Plasma and Materials Processing

Group, Eindhoven University of Technology, P.O. Box 5135600

MB Eindhoven, The Netherlands

E-mails: i.dogan@tue.nl; m.c.m.vandesanden@differ.nl can be easily purified, it is nontoxic, and its natural oxide, i.e., $\mathrm{SiO}_{2}$ is a good matching insulator in device applications. For microelectronic applications, the flexibility to control its conductivity effectively, and to dope it with the groups III and IV elements, and the possibility to produce monocrystal $\mathrm{Si}(c-\mathrm{Si})$ on a large scale are important advantages. In addition, having an optical bandgap in the NIR-visible region at $\sim 1.12 \mathrm{eV}, c-\mathrm{Si}$ is a suitable material for optical absorption, and can therefore be used in photovoltaic applications. However, the optical emission process efficiency is dramatically hindered as a result of the indirect bandgap structure of $c$-Si. Therefore, $c$-Si is an inefficient light emitter.

In July of 1990, Leigh T. Canham, a British physicist, reported visible (red) room temperature photoluminescence (PL) that is observable with naked eye from nanostructured porous silicon. In his work, he has asserted that fabricating silicon structures small enough exploits quantum size effects on the bandgap structure. He introduced this novel concept as "bandgap engineering." 
When compared to the defect engineering, which is also used to improve the optical properties of $c$-Si by creating impurity-activated radiative transitions, ${ }^{[1-4]}$ bandgap engineering favors visible PL from Si via increasing the efficiency of band edge emission. Since then, luminescent silicon nanoparticles (Si-NPs) have received a significant consideration for a wide range of optical, and light emitting applications, thanks to their size-dependent PL with high quantum yield.

Apart from the improved optical properties, Si-NPs are observed to exhibit novel physical and chemical properties, such as improvements in their dielectric constants, charge storage capacities, and catalytic activities, which reveals the potential in application areas of Si-NPs in future technologies. Particularly, Si-NPs can be used as visible light emitters in light emitting diodes (LEDs), ${ }^{[5-10]}$ charge storing elements in non-volatile memories ${ }^{[5,11-13]}$ and high capacity Li-ion batteries, ${ }^{[14-17]}$ catalyzers in water splitting applications for hydrogen production, ${ }^{[18,19]}$ biomarkers in tissue imaging, ${ }^{[20,21]}$ photocurrent generator,s and spectrum converters in solar cells. ${ }^{[22-25]}$ Among the abovementioned applications, Si-NPs are particularly studied in energy production and storage applications as the potential impact of Si-NPs in these areas might bring substantial change to realize a sustainable future.

In the context of realizing the above-mentioned future applications using Si-NPs, and establishing efficient and reliable device performances, well-controlled, scalable, and cost-efficient processing techniques are needed. As the Si-NP size, morphology, and surface chemistry substantially influence its optical and electronic properties (and therefore the device performance), realizing an effective nanoparticle size control and surface engineering is required. Establishing these requirements are sufficient for controlled synthesis of Si-NPs in the lab-scale, however, for promoting Si-NP synthesis to industrial scale, a high throughput processing and easy manipulation of synthesized Si-NPs during post-processing are necessary (Scheme 1).

Si-NPs are synthesized by using several methods, which include physical and chemical vapor deposition (PVD and CVD), ${ }^{[11,26-29]}$ ion radiation, ${ }^{[30-32]}$ and solution synthesis routes. ${ }^{[33-35]}$ The common drawback of most of the PVD, CVD, and ion radiation techniques is that, a post annealing procedure is necessary for inducing nanoparticle formation, which is an additional step that brings increased amount of time and cost. Apart from that, produced Si-NPs are embedded in matrices, making them unavailable for easy handling and manipulation. For instance, in order to obtain Si-NPs free from matrices, the surrounding medium must be removed by means of etching, bringing also an additional post-production step. Chemical solution synthesis routes have a major advantage of producing sizecontrolled, luminescent, free-standing Si-NPs. However,

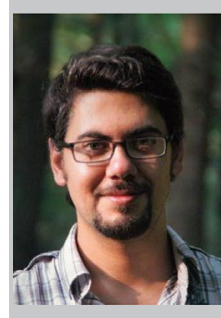

Dr. ilker Doğan received his M.S. degree in physics from the Middle East Technical University, Ankara, Turkey, in 2008, and Ph.D. degree from Eindhoven University of Technology, Eindhoven, The Netherlands, in 2014. From June 2005 till September 2009, he was a researcher in SEMINANO FP-6 Project, and a research and teaching assistant at Middle East Technical University. Between September 2009 and October 2013 he was a researcher in Joint Solar Programme of Foundation for Fundamental Research on Matter (FOM), The Netherlands. After having worked for ASML Netherlands B.V. as a research scientist for 1 year, he joined back to academia as a postdoctoral research fellow at the Dutch Institute for Fundamental Energy Research (DIFFER), and Eindhoven University of Technology. His main research interests are synthesis and formation of nanoparticles in plasmas, nanomaterial characterization, and sustainable energy applications of nanomaterials. Dr. Doğan was a finalist of the Coburn and Winters Award of the Plasma Science and Technology Division at the 59th American Vacuum Society Symposium in 2012, and the winner of the best oral presentation award of the Plasma Nanoscience Foundation at the International Plasma Nanoscience Symposium in 2013.

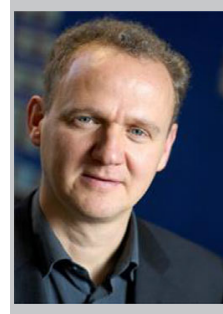

Prof. Dr. Mauritius C. M. van de Sanden received the Ph.D. degree on the topic of the fundamental investigation of an expanding plasma jet from the Eindhoven University, Eindhoven, The Netherlands, in 1991. His main research interests are fundamental investigation on plasmas, and plasma-surface interactions, in situ analysis and control of the physical and chemical properties of plasma-processed materials, and formation of $\mathrm{CO}_{2}$ neutral fuels (solar fuels) using sustainable energy sources. In 2000, he was appointed as a Full Professor at the Eindhoven University of Technology, Eindhoven. He is currently the Director of the Dutch Institute for Fundamental Energy Research and a Professor at the Department of Applied Physics, Eindhoven University of Technology. He has authored more than 400 papers in peer-reviewed journals and is the coinventor of more than 20 patents. He is a Fellow of AVS, IUPAC, and IOP. Since 2005 , he has been the European Associate Editor of Plasma Sources Science and Technology and he is on the editorial board of Plasma Processes and Polymers. In 2008, he has received the European William Crookes Plasma Prize for his midcareer achievements. In 2009, he received the Dutch Valorisation Prize for his achievements in transferring scientific knowledge to industry. Since 2013 he is a member of The Netherlands Academy of Arts and Sciences and in $\mathbf{2 0 1 4}$ he won the Plasma Prize of the AVS Plasma Science and Technology Division.

these techniques require extensive reaction time with multiple production steps still with low yields. Moreover, the chemical compounds that are used for Si-NP synthesis are generally complex molecules, which brings along 


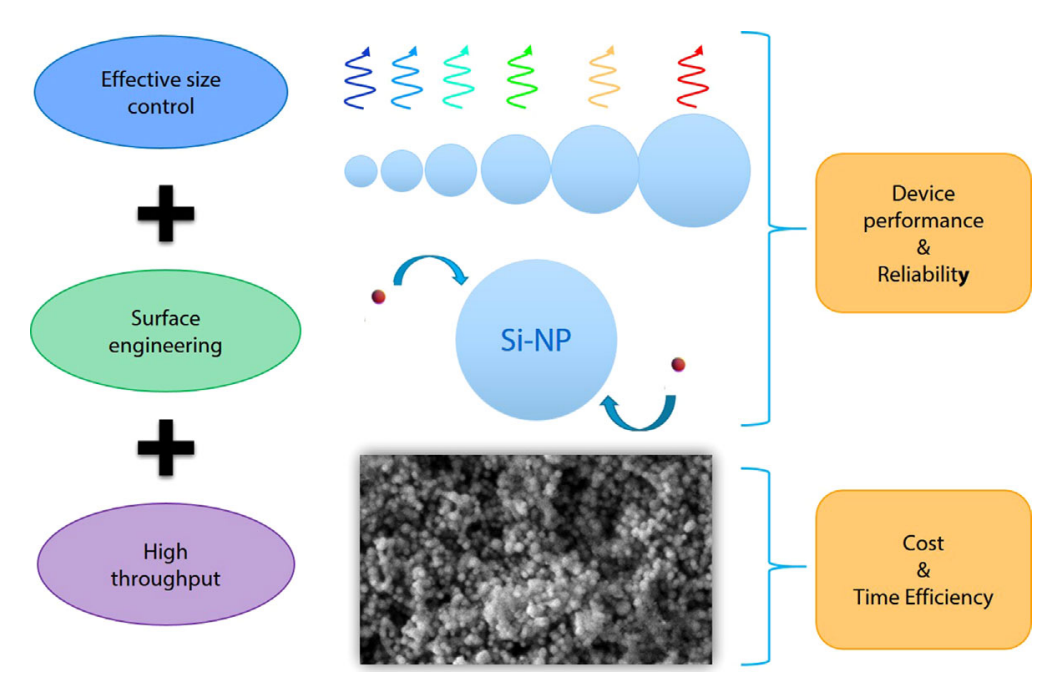

Scheme 1. Requirements for realistic applications of Si-NPs include effective size control, surface engineering, and high throughput processing. The first two requirements are critical for device performance and reliability while the latter is critical for cost and time efficiency of the Si-NP production process. battery applications. We will first review the size-dependent properties of Si-NPs, i.e., bandgap, surface-to-volume ratio, surface reactivity, and thermal conductivity, and their importance to the energy related applications. Later, we will review the growth models and functionalization of Si-NPs in gas-phase plasma-chemical environments. After that, we will give a literature review on the photovoltaic and battery applications of Si-NPs. We will also discuss on the hydrogen production and thermoelectric applications, where plasmas yet to be involved, that can potentially benefit from the plasma synthesis routes in the sense of throughput and surface functionalization. Finally, we will discuss the advantages, and challenges of the plasma synthesis of Si-NPs, and their applications to energy technologies, and draw conclusions. specialized expensive facilities during precursor production. The most important issue of these above-mentioned techniques are their scalability: these techniques are mostly compatible to lab-scale production with relatively low throughputs, hampering large scale applications of Si-NPs using these technologies (Scheme 2).

Gas-phase plasma-chemical synthesis tools are considered to have great potential on the production of Si-NPs in a controlled and efficient way. Compared to the other Si-NP synthesis methods, gas-phase plasma-chemical processing techniques have unique advantages like one-step production of free-standing Si-NPs, the feasibility of promoting high throughput synthesis, the flexibility of using the precursor material in any desired form (i.e., solid, liquid, or gas form), and the freedom of modifying the material morphology and surface chemistry by taking advantage of tunable plasma parameters. As an example, non-thermal plasmas, ${ }^{[36-43]}$ multi-hollow discharges, ${ }^{[44,45]}$ and remote expanding thermal plasmas ${ }^{[46-48]}$ have been reported as suitable synthesis techniques of free-standing Si-NPs using various precursors, where in situ surface functionalization $^{[36,37,49-52]}$ and morphological modification via in situ etching ${ }^{[53-55]}$ are also possible. Higher throughputs up to $\sim 100 \mathrm{mg} \mathrm{min}^{-1}$ are reported in lab-scale gas-phase plasma processing tools, ${ }^{[46]}$ which is already in the order of fabscale production expectations for technological applications. Therefore, these methods could be considered as a step forward to promote large scale production (Scheme 2).

In this paper, we will review the recent achievements of plasma-chemical synthesis environments on the synthesis of free-standing Si-NPs and composite nanoparticles including $\mathrm{Si}$ that are mainly used for photovoltaic and

\section{Size-Dependent Properties of Si-NPs and Their Relevance to the Future Energy Technologies}

\subsection{Size-Dependent Bandgap}

Nanostructured silicon (and also other nanostructured materials) is classified with respect to its dimensionality, where charge carries (electrons and holes) are free to move. In the dimensions that are reduced to nanometer scale, charge carriers are confined and intrinsic optical and

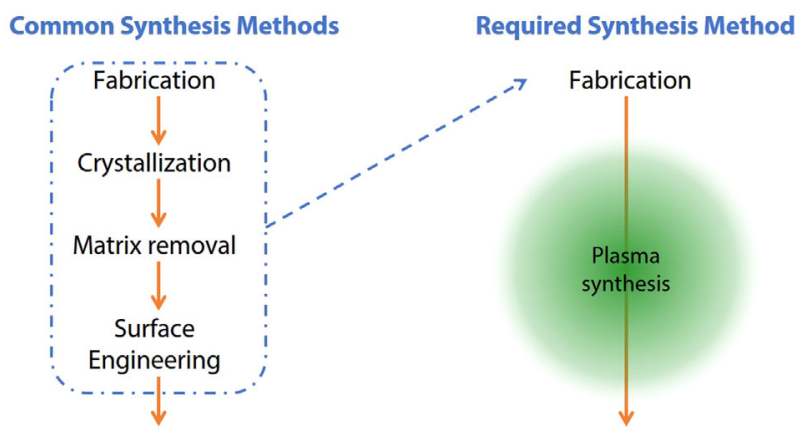

Free Standing Si-NPs

Free Standing Si-NPs

Scheme 2. A typical production route for the synthesis of freestanding Si-NPs: in most of the thin film deposition techniques, as-synthesized films containing excess $\mathrm{Si}$ is annealed to induce Si-NP formation. After that, this matrix is removed via etching and surface functionalization is performed on free-standing SiNPs. An idealized synthesis technique should realize one-step production of free-standing Si-NPs with an in situ functionalization possibility. 
electronic properties exhibit size-dependent features. Depending on the degree of free dimensions, these nanostructures are classified as 2D quantum wells, 1D quantum wires, or nanowires, and OD quantum dots, or nanoparticles. Therefore, in Si-NPs, charge carriers are confined in all physical dimensions. Particularly, when the size of a Si-NP becomes smaller than its exciton-Bohr radius, $a_{\mathrm{B}-\mathrm{Si}}$ $\sim 5.3 \mathrm{~nm}$, the intrinsic size of an exciton in bulk $c-\mathrm{Si}_{1}{ }^{[56]}$ the energy band diagram of $\mathrm{Si}$ is discretized. In this case, electrons and holes do not have bound states in the $\boldsymbol{k}$-space, which makes them behave similarly to hydrogen-like excitons. This allows phonon-free optical transitions ${ }^{[57]}$ as a result of increased oscillator strength of "no-phonon" transition process. ${ }^{[58]}$ The band-to-band (between the edges of valence band, $\mathrm{VB}$ and the conduction band, $\mathrm{CB}$ ) absorption energy in a Si-NP is described as follows:

$$
E_{n l}=E_{g}+\frac{\hbar^{2} \chi^{2} n l}{2 \mu a^{2}}
$$

where $E_{\mathrm{g}}$ is the bandgap energy of bulk $c$-Si, and the second term at the right-hand-side is the quantized exciton energy ( $\chi$ describes the quantization of energy levels, i.e., $\chi=1,2,3, \ldots$, $\mu$ is the reduced mass of the exciton, and $\alpha$ is the nanoparticle size). $n$ and $l$ are the quantum numbers that describe the Coulomb electron-hole interactions (1S, 2S, 2P, ...), and the states connected within the exciton center of mass motion $(1 s, 2 s, 2 p, \ldots)$ under the effect of external conditions, respectively. As Equation 1 clearly demonstrates, the bandgap energy $E_{n l}$, or $E_{\mathrm{g} \text { Si-NP, }}$ has a strong size dependency. Together with the enhanced radiative transition rate, the size-dependent bandgap of Si-NPs not only fosters room temperature visible luminescence, but also enables the color tuning of the luminescence by simply varying its size. ${ }^{[59]}$ This unique feature of Si-NPs with respect to its bulk counterpart promotes the use of Si-NPs in optical and optoelectronic applications. In the case of photovoltaic applications, the size-dependent bandgap of Si-NPs enables absorption of light from various parts of solar spectrum, mostly in the visible range. However, different from optical applications, generated charge carriers upon absorption of solar radiation have to be separated before they recombine, in order to generate current. Various photoluminescence spectroscopy (PL) analyses demonstrate that Si-NPs exhibit size and/ or surface chemistry dependent absorption/emission in the visible spectrum, which can be tailored by varying the Si-NP size and/or functionalizing the Si-NP surfaces with ligands. ${ }^{[57,60-69]}$ For an in depth information on the interplay bandgap energy. between quantum size effects and surface effects on the luminescence properties of Si-NPs we suggest the review of Mariotti et al. ${ }^{[70]}$

\subsection{Advanced Photon Management}

A remarkable feature of Si-NPs (and in other semiconductor nanoparticle systems) is the enhancement in electron-hole pair multiplication due to the increased rate of phonon-free transitions, modified carrier-cooling rates, and enhanced Coulomb coupling between charge carriers. ${ }^{[57,71-80]}$ From the perspective of photovoltaic applications, these features can potentially enable "advanced photon management" concepts of "hot" photons, originating from the high energy window of the solar spectrum, via multi-exciton generation (MEG) processes. MEG in Si-NPs is the counterpart of impact ionization in bulk $c$-Si, however with a significantly increased rate. ${ }^{[71]}$ In the past decade, MEG has been confirmed in several semiconductor nanoparticle systems, such as InAs, ${ }^{[81]} \mathrm{InP}^{[82]} \mathrm{CdSe}^{[83]} \mathrm{PbTe}^{[84]} \mathrm{PbS}^{[85]}$ $\mathrm{PbSe}^{[83,85,86]}$ and SiGe ${ }^{[87]}$ as well as Si-NPs. ${ }^{[57,71-75,80]}$

MEG can take place either within a single Si-NP ${ }^{[72]}$ or between adjacent Si-NPs. ${ }^{[80]}$ It is shown that, closely packed Si-NP ensembles can enable efficient carrier extraction via transferring the excess energy absorbed by a Si-NP to an adjacent Si-NP creating an additional exciton. This type of MEG is also known as "space separated quantum cutting" (SSOC), which provides longer lifetimes for the extraction of excitons. ${ }^{[7,75,79]}$ The external quantum yield of an ensemble of closely packed Si-NPs as a function of the excitation energy for a constant number of photons is a direct measure of carrier multiplication efficiency. In principle, the external quantum yield increases by $100 \%$ of the initial efficiency for each multiples of the bandgap energy as seen in Figure 1a.
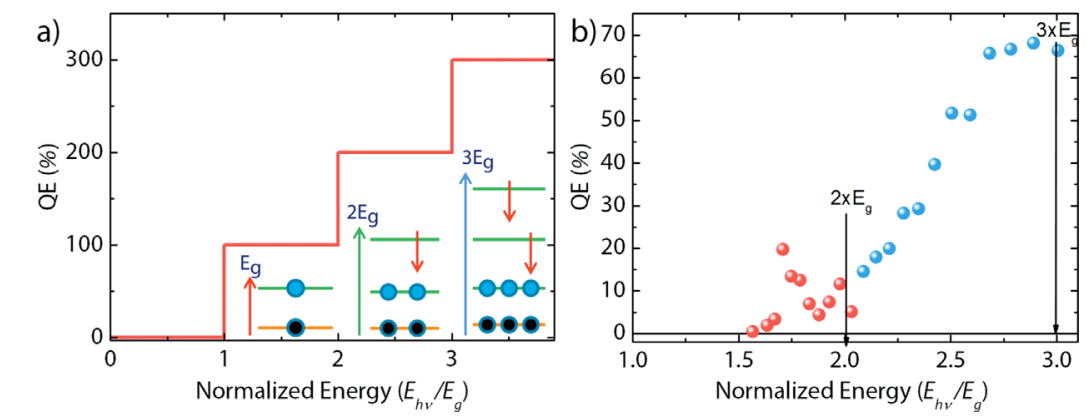

Figure 1. (a) MEG as a function of normalized energy where $E_{\mathrm{h} v}$ is the incoming photon energy and $E_{\mathrm{g}}$ is the bandgap energy of Si-NP. Blue dots represent the electrons and black dots represent the holes. (b) MEG between the Si-NPs synthesized in a remote expanding thermal plasma. The reason of not observing a step-like enhancement in quantum efficiency is due to the broad size distribution of Si-NPs ranging from 2-120 nm. Presence of very large Si-NPs also hinders the overall quantum efficiency, reaching around $70 \%$ even after exciting Si-NPs with energies three times of the 
However, this enhancement can be affected by the size distribution, surface quality, and optical properties of Si-NPs, resulting in lower efficiency enhancements than the ideal case as we observed from remote expanding thermal plasma synthesized Si-NPs (Figure 1b). MEG process observed from Si-NPs finds an important application in photovoltaics in the form of "down-converters," where the photons from high energy part of the solar spectrum are converted into multiple low energy photons, matching to the bandgap energy of the solar cell junction material. An important advantage of Si-NP down-converters is that, as the down-converter is a passive layer (it does not require electrical contact with the solar cell), it can be integrated into the encapsulation material of solar cell devices.

\subsection{Increased Surface-to-Volume Ratio}

\subsubsection{Increased Diffusion Coefficient}

Apart from the improved optical properties, reduction in size increases the surface-to-volume ratio, which brings additional advantages on the use of Si for energy related applications. For instance, reduction in size reduces the required diffusion distances and increase diffusion coefficients of atomic or ionic species through the nanoparticle, enhancing the mass transport rate. ${ }^{[88]}$ Moreover, mechanical stability and the ability to reduce facile strain at nanometer scale prevents crack formation and propagation through the interior of Si-NPs, ${ }^{[89,90]}$ which makes them morphologically stable against their bulk counterparts upon volumetric changes due to the high density of diffusing species, and alloy formation with diffusants. These advantages of Si-NPs at nanoscale finds application in energy storage, as an active anode material in lithium-ion batteries -making use of the ten-times higher capacity of silicon $\left(4000 \mathrm{mAh}^{-1}\right)$ with respect to commercial graphite counterparts $\left(375 \mathrm{mAh}^{-1}\right){ }^{[91]}$

\subsubsection{Increased Surface Reactivity}

Silicon is a covalent solid, its surface reactivity is mostly determined by unsatisfied valences. ${ }^{[92]}$ In the case of Si-NPs, reconstruction of the surface leaves dangling bonds, which aggressively react with the surrounding environment. As a result of increased surface-to-volume ratio of Si-NPs, the chemical state of the surface plays a critical role in the overall chemical reactivity of Si-NPs. Consequences of this reactivity at first sight seem detrimental mostly because of unwanted surface-chemical modifications, such as uncontrolled oxidation of bare Si-NP surfaces in air, ${ }^{[26,68,93,94]}$ or ligand attachments during solution processing, ${ }^{[95-98]}$ or irreversible passive layer formation in some electrochemical reactions ${ }^{[99,100]}$-all limiting the expected optical, electrical, and chemical performance of Si-NPs. This is, however, not always the case. Si-NPs can be used as a chemical splitting agent by virtue of their surface reactivity, and undergo chemical reactions, where the byproducts are relevant for energy related applications. For instance, oxidation of Si-NP surfaces by water releases hydrogen gas, ${ }^{[18,101-104]}$ used for energy storage, catalysis, and fuel cell applications. ${ }^{[105-108]}$ This so-called "hydrogen production via splitting of water" process using Si-NPs has a high chance of becoming an increasingly more interesting research topic as a result of very high efficiency and environmental compatibility of the process $\left(\mathrm{CO}_{2}\right.$ - and toxic product-free process). As expected, the hydrogen gas production rate increases with scaling-down of Si structure size from micrometer range to nanometer range, ${ }^{[18]}$ stressing the benefit of increased surface area at nanoscales.

\subsection{Reduced Thermal Conductivity}

Downscaling the size of Si in the nanometer range also has a size-dependent impact on the lattice thermal conductivity and electron thermal conductivity. In a crystal, phonons usually have longer mean-free path (up to several hundreds of nanometers) than the electron mean-free path (up to several nanometers). ${ }^{[109]}$ If the size of a Si-NP is adjusted in between the phonon and electron mean-free paths, the phonon scattering rate increases, reducing the thermal conductivity, and the electron mean-free path remains unchanged. ${ }^{[110]}$ This feature opens the way for thermoelectric applications, a thermo-voltage generation process (based on the Seebeck effect) from the two ends of an electrically conductive material, which are exposed to different temperatures. Using Si-NPs in thermoelectric applications can potentially improve waste heat recovery from automobile exhausts, and from industrial processes. ${ }^{[111]}$ It is observed that, size-dependent reduction in the thermal conductivity increases the dimensionless figure of merit $Z T=S^{2} \sigma T / k$ ( $S$ is the seebeck coefficient, $\sigma$ is the electrical conductivity, $k$ is the thermal conductivity, and $T$ is the absolute temperature) of Si-NPs from $\sim 0.2$ to $\sim 0.7$ at $1280 \mathrm{~K}^{[112]}$ Although this value is still lower than the state-of-the-art $Z T$ values of $\sim 2.4$ from $\mathrm{Bi}_{2} \mathrm{Te} / \mathrm{Sb}_{2} \mathrm{Te}_{3}$ supperlattices, ${ }^{[113]}$ environmental compatibility, abundance, and affordability of Si-NPs brings them to the focus of the thermoelectric research.

\section{Synthesis and Functionalization of Si-NPs in Plasmas}

Gas-phase formation of Si-NPs in plasmas has been observed in so-called "dusty plasma" conditions. Their first observation in the plasmas did not get much attention as a technologically relevant material. They were commonly 
approached as unwanted inclusions during the processing of amorphous silicon ( $a-\mathrm{Si}: \mathrm{H}$ ) thin films as they were mostly observed to deteriorate the electrical and optical quality of the deposited films. After their unique size-dependent physical and chemical features were realized, plasma scientists put special attention on the growth and control of Si-NPs in plasmas. In this section we will review the synthesis and functionalization of free-standing Si-NPs in various plasma environments.

\subsection{Synthesis}

Plasmas that are used to synthesize Si-NPs in the gas-phase are classified in six groups in this review. These are so-called atmospheric pressure microplasmas, non-thermal plasmas, capacitive high frequency discharges, microwave plasmas, remote plasmas (expanding thermal plasmas), and thermal plasmas. These plasmas vary in their sizes, volumes, power coupled to them, and processing rate of Si-NPs. As seen in Figure 2, the processing rate of Si-NPs can be from $10 \mathrm{mg} \mathrm{h}^{-1}$ (using microplasmas) up to $400 \mathrm{gh}^{-1}$ (using thermal plasmas). Apart from their common advantages with respect to non-plasma based synthesis routes, all of these plasma processing techniques have their own advantages and disadvantages with respect to each other. For instance, microplasmas and non-thermal plasmas have relatively low throughput due to their limited plasma volume of small dimensions. However, this limited plasma volume brings an advantage of improved size distribution control during Si-NP processing, which is generally considered as a challenge to achieve for the large volume, high power, high throughput plasmas. On the other hand, the high

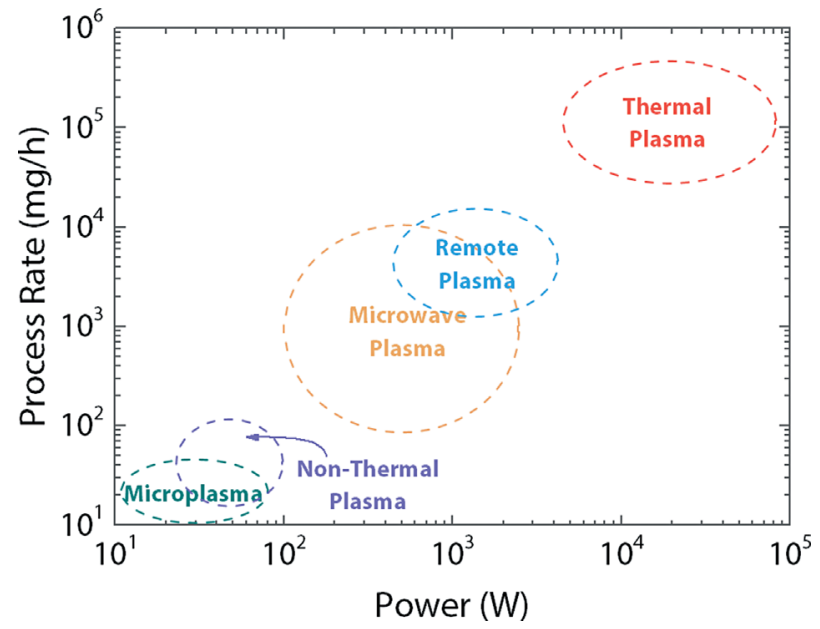

Figure 2. Demonstration of Si-NP processing rate of various gasphase plasma synthesis tools as a function of power. In general the higher the source power, the higher the throughput of Si-NPs. Depending on the plasma technique used, the processing rate can be from tens of milligrams per hour to hundreds of grams per hour. processing rate plasmas are compatible for large scale applications, and further scaling-up is much more feasible with respect to plasmas with smaller dimensions. In this section, we will briefly review the characteristics, and nanoparticle growth kinetics of the plasmas demonstrated in Figure 2.

\subsubsection{Non-Thermal Plasmas}

Non-thermal plasmas are characteristically composed of high energy "hot" electrons between 2 and $5 \mathrm{eV}$, and significantly colder ions and neutral species, mostly close to the room temperature. Non-thermal plasmas are usually generated using a gas mixture of $\mathrm{Ar} / \mathrm{SiH}_{4}{ }^{[36,42,49,114-117]}$ or

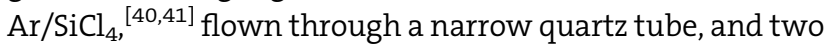
copper rings are used as electrodes to couple the RF power of several tens of Watts to the gas mixture to generate a plasma (Figure 3). Non-thermal plasma is partially ionized, which

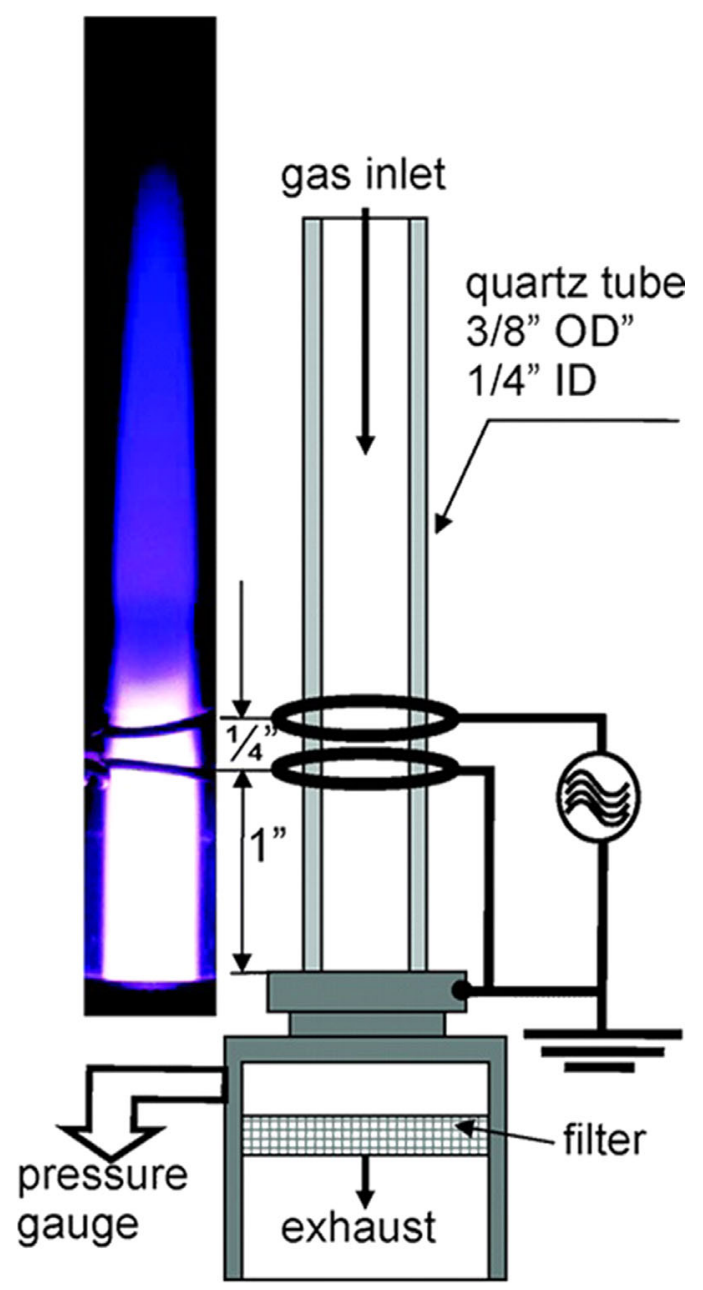

Figure 3. Schematic of the non-thermal plasma system with an image of the plasma during operation. (Reprinted with permission from Mangolini et al. ${ }^{[115]}$ Copyright 2005, American Chemical Society.) 
consists of ions, radicals, and neutral species. Hot electrons in the non-thermal plasma environment dissociate the precursor gas leading to the formation of mainly negative ions, and radicals. In non-thermal plasmas, Si-NP formation is mainly driven by anion-neutral reactions ${ }^{[114]}$ Nanoparticle formation starts with polymerization reactions driven by negative ions -the species that are trapped in the plasma due to the negatively charged walls -leading to the formation of $\mathrm{Si}_{n} \mathrm{H}_{m}$ clusters. As a result of the high electron affinity of the clusters, and the high mobility of the electrons in the plasma, emerging clusters have a tendency to become negatively charged. ${ }^{[114,118]}$ Furthermore, chemical kinetics models from the group of Kortshagen demonstrated the importance of two anion-neutral clustering channels, involving negative silyl $\left(\mathrm{Si}_{n} \mathrm{H}_{2 n+1}{ }^{-}\right)$and silylene $\left(\mathrm{Si}_{n} \mathrm{H}_{2 n}{ }^{-}\right)$species, ${ }^{[119,120]}$ respectively. Once negatively charged ionic clusters appear in the plasma, they act as seeds for nucleation. Further Si-NP growth then continues via addition of ions, radicals, and neutral species to the cluster surface. Si-NP size control in non-thermal plasmas is achieved by tuning their residence times in the plasma, which is generally less than $10 \mathrm{~ms} .{ }^{[40,115]}$ This yields a narrow size distribution of SiNPs within the range of $2-20 \mathrm{~nm}$. In addition, partial pressure of the precursor is also observed to increase the size of the synthesized Si-NPs. ${ }^{[115]}$

Si-NPs formed in the non-thermal plasmas can be tuned in morphology, i.e., amorphous and fully crystalline Si-NPs can be produced, by means of controlling the power coupled into the plasma. ${ }^{[40,117]}$ Crystallinity of Si-NPs is achieved via free electrons and ions combining at the nanoparticle surface, which excessively heats up the nanoparticle surfaces close to the melting point of Si while the gas

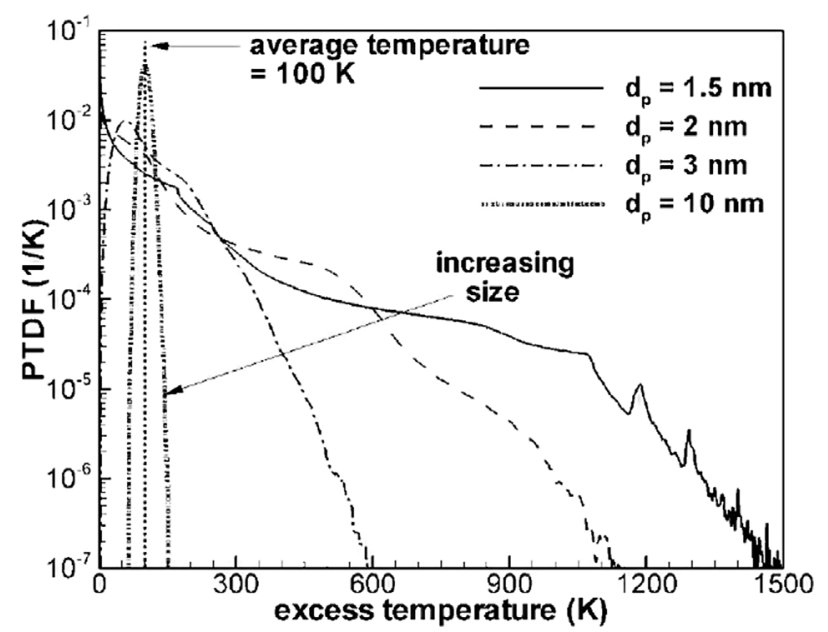

Figure 4. The distribution of nanoparticle temperatures for various Si-NP sizes. Average temperature is independent of the nanoparticle temperature and it has a value of $100 \mathrm{~K}$. (Reprinted figure with permission from Mangolini et al. ${ }^{[121]}$ Copyright 2009, American Physical Society.) remains close to the room temperature, see Figure $4 .{ }^{[121]}$ This unique feature makes non-thermal plasmas suitable synthesis environments of group III-V and group IV semiconductors with high melting points, which is not achievable with solution-based processes. In addition, nonthermal plasmas do not have the issue of particle agglomeration, thanks to unipolar negative charging of Si-NPs, suppressing hard agglomeration and prevent sintering individual Si-NPs. ${ }^{[14,122]}$ Mangolini et al. ${ }^{[115]}$ reported the processing rate of non-thermal plasma as $14.4 \mathrm{mg} \mathrm{h}^{-1}$ for small Si-NPs with sizes of $2-4 \mathrm{~nm}$, and as $52 \mathrm{mg} \mathrm{h}^{-1}$ for larger Si-NPs. Apart from Si-NPs, non-thermal plasmas are suitable for synthesizing other nanomaterials such as Ge-NPs, ${ }^{\left[{ }^{39,123]}\right.}$ SiGe-NPs, ${ }^{[124]}$ and SiC-NPs. ${ }^{[125,126]}$

\subsubsection{Capacitive High Frequency Discharges}

Capacitive high frequency discharges (CHF) have been used mostly for deposition of high quality amorphous silicon films ( $a$-Si:H) with high rate. The first observation of nano/ micro particles in $\mathrm{SiH}_{4}$ CHF discharges dates back to $1985{ }^{[127]}$ Depending on the processing conditions, dusty plasmas regime observed in CHFs, and they were considered as "unwanted" at that times as the dust particles deteriorate the quality of deposited $a-\mathrm{Si}: \mathrm{H}$ films. CHFs consists of parallel plates with a gap between them, where the plasma is generated by applying a high frequency power between these plates. The applied power scales with the area of the electrodes, and for the reported studies from various groups, a typical CHF system operates in a power range from a few $\mathrm{mW} \mathrm{cm}^{-2}$ to $1 \mathrm{~W} \mathrm{~cm}^{-2}$, applied on the electrodes. ${ }^{[128-130]} \mathrm{SiH}_{4}$ is mostly used as the precursor gas for the formation of Si-NPs.

CHF discharges are another form of non-equilibrium plasmas, where the electron temperature easily exceeds $3 \mathrm{eV}$, while the heavy species remain relatively close to the room temperature. Therefore, dissociation of the precursor takes place via electron-induced chemistry. Various Si-NP formation mechanisms have been proposed in CHF plasmas. Systematic studies from the group of Watanabe and Shiratani suggested that there is a close correlation with the spatial density of short lifetime radicals $\left(\mathrm{SiH}_{2}, \mathrm{SiH}\right.$, and $\mathrm{Si}$ ), and the spatial density of higher order silanes -cting as the seeds for nanoparticle growth -indicating that the dominant Si-NP growth mechanism is radical-driven reactions while ion-neutral driven chemistry does not play a significant role ${ }^{[128,131-139]}$ (Figure 5). Bouchoule and Boufendi have studied the formation of Si-NPs in CHF $\mathrm{Ar} / \mathrm{SiH}_{4}$ plasmas experimentally and theoretically, and concluded that the role of negative ions are of critical importance on the formation of ionic clusters that will act as seeds for the growth of Si-NPs. ${ }^{[129,140-142]}$ Hollenstein and Howling have also observed using laser light scattering and ion mass spectroscopy that anion-neutral pathway is the 


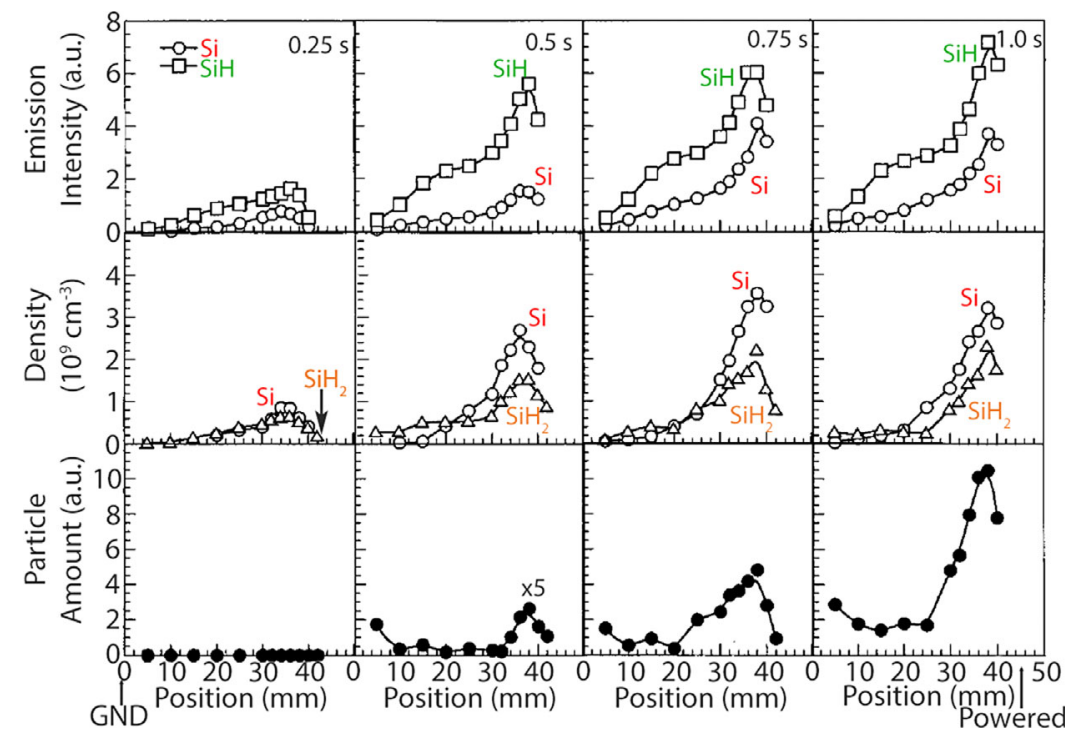

Figure 5. Spatial profiles of short lifetime radicals in the capacitive high frequency discharge. From top row to the bottom, graphs show $\mathrm{Si}$ and $\mathrm{SiH}$ emission intensities, $\mathrm{Si}$ and $\mathrm{SiH}_{2}$ density, and primary particle amount. (Reprinted with permission from Watanabe et al. ${ }^{[132]}$ Copyright 1996, American Vacuum Society.)

dominant Si-NP formation mechanism in the first growth phase. ${ }^{[130,143]}$ Although there are different conclusions on the dominant growth pathway of initially appearing clusters in CHF plasmas, the general growth mechanism follows similar steps: an initial growth phase for the formation of seed clusters and growth of these clusters from a few up to $10 \mathrm{~nm}$, a rapid growth phase, during which all the oppositely charged particles, and neutral particles undergo rapid coagulation, reaching sizes up to $100 \mathrm{~nm}$, and finally a growth saturation phase, where the remaining species (radicals, ions, and neutrals) contribute to the growth of Si-NPs via addition reactions on the surface of nanoparticles.

In CHF plasmas, Si-NP size control is achieved by tuning the RF on and off time. Increasing the RF on time increases the size of Si-NPS ${ }^{[128,144]}$ as it further drives the growth chemistry. In such kind of plasmas, Si-NP agglomeration could be observed depending on the RF on time, large agglomerates of hundreds of nanometers can be observed if the RF on time lasts for hundreds of milliseconds. ${ }^{[142]}$

\subsubsection{Atmospheric Pressure Microplasmas}

Microplasmas are atmospheric pressure gas discharges formed between electrodes, where at least one electrode dimension is less than $1 \mathrm{~mm}$. Microdischarges can be powered capacitively (Figure 6) ${ }^{[145,146]}$ or by direct current, ${ }^{[147]}$ with an electron temperature of up to $10 \mathrm{eV}$, and with a gas temperature of less than $0.2 \mathrm{eV}^{[145-147]}$ Formation of Si-NPs in microdischarges takes place via complete dissociation of the precursor gas in the high density reactive plasma -such as $\mathrm{SiH}_{4}{ }^{[147]}$ and $\mathrm{SiCl}_{4}{ }^{[145,146]}$ precursors -forming a supersaturated Si vapor. This supersaturated vapor of $\mathrm{Si}$ atoms undergoes a fast nucleation burst to form crystalline Si-NPs via three-body collision, ${ }^{[145,146]}$ and coagulation. ${ }^{[147]}$

In microdischarges, particle size control is achieved via tuning the residence time of the plasma, which can be varied from tens of microseconds up to $100 \mu \mathrm{s}$. Due to the limited dimensions of the plasma, Si-NPs can be synthesized with a very narrow size distribution, in average in the range $1-5 \mathrm{~nm}$ for Si-NPs. Apart from Si-NPs, formation of $\mathrm{Ni}$ and Fe nanoparticles, ${ }^{[148]}$ and functionalization of synthesized Si-NPs ${ }^{[70,149]}$ are also reported using microplasmas. The group of Sankaran demonstrated the use of microdischarge jets to synthesize metal nanoparticles in the concept of plasmaliquid electrochemistry via reduction of aqueous species, ${ }^{[148,150,151]}$ which shows great promise on improved size control, passivation, and long term storage of nanoparticles in aqueous media. A critical advantage of microdischarges over other plasma synthesis techniques is that the synthesis takes place under atmospheric pressure, and can potentially simplify implementation of the tool for direct applications, for example direct printing of Si-NPs on top of the already-finished solar cells as spectrum converters without a need of vacuum processing.

\subsubsection{Microwave Plasmas}

Microwave cavities are used as sources for decomposition of the precursor gas and plasma generation, and operates under high powers from hundreds to thousands of Watts, thus microwave plasmas are classified as thermal plasmas -where the gas temperature also exceeds $0.6 \mathrm{eV}$ besides the thermal electrons $\left(T_{\mathrm{e}}>1 \mathrm{eV}\right)$. One of the most successful demonstrations of Si-NP production, and functionalization in microwave plasmas is demonstrated by Wiggers and co-workers. ${ }^{[61,65,68,109,152]}$ They have used $\mathrm{Ar} / \mathrm{H}_{2} / \mathrm{SiH}_{4}$ gas mixture to synthesize crystalline Si-NPs with sizes from 3 to $30 \mathrm{~nm}$ (Figure 7). ${ }^{[68]}$ Formation of Si-NPs in microwave plasma is a homogeneous nucleation process from the supersaturated $\mathrm{Si}$ atoms. $\mathrm{As} \mathrm{SiH}_{4}$ is injected into the plasma zone, it is completely dissociated in a one-step process to form free $\mathrm{Si}$ atoms. These $\mathrm{Si}$ atoms condensate and nucleate to form primary particles during the flowthrough transport in the plasma. These initially formed small Si-NPs then sinter with each other and reach larger sizes. Once the grown Si-NPs are out of the plasma zone, the 
a)

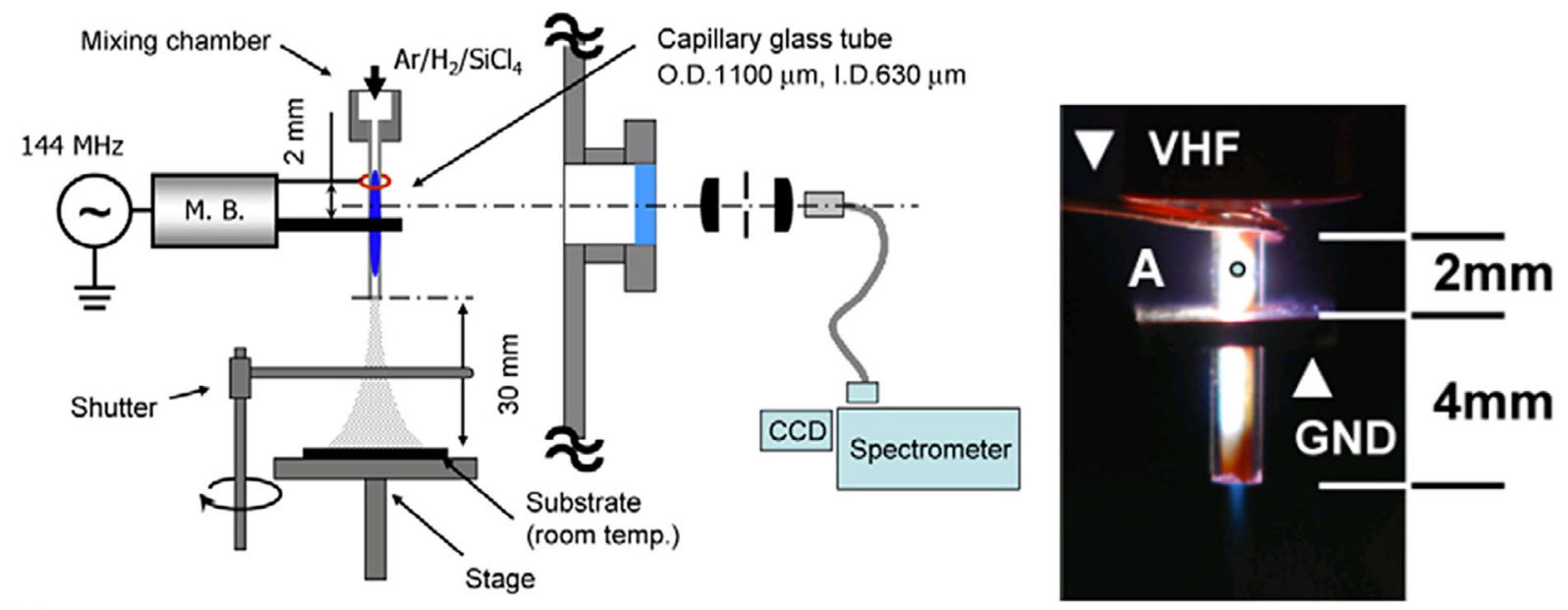

b)
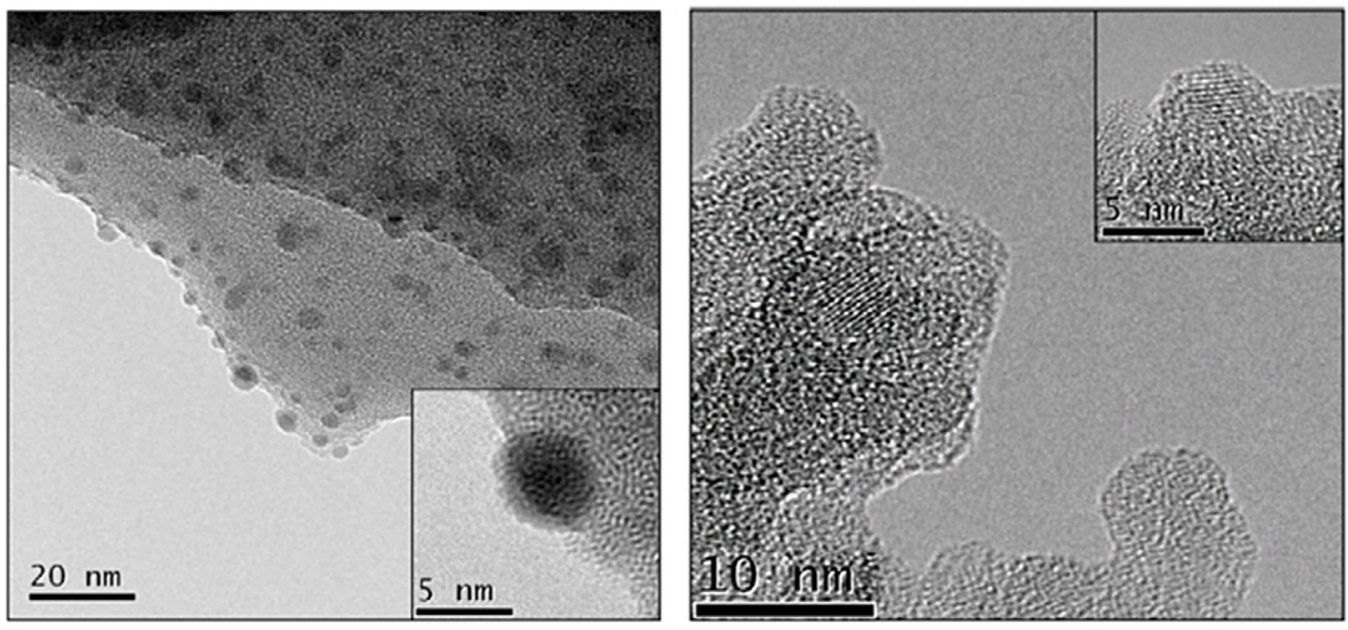

Figure 6. (a) Schematic diagram of a microplasma used for Si-NP synthesis, and (b) HRTEM images demonstrating Si-NPs synthesized in the microplasma. (Reprinted with permission from Nozaki et al. ${ }^{[15]}$ Copyright 2007, IOP Publishing. All rights reserved.)

gas temperature drops quickly and the characteristic sintering time for Si-NPs become longer with respect to the collision frequency with each other. Consequently, collisions between Si-NPs in the cold downstream zone does not grow larger Si-NPs but forms softly agglomerated Si-NP ensembles.

Size control of Si-NPs is realized by tuning the total gas pressure in the reaction chamber, $\mathrm{SiH}_{4}$ concentration, and the residence time. Increasing the total pressure, and $\mathrm{SiH}_{4}$ concentration increases the number of primary Si-NPs in the plasma, thus increasing their sintering rate, and growing larger Si-NPs. Residence time increase allows more time of the flow-through process for Si-NPs to grow before they leave the microwave plasma zone. Microwave induced flow-through plasmas are suitable environments for synthesizing Si-NPs with considerably higher throughputs -as high as $10 \mathrm{~g}$ of Si-NPs per hour of processing, ${ }^{[68]}$ which makes this type of plasma advantageous over the previously mentioned plasmas in terms of tackling the scalability challenges.

\subsubsection{Thermal Plasmas}

Thermal plasmas are high power discharges (from a few to hundred kWs) usually operated at or close to atmospheric pressures, and are demonstrated as effective environments for synthesizing Si-NPs using both $\mathrm{DC}^{[153-155]}$ and $\mathrm{RF}^{[156,157]}$ sources. Thermal plasmas have electron and gas temperatures exceeding well above $1 \mathrm{eV}$, enabling processing of nanomaterials with high melting points. Therefore, the 

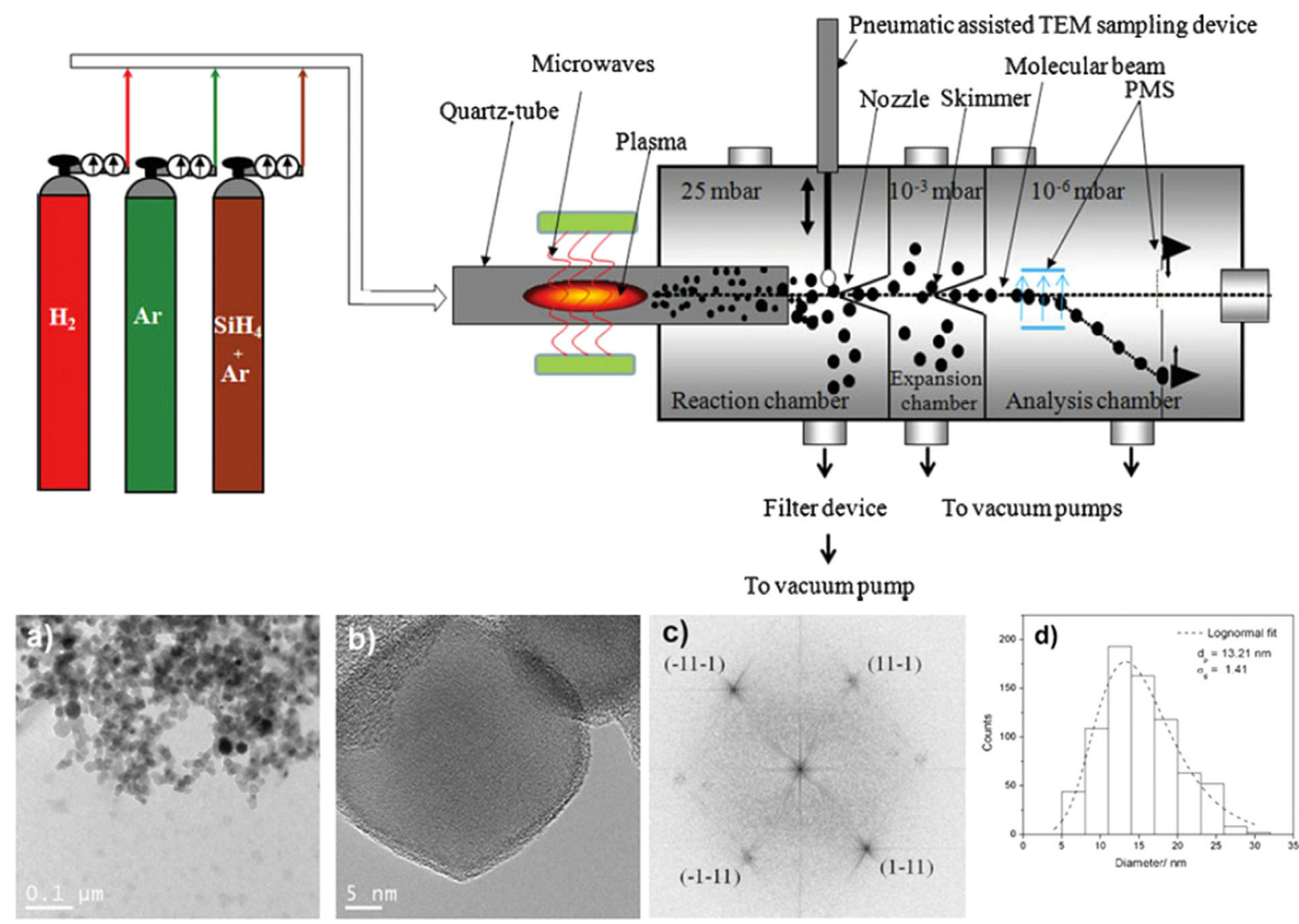

Figure 7. Schematic at the top demonstrates a microwave plasma, and images at the bottom demonstrates (a) TEM and (b) HRTEM images of SiNPs, (c) their fast Fourier transform (FFT) image, showing the crystalline morphology of synthesized Si-NPs, and (d) particle size distribution of SiNPs synthesized in the microwave plasma. (Reprinted with permission from Gupta et al. ${ }^{[68]}$ Copyright 2009, Wiley-VCH Verlag.)

precursor material can not only be in the gas form, but also in the form of micrometer-sized solid particles to be completely melted/evaporated and nano-processed (Figures 8 and 9). Whether the precursor is in the solid or in the gas form, their complete evaporation and decomposition ends up with formation of a supersaturated vapor of $\mathrm{Si}$ atoms, which then initiates growth by vapor-phase nucleation within the thermal plasma zone. Si-NPs grow further in the downstream by means of co-condensation and coagulation, where the gas temperature is less than $0.4 \mathrm{eV} \cdot{ }^{[155,157]}$ On the other hand, Girshick have reported that in the absence of supersaturated vapor, chemical nucleation plays the key role on the growth of nanoparticles. ${ }^{[158,159]}$ The group of Colombo have performed a series of studies on the modeling of the formation of Si-NPs in thermal plasmas. ${ }^{[156,160-162]}$ They investigated various mechanisms on Si-NP formation including fluid dynamics studies, turbulent effects on melting and evaporation of precursor, reactor geometry on the size of synthesized Si-NPs, all providing deeper insight on nanoparticle growth and formation in thermal plasmas.
Size control of Si-NPs is achieved via tuning of the residence time and substrate-to-nozzle distance, and in average, primary particle sizes of 5-20 nm with crystalline morphologies can be obtained. ${ }^{[153-155]}$ Coagulation results in the formation of Si-NPs in $200-300 \mathrm{~nm}$ range. ${ }^{[157]}$ The extreme high power, and the ability of feeding solid precursor through the thermal plasma zone results in very high throughputs of more than $300 \mathrm{~g}$ of Si-NPs per hour, which is among the highest Si-NP processing throughput reported using plasmas. ${ }^{[157]}$ Apart from Si-NPs, synthesis of various nanoparticles in thermal plasmas such as $\mathrm{SiO}_{2},{ }^{[163]}$ $\mathrm{SiO}_{\chi},{ }^{[164]} \mathrm{SiO}_{\chi}-\mathrm{Ti}^{[165]} \mathrm{SiC},{ }^{[166]} \mathrm{TaC}^{[167]} \mathrm{Al}_{2} \mathrm{O}_{3},{ }^{[168]} \mathrm{TiO}_{2},{ }^{[169]}$ and W-bronze ${ }^{[170]}$ have been reported.

\subsubsection{Remote Expanding Thermal Plasma}

Remote expanding thermal plasma (ETP) has been used for the deposition of the materials like hydrogenated amorphous silicon $(a-\mathrm{Si}: \mathrm{H}),{ }^{[171-173]}$ amorphous carbon $(a-\mathrm{C}: \mathrm{H}),{ }^{[174]}$ silicon dioxide $\left(\mathrm{SiO}_{2}\right),{ }^{[175]}$ silicon nitride $\left(a-\mathrm{SiN}_{\chi}: \mathrm{H}\right),{ }^{[176]}$ carbon nitride $(\mathrm{a}-\mathrm{C}: \mathrm{N}: \mathrm{H}),{ }^{[177]}$ and zinc oxide 
a)
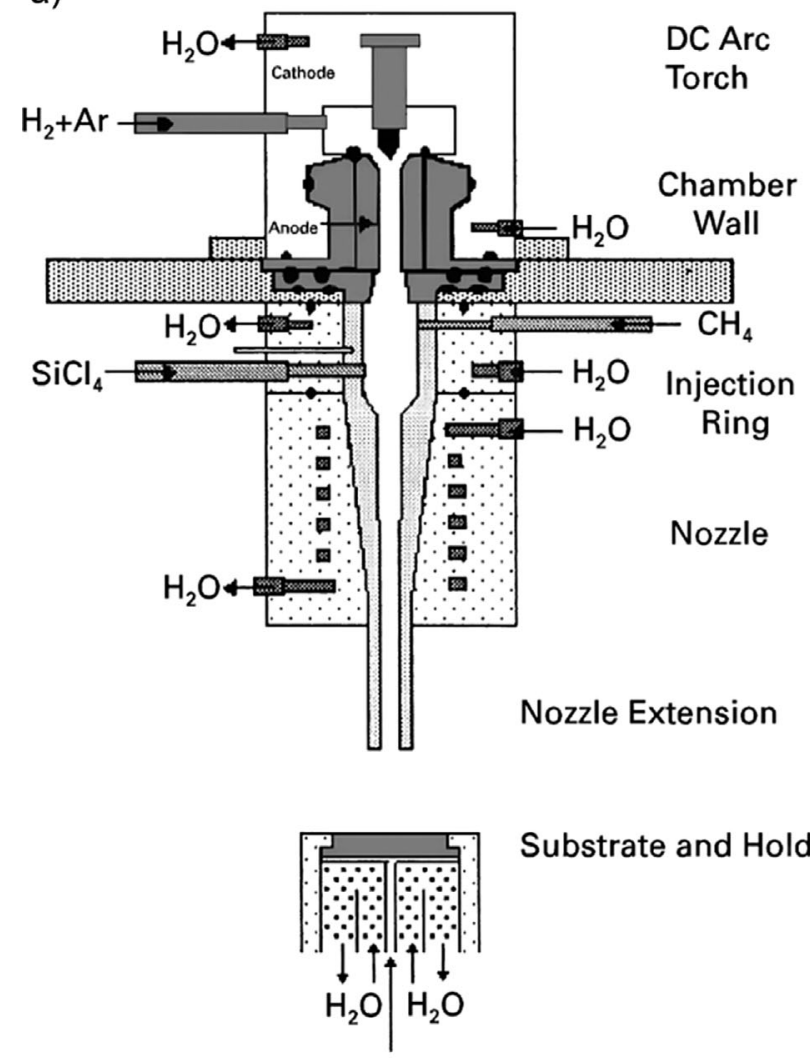

\section{Substrate and Holder}

b)

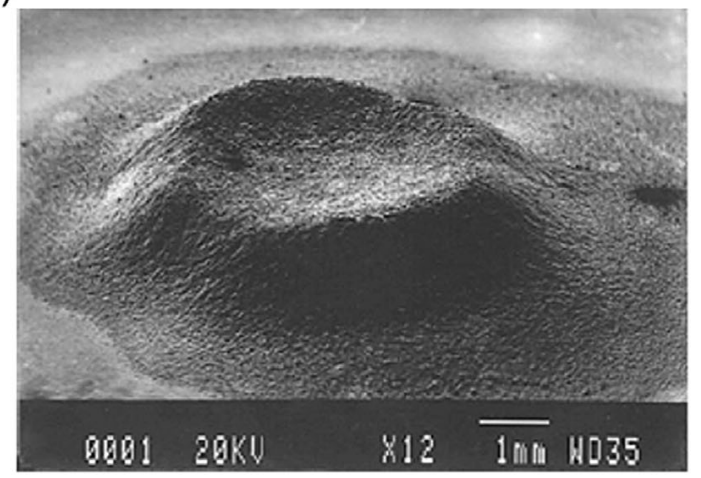

C)

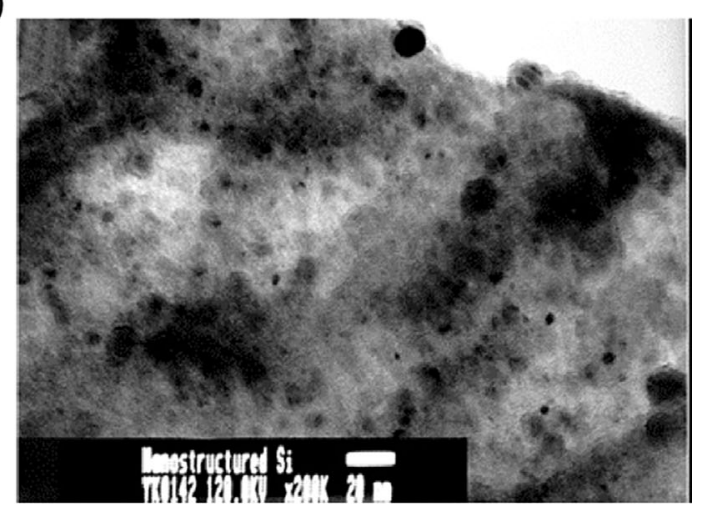

\section{Ar-He Mixture}

Figure 8. (a) Schematics demonstrating a hypersonic plasma deposition setup, a type of thermal plasma deposition tool; (b) SEM; and (c) TEM images of Si-NPs synthesized in the thermal hypersonic plasma expansion. (Reprinted with permission from Rao et al. ${ }^{[155]}$ Copyright 1998, Elsevier.)

(ZnO:Al). ${ }^{[178]}$ A major advantage of the ETP is its capability to establish high rate deposition of these materials with good film qualities.

The ETP is a remote plasma, i.e., the plasma production, transport, and deposition regions are geometrically separated (Figure 10). A background plasma (generally from $\mathrm{Ar}$ gas) is thermally generated in a cascaded arc plasma source applying DC current driven power $(1-5 \mathrm{~kW})$ under subatmospheric pressures (100-500 mbar) with electron and gas temperatures around $1 \mathrm{eV} \cdot{ }^{[179,180]}$ The cascaded arc is connected to a low pressure vessel $(0.2-2.0 \mathrm{mbar})$, where the plasma rapidly expands downstream -first supersonically until a stationary shock, and then subsonically $^{[181]}$-with electron temperatures in the range $0.1-0.3 \mathrm{eV}$ and ion temperatures well below $0.2 \mathrm{eV}$ (Figure 11). ${ }^{[180]}$ Unlike the other so-called remote plasmas -such as RF-PECVD -there is no electrode at any point in the downstream deposition chamber, including the substrate. In addition, the precursor is not injected in the thermal zone, i.e., the cascaded arc, but in the downstream deposition vessel, where the plasma has non-thermal characteristics. The remote feature of the ETP brings major advantages in material processing such as the absence of any downstream plasma originated effects on the plasma production zone, absence of a direct plasma contact with the substrate, low amount of high energy ion bombardment, and low UV light exposure on the deposited film. Since the properties of plasma production and deposition zones do not affect each other, independent variation of the process parameters in these zones enable better optimization of the synthesized material properties. This feature enables reaching high throughputs without a loss in the quality of the processed material.

In ETP, material synthesis is realized by the reactions between the molecules, ions, and radicals formed after the dissociation of the injected molecular gas. In previous studies, our group have observed the formation of small cationic Si clusters up to ten $\mathrm{Si}$ atoms during the processing of microcrystalline Si films. ${ }^{[182,183]}$ Observation of cationic Si clusters is expected in the ETP downstream: the dissociation of $\mathrm{SiH}_{4}$ is realized by $\mathrm{Ar}^{+}$ions as the electrons do not have enough energy to dissociate $\mathrm{SiH}_{4}$ molecules. This is a unique feature of the ETP with respect to other plasmas, where the electron-induced chemistry is 

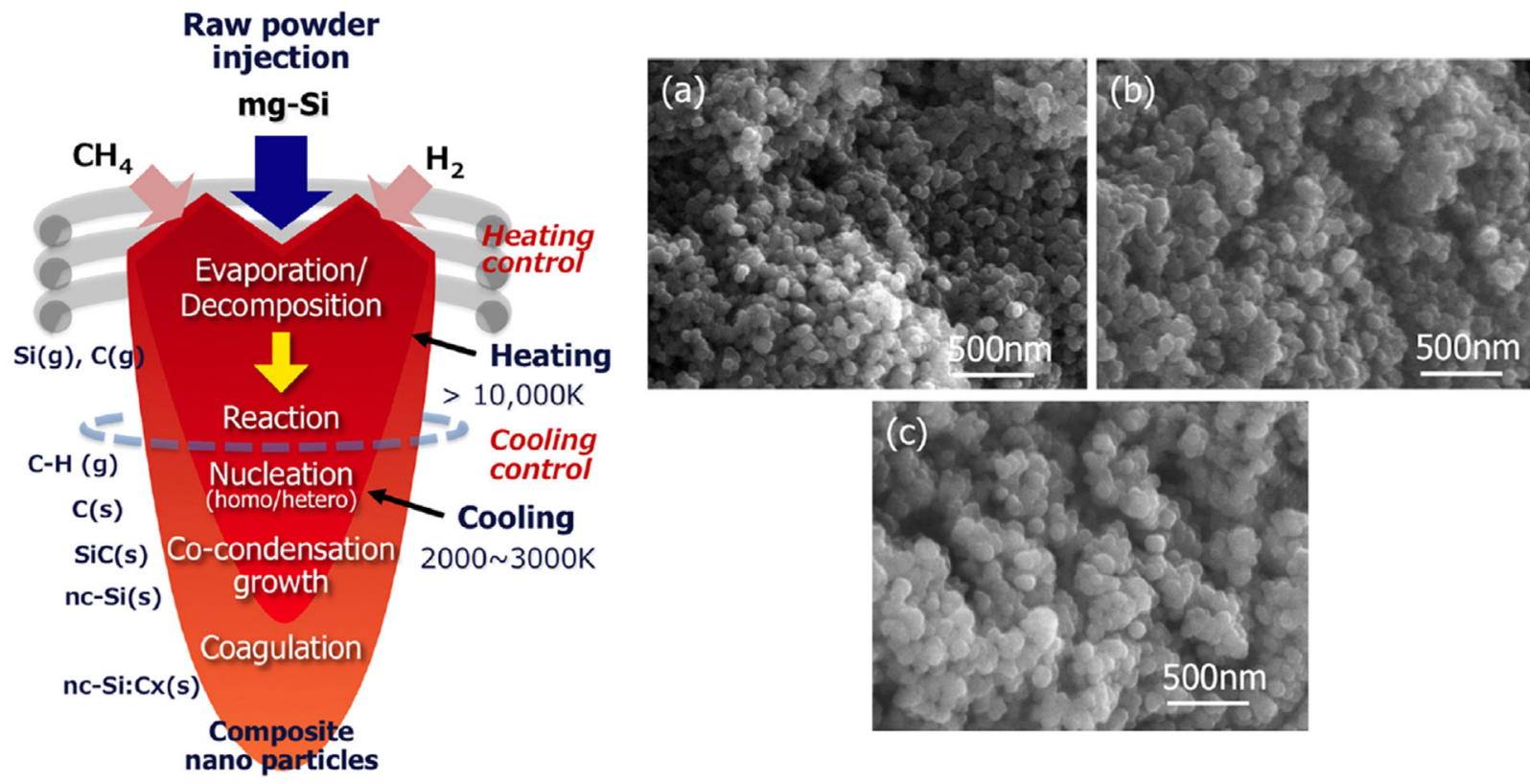

Figure 9. Plasma spray physical vapor deposition is shown at the left, a type of thermal plasma, in which metallurgical grade silicon ( $m g-S i)$ is used as the injected material. At the right, SEM images of plasma sprayed nanocomposite C/Si powders at C/Si molar ratios of (a) 1.0, (b) 1.5, and (c) 3.0. $\mathrm{mg}^{-\mathrm{Si}}$ is injected with a rate of $1 \mathrm{~g} \mathrm{~min}^{-1}$. (Reprinted with permission from Kambara et al. ${ }^{[157]}$ Copyright 2014, AIP Publishing LLC.)

dominant. Therefore, in the ETP, the mechanisms that lead to the growth of Si-NPs are different. Si-NP growth starts via dissociation of $\mathrm{SiH}_{4}$ molecules with $\mathrm{Ar}^{+}$ions in a dissociative charge-exchange reaction that results in the formation of $\mathrm{SiH}_{m}{ }^{+}$ions. ${ }^{[46,47,182,184]}$ The formed molecular ions can subsequently be involved in two different polymerization pathways, i.e., radical polymerization and ion polymerization. In the first pathway, $\mathrm{SiH}_{m}{ }^{+}$ions react with electrons via dissociative recombination reactions forming $\mathrm{SiH}_{m}$ radicals. ${ }^{[46]}$ Radical polymerization continues by addition of $\mathrm{SiH}_{4}$ molecules via association reactions leading to formation of $\mathrm{Si}_{n} \mathrm{H}_{m}$ radicals. ${ }^{[185]}$ In the second pathway $\mathrm{SiH}_{m}{ }^{+}$ions react with $\mathrm{SiH}_{4}$ molecules via associative charge-exchange reactions forming $\mathrm{Si}_{n} \mathrm{H}_{m}{ }^{+}$ions. ${ }^{[185]}$ In both of the pathways, further growth occurs via addition of the reactive species in the plasma leading to the formation of stable and fully crystalline Si-NPs. During the nucleation, the growth reactions and atomic hydrogen association

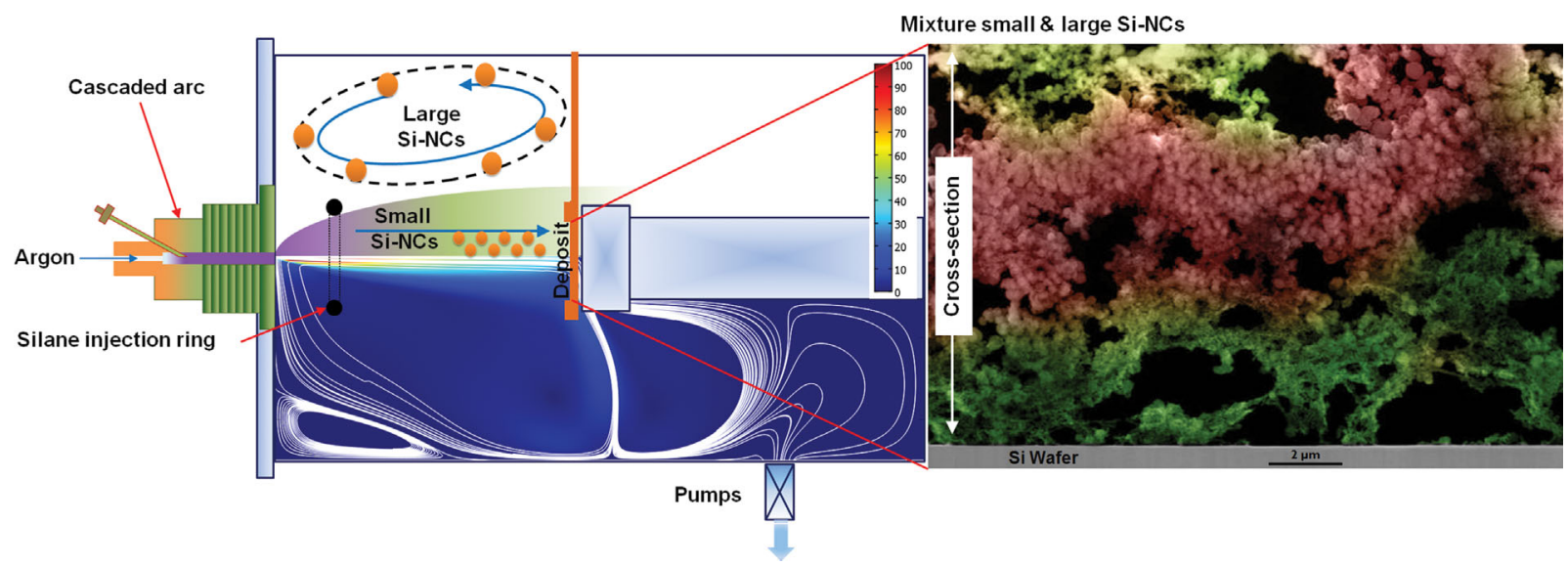

Figure 10. A remote expanding thermal plasma setup used to synthesize free-standing crystalline Si-NPs. At the bottom half of the plasma deposition tool, the overall 2D flow pattern is demonstrated. At the top half, formation regions of small and large Si-NPs are shown. A falsecolored cross-sectional TEM image of Si-NPs demonstrate the variations in size and the voids in the deposited powder. (Reprinted with permission from Doğan et al. ${ }^{[48]}$ Copyright 2015, IOP Publishing. All rights reserved.) 


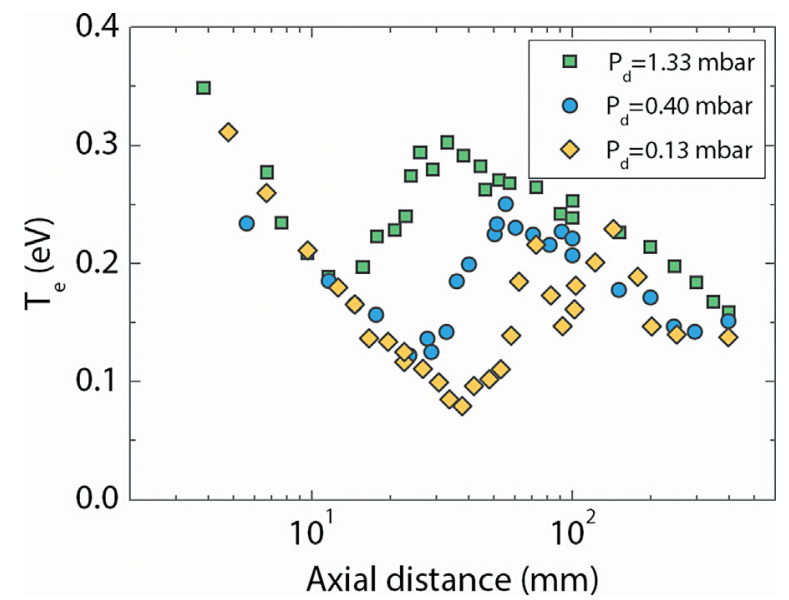

Figure 11. Electron temperature in the downstream of the ETP as a function of background pressures from 0.13 to $1.33 \mathrm{mbar}$. (Reprinted with permission from van de Sanden et al. ${ }^{[180]}$ Copyright 1993, American Physical Society.)

reactions occurring on the surface of the nanoparticle sustain crystallinity of Si-NPs as a result of the energy released from these chemical reactions. ${ }^{[47,186]}$ In a controlled study of time modulated $\mathrm{SiH}_{4}$ injection in the millisecond range, we have observed that the increase in the local density of $\mathrm{SiH}_{4}$ does not increase the size of Si-NPs produced, but it rather increases the number density of Si-NPs and reduces the average size of these crystalline nanoparticles. We have explained this phenomena in terms of rapid depletion of plasma species at higher number density of primary particles (as a result of increased available surface area), leading to limited growth of Si-NPs. In addition, the rapidly cooling gas temperature prevents nanoparticle growth via sintering/coalescence and forms soft agglomerates. These observations revealed that the growth mechanism of Si-NPs in the ETP is dominated by nucleation and CVD growth on the nanoparticle surface, and coagulation is highly unlikely. ${ }^{[4]}$

Si-NP sizes were determined by the residence time distributions in the downstream plasma, which leads to a bimodal Si-NP size distribution ${ }^{[46,187]}$ as a result of a rapidly expanding central beam (residence times of less than $10 \mathrm{~ms}$ ), and surrounding background recirculation cells (residence times of 0.1-0.4 s), see Figure $10 .{ }^{[48]}$ Si-NPs synthesized in the central beam are in the range $2-10 \mathrm{~nm}$, and those that are synthesized in the recirculation cells are in the range $50-120 \mathrm{~nm}$ (Figures 12 and 13). According to the ion-flux measurements that we have performed in the downstream of the ETP, small Si-NPs were formed in the central beam mainly via ion-driven nucleation pathway (mainly cations are involved), and large Si-NPs are formed mainly via radical-driven nucleation pathway in the recirculation cells. We further verified this observation by increasing the volume fraction of synthesized Si-NPs with an increase of the ion density in the plasma. ${ }^{[4]}$ The high processing rate of Si-NPs $\left(100 \mathrm{mg} \mathrm{min}^{-1}\right)$ and scalability of the reactor dimensions with controlled material properties makes ETP an attractive tool for industrial scale applications.

\subsection{Functionalization}

Functionalization of Si-NP surfaces is essential for controlling the surface-related effects as the state of the surface plays a critical role on the optical, electrical, and chemical properties. One of the aims for performing functionalization is to passivate surface defects that diminish the size-dependent features of Si-NPs. Another aim of functionalization is to bring additional features, which otherwise do
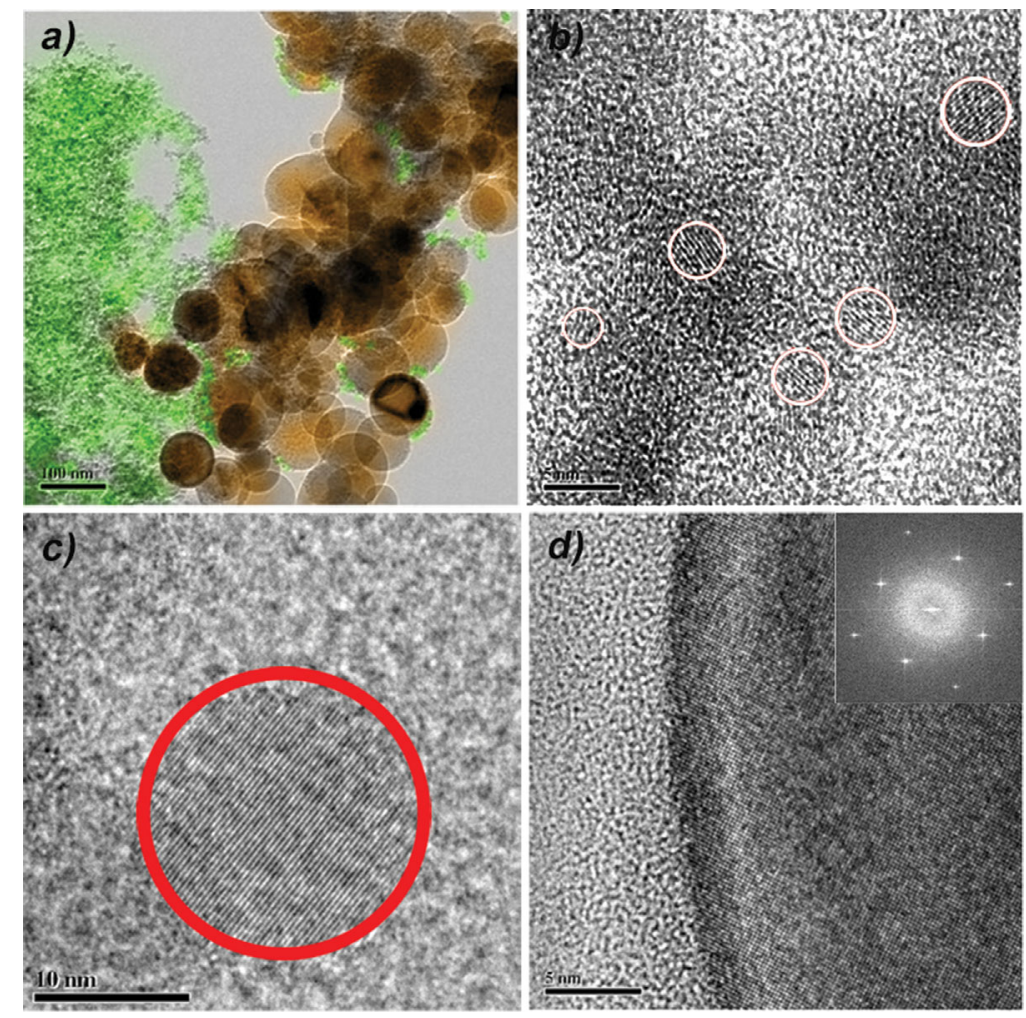

Figure 12. (a) False-colored TEM images of Si-NPs with a bimodal size distribution, (b) small Si-NPs with sizes of $4 \mathrm{~nm}$, (c) $10 \mathrm{~nm}$, and (d) a large Si-NP with a size of $80 \mathrm{~nm}$ are demonstrated using HRTEM. FFT image at (d) demonstrates the crystalline morphology. (Reprinted with permission from Doğan et al. ${ }^{[46]}$ Copyright 2013, AIP Publishing LLC.) 


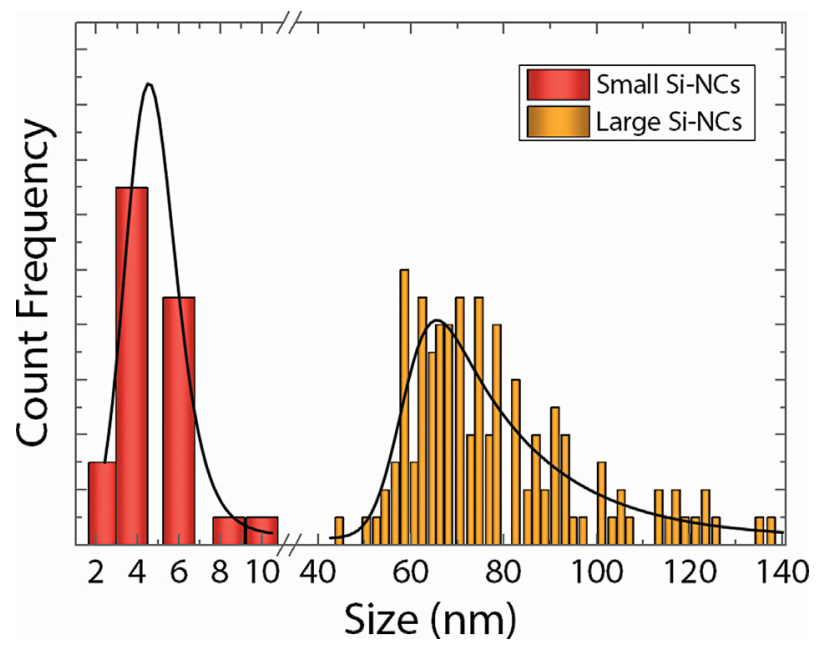

Figure 13. Statistical size distribution of Si-NPs estimated from TEM images. The presence of a bimodal size distribution of Si-NPs in the range $2-10 \mathrm{~nm}$ to $50-120 \mathrm{~nm}$ is clearly seen. (Reprinted with permission from Doğan et al. ${ }^{[46]}$ Copyright 2013, AIP Publishing LLC.)

not exist in Si-NPs, or to improve the existing size-dependent properties. Functionalization can also enable excellent dispersion of Si-NPs in water ${ }^{[188,189]}$ or in organic solvents, ${ }^{[190]}$ which brings the advantage of improved biocompatibility and controlled manipulation of Si-NPs for device-level applications. Most of the times, a successful functionalization step establishes all these aims. For instance, hydrosilylation routes of Si-NPs passivates the surface defects, improves stability of Si-NPs against oxidation via establishing a high-degree of surface coverage, and improves the optical properties and significantly reduces the mitigation of these properties in time. ${ }^{[49,191]}$ The most suitable environment in order to perform such kind of functionalization routes should ideally establish the synthesis and functionalization in the same batch, i.e., without exposing Si-NPs against uncontrolled reactive media like air. In this context, plasmas are very suitable environments for both synthesis and in situ functionalization of Si-NPs at the same time. Here, we will highlight some case studies of in situ functionalization of Si-NPs in plasmas during/after gasphase synthesis.

One of the mostly observed functionalization process of Si-NPs in plasmas occurs via the coverage of nanoparticle surfaces by the dissociated atoms of the Si-containing precursor during the synthesis. It was reported that using precursors of $\mathrm{SiH}_{4},{ }^{[117,192]}$ and $\mathrm{SiCl}_{4}{ }^{[40,117,193]}$ leads to the formation of Si-NPs covered with hydrogen and chlorine, respectively. The research demonstrated that hydrogen terminated Si-NPs are significantly more robust against oxidation in air than chlorine terminated Si-NP surfaces, which oxidizes almost instantly. ${ }^{[117]}$

One of the first all-gas-phase approach of Si-NP functionalization was demonstrated by the group of
Kortshagen. ${ }^{[36,37,194]}$ Si-NPs were synthesized using a non-thermal plasma reactor, which is connected to another non-thermal capacitively coupled plasma reactor, see Figure 14. Synthesized Si-NPs were directly transported into the second plasma, where the vapor of various liquid organic precursors were also injected with Ar as the carrier gas. In this particular example, decomposition of 1-dodecene in the second plasma resulted in grafted Si-NP surfaces. Functionalized Si-NPs demonstrated excellent solubility in liquids (in this case toluene) forming stable nanoparticle inks, and more importantly demonstrated high photoluminescence quantum yields exceeding $60 \%$.

The group of Agarwal have also demonstrated in situ gasphase hydrosilylation of Si-NPs synthesized in a nonthermal plasma similar to the reactor design of the group of Kortshagen. Jariwala et al. ${ }^{[49]}$ used short-chain alkynes and long-chain alkenes by means of in situ gas-phase hydrosilylation to reach an improved surface coverage of Si-NPs and stable optical properties against oxidation in air. They first have observed surface coverages of 38 and 30\% using styrene and acetylene hydrosilylation, respectively. They then demonstrated that by selecting proper sequence of short-chain alkyne followed by long-chain alkene

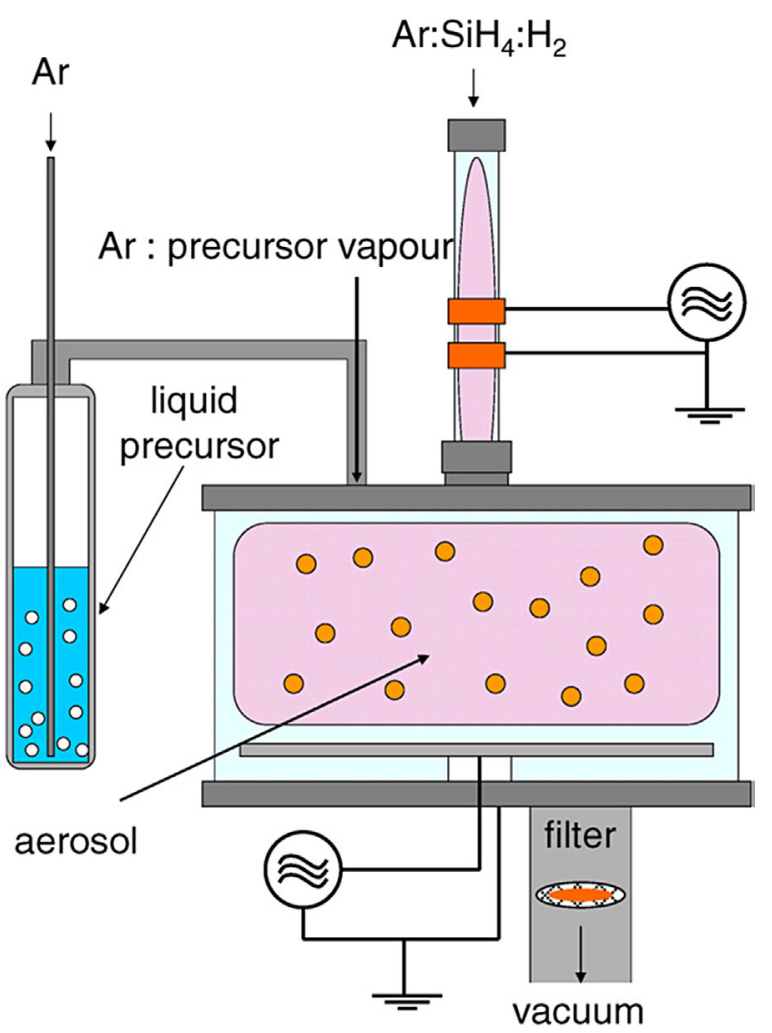

Figure 14. Schematics of the non-thermal plasma system for the synthesis and in situ functionalization of Si-NPs. (Reprinted with permission from Mangolini et al. ${ }^{[36]}$ Copyright 2007, Wiley-VCH Verlag.) 
hydrosilylation, $50 \%$ surface coverage of Si-NPs could be achieved (Figure 15). In a follow up study, Weeks et al. ${ }^{[50]}$ reported a surface coverage of $58 \%$, which is close to the theoretical maximum of $60 \%$ for alkenyl terminated $\mathrm{Si}(111) \cdot{ }^{[49]}$ Improved surface coverage had a positive impact on the photoluminescence stability under ambient conditions, which is directly related to the resistance to oxidation in air, indicating that plasma-assisted functionalization routes can actually be used to preserve/improve the size-dependent features of Si-NPs.

Another example of surface functionalization of Si-NPs is demonstrated using a double plasma, where Si-NP synthesis and the plasma decomposition of functionalizing agents takes place simultaneously in different plasmas, and co-mixed in the downstream. The group of Shiratani used double multi-hollow capacitive high frequency discharge to synthesize surface nitridated Si-NPs. ${ }^{[51,52]}$ In their particular setup Si-NPs were synthesized in $\mathrm{SiH}_{4} / \mathrm{H}_{2}$ gas mixture in one discharge, and a nitrogen plasma is generated in the
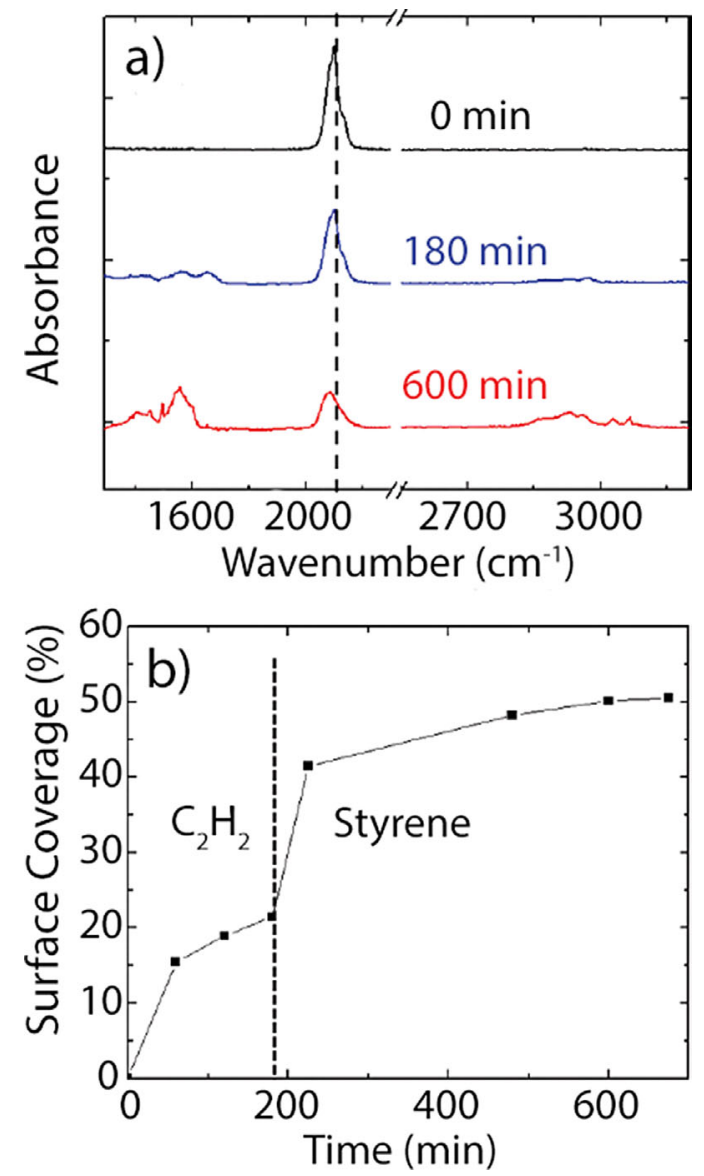

Figure 15. (a) IR absorbance spectrum of Si-NPs. As-synthesized Si-NPs (o min) were first exposed to $\mathrm{C}_{2} \mathrm{H}_{2}$ for $180 \mathrm{omin}$, and then to styrene for $420 \mathrm{~min}$ (in total, $600 \mathrm{~min}$ ) at $160^{\circ} \mathrm{C}$. (b) Evolution of surface coverage of Si-NPs as a function of time during sequential acetylene and styrene exposure. (Reprinted with permission from Jariwala et al. ${ }^{[49]}$ Copyright 2011, American Chemical Society.)

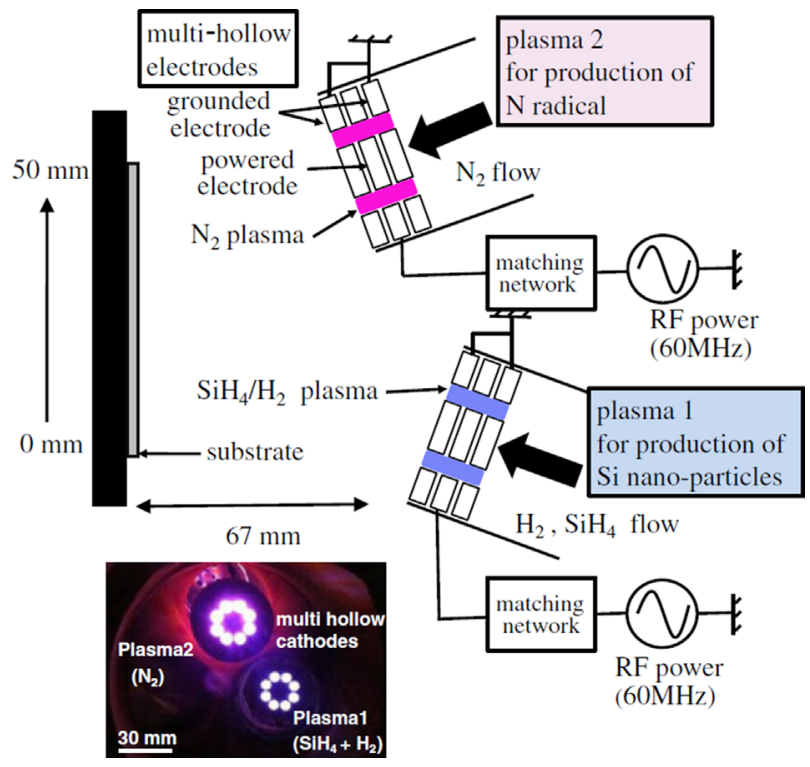

Figure 16. Schematics demonstrating the double multi-hollow capacitive high frequency discharge used to synthesize nitridated Si-NPs in a one-step plasma processing route. (Reprinted with permission from Uchida et al. ${ }^{[52]}$ Copyright 2012, The Japan Society of Applied Physics.)

other discharge (Figure 16). They demonstrated that this one-step double plasma process improves the incident photon-to-current conversion efficiency (IPCE) of Si-NPs when embedded in solar cells from 20 to $40 \%$-especially in the UV range from 350 to $400 \mathrm{~nm}$-when compared to Si-NPs without nitridation. ${ }^{[51,52]}$

Apart from the in situ plasma based functionalization, easy manipulation of gas-phase plasma synthesized Si-NPs enables post-functionalization routes. We will not deal with these processes, however we should note some of the important examples here. These examples involve liquidphase hydrosilylation of Si-NPs with various alkenes ${ }^{[65,66,68,195]}$ and alkene/benzenes, ${ }^{[196,197]}$ hydrogen termination following HF etching, ${ }^{[62]}$ hydroxyl and organic ligand based functionalization in plasma electrochemical processes ${ }^{[149]}$ and silanization after HF/HNO etching. ${ }^{[198]}$

\section{Use of Si-NPs in Future Energy Applications}

\subsection{Solar Cells}

In solar cells, electricity is generated by means of conversion of photons to electron-hole pairs in the electrically active region, where these photo-generated carriers are subsequently separated by the internal field of a $p-n$ junction. The threshold absorption energy of a solar cell critically depends on the bandgap energy of the absorbing material used. According to the theoretical calculations for a 
bandgap energy of $1.12 \mathrm{eV}$ (bandgap energy of bulk $c$-Si), a single junction solar cell can reach a maximum efficiency of $31 \%$, which is known as the Shockley-Oueisser limit. ${ }^{\text {[199] }}$ This maximum efficiency barrier is strongly related with the fact that not all the photons are absorbed with the same efficiency. Consider a light absorbing material with a specific bandgap energy: the most efficient photon absorption takes place only at the photon energies that are matching with the bandgap energy of the absorber material. The photons lower than the energy of the bandgap are not absorbed, and the photons higher than the bandgap energy of the absorber layer will get partially thermalized at the expense of their excess energies above the bandgap energy. To overcome this limit, next generation solar cell concepts are proposed. ${ }^{[25,200-202]}$ One of the proposed solutions uses multiple stacks of layers with different bandgap energies as the absorbing part of the solar cell, which can efficiently absorb the light from different wavelengths of the solar spectrum. Another proposed solution is based on the advanced photon management concepts, where high energy photons are utilized before being absorbed by the active layer to reduce the thermal losses in the absorbing layer (down-shifting), or to create multiple lower energy photons (down-conversion, via MEG and SSOC process) that matches the bandgap energy of the absorbing layer.

Due to their size-dependent bandgap energy, Si-NPs can act as the active material in the multi-layer solar cell approach, absorbing photons, generating electron-hole pairs, and directly contributing to the photocurrent. Multi-layer solar cells based on Si-NPs can potentially promote higher efficiencies by covering a wider range of the solar spectrum with respect to a single junction solar cell. ${ }^{[25]}$ In this concept, the structural arrangement of Si-NP multilayers should be adjusted in such a way that the bandgap energy should decrease-or in other words, the size of Si-NPs in the sequential layers should increase-from top to the bottom of the active region to achieve minimal loss during the absorption of photons with different energies, see Scheme 3. In addition, Si-NPs can be one of the active layers of a multi-junction solar cell, where the other junctions are composed of various materials with different bandgap energies. Theoretical calculations demonstrate that efficiencies up to $42.5-47.5 \%$ can be realized using two and three junction tandem solar cells. ${ }^{[203,204]}$

As we mentioned before, Si-NPs can also be employed in the concept of advanced photon management by means of the down-shifting, ${ }^{[23,196,205-207]}$ and down-conversion

\section{Silicon Nanoparticles in Solar Cells}

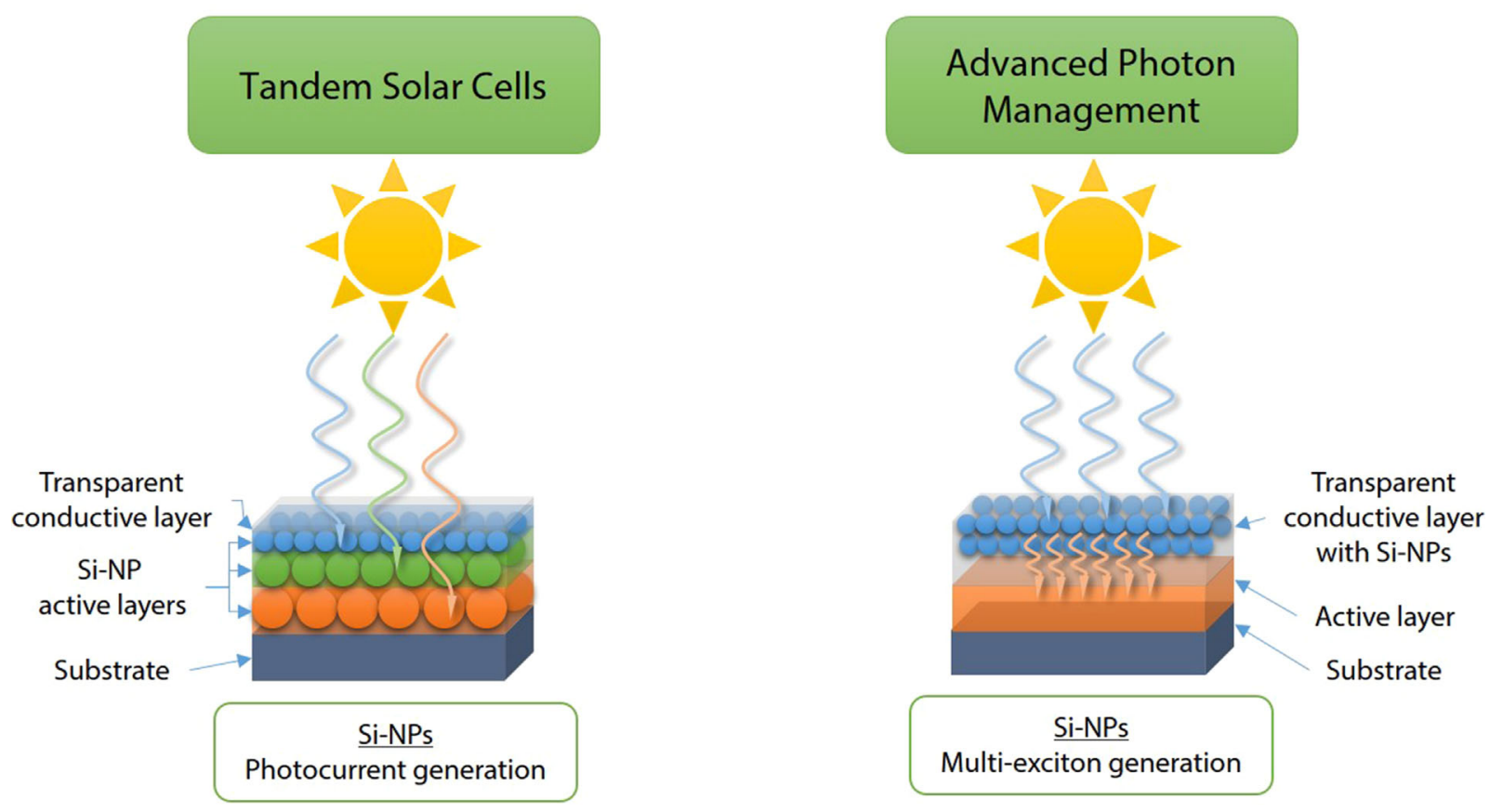

Scheme 3. Si-NPs in solar cell concepts. Use of Si-NPs in solar cell applications can be done in two ways. The first way is to embed Si-NPs in multi-layer solar cells as the active light absorbing material, generating photocurrent by capturing light from the various regions of solar spectrum. The second way is in the concept of advanced photon management, where Si-NPs act as down-shifter or down-converter layers on top of the finished solar cells, reducing thermal losses in the active region, or improving the utilization of hot photons by creating multiple low energy photons, respectively. 
process via MEG and SSOC. ${ }^{[57,71-75,80]}$ In this concept, Si-NPs are not in electrical contact with the active region of the solar cell. Instead, Si-NPs are put in a transparent conductive layer on top of the solar cell, which act as a down-shifter/converter, see Scheme 3. Particularly for the down-conversion process to occur, photons at least two times of the bandgap energy of the absorbing layer are required. For example, a down-converter material on top of a $c$-Si solar cell with a bandgap energy of $\sim 1.1 \mathrm{eV}$ should be able to absorb the photons with a minimum energy of $2.2 \mathrm{eV}$ and create two photons with energies $\sim 1.1 \mathrm{eV}$ via MEG or SSOC. In this sense, size-controlled Si-NPs could be efficient down-converters, considering their sizedependent bandgap energies, extending to the high energy part of the solar spectrum up to $\sim 5 \mathrm{eV} .{ }^{[59]}$

Attempts on realizing improved efficiency solar cell devices using gas-phase plasma synthesized Si-NPs led to encouraging results. A series of studies from the group of Shiratani focused on both fundamental and practical aspects of Si-NP properties and the solar cells produced using Si-NPs synthesized in (multi-hollow) capacitive high frequency discharges (Figure 16). In a study focusing on the effect of size dispersion of Si-NP films, they observed a dramatic reduction of the electron mobility of up to $50 \%$ if there is a dispersed size distribution of Si-NPs in the film, even if all the sizes are in the confinement region. ${ }^{[4]}$ In another study, Kawashima et al. ${ }^{[208]}$ demonstrated the functionality of $\mathrm{Si}$ quantum dot-sensitized solar cells, where Si-NPs were mixed with $\mathrm{TiO}_{2}$ paste as the sensitizer. In this concept, Si-NPs with sizes of $17.2 \mathrm{~nm}$ were used in order to have the lowest possible bandgap for facilitating carrier multiplication by absorbing the high energy photons. Solar cells sensitized with Si-NPs demonstrated 2.5\% incident photon-to-current conversion efficiency (IPCE). In contrast, solar cells without Si-NPs demonstrated IPCE of $1.5 \%$. The increase in the IPCE was attributed to the carrier generation in Si-NPs, mainly taking part in the wavelength region below $500 \mathrm{~nm}$.

The group of Shiratani have also used Si-NPs to further increase the efficiency of Ru dye-sensitized solar cells in the concept of hybrid-sensitized solar cells. ${ }^{[209]}$ Using Si-NPs they have improved the overall efficiency of Ru dyesensitized solar cells from 2.4 to $3.0 \%$, and the power conversion efficiency from 20 to almost 25\% (Figure 17). They observed that by increasing the Si-NP/TiO 2 weight ratio, both the power conversion efficiency and the short circuit current increase. The authors commented on two mechanisms that Si-NPs contribute to the enhanced efficiency. First, Si-NPs improve the carrier generation as a result of their contribution to the increased absorption especially in the UV range (200-500 nm); second, increased effective surface area of Si-NPs for Ru dye attachment.

Functionalization and passivation of Si-NPs and their impact on the solar cell properties were also investigated by the same group. Hoo et al. ${ }^{[210,211]}$ used functionalized Si-NPs (with 4-vinylbenzoic acid) with sizes of $17 \mathrm{~nm}$ and $\mathrm{ZnO}$ barriers in quantum dot-sensitized solar cells to improve the charge carrier generation, and prevent the electron recombination at the electrolyte, respectively. Si-NPs improved the efficiency of fluorine based tin oxide/ titanium dioxide solar cells from a mere 0.002 to $0.012 \%$. Functionalizing the Si-NP surfaces further improved the overall efficiency to $0.036 \%$ and using ZnO barriers finally resulted in efficiencies about $0.045 \%$. It was also observed that the IPCE is improved about $11 \%$ in the range $300-450 \mathrm{~nm}$ upon functionalizing Si-NPs. Uchida et al. ${ }^{[212]}$ observed that passivation of Si-NP surfaces upon exposure to $\mathrm{CH}_{4}$ or $\mathrm{N}_{2}$ plasma improved the photocurrent generation in Si-NP sensitized solar cells. They observed that the short circuit current increased by a factor of 2.5 , which they related this increase with the surface passivation. Finally, absorbed photon-to-current conversion efficiency at $300 \mathrm{~nm}$ was observed as $60 \%$.

The Shiratani group have also studied the effect of adsorbance of Si-NPs to nano-porous $\mathrm{TiO}_{2}$ on the performance of paint-type Si-NP sensitized $\mathrm{TiO}_{2}$-based solar cells. ${ }^{[213,214]}$ They reported that low level of adsorbance of Si-NPs to $\mathrm{TiO}_{2}$ is a cause of low performance. Mixing Si-NPs and $\mathrm{TiO}_{2}$ and sintering them together at $200^{\circ} \mathrm{C}$ and repeating this procedure for a multiple times to improve the absorption of light resulted in higher photocurrent densities and better performances. The highest observed efficiency from this study was reported as $0.14 \%$.

In their recent studies, higher efficiencies of Si-NP based solar cells have been reached. In a work on the characteristics of organic/inorganic hybrid solar cells, Uchida 
et al. ${ }^{[216]}$ used Si-NPs in poly(3,4-ethylenedioxythiophene): poly(styrene sulfonate) (PEDOT:PSS)/c-Si solar cells as the current generators. For the active Si-NP layer with a thickness of $110 \mathrm{~nm}$, they analyzed the energy conversion efficiency of these hybrid solar cells and obtained a maximum efficiency of $2.84 \%$ and a high IPCE of $82.8 \%$ at $460 \mathrm{~nm}$ as a result of improved carrier generation from Si-NP interfaces. Seo et al. ${ }^{[215]}$ reported on the efficiency of Si-NP and dye co-sensitized solar cells. Although the efficiency of Si-NP based solar cell was $0.035 \%$ and the efficiency of dye (N749) sensitized solar cell was 4.358\%, their co-sensitization yielded an overall enhanced efficiency of 5.1\%. In addition, the contribution of Si-NPs to the short circuit current was more than $1 \mathrm{~mA} \mathrm{~cm}^{-2}$, with a total short circuit current of about $10.2 \mathrm{~mA} \mathrm{~cm}^{-2}$ for the cosensitized cell, see Figure 18 . The success of using Si-NPs as a co-sensitizers in dye-sensitized solar cells is based on the fact that Si-NPs are stable with the iodine/triiodided $\left(I^{-} / I_{3}{ }^{-}\right)$ electrolyte used for dye-sensitized solar cells, where other nanomaterials like $\mathrm{CdS}, \mathrm{CdSe}, \mathrm{PbS}$, and $\mathrm{PbSe}$ were shown to be unstable because of the corrosive effects of iodine on these materials. ${ }^{\text {[217-219] }}$

The group of Kortshagen used Si-NPs synthesized in nonthermal plasmas (Figure 3) to investigate the efficiency contribution of these nanoparticles in solar cells. They have reported on hybrid solar cells based on a blend of Si-NPs and poly-3(hexylthiophene) (P3HT), see Figure 19. ${ }^{[37,220]}$ In this hybrid solar cell, Si-NPs acted as a percolation network for electron conduction, and P3HT polymer was responsible for the conduction of holes. Authors demonstrated that the power conversion efficiency, open circuit voltage and the short circuit current depends on the size of Si-NPs as well as the Si-NP/P3HT volume ratio. Particularly, they observed that reducing the Si-NP size from $10-20 \mathrm{~nm}$ to $3-5 \mathrm{~nm}$ increased both the open circuit voltage and short circuit

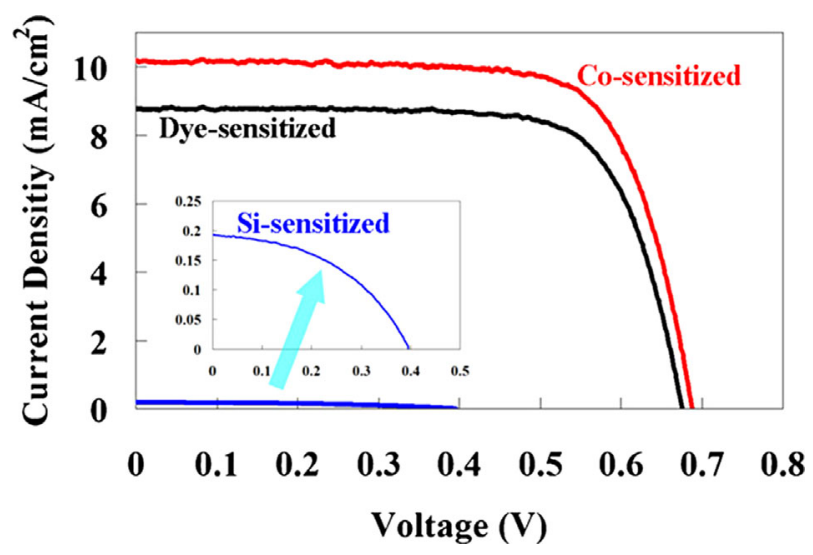

Figure 18. J-V characteristics of Si-NP sensitized solar cells, dyesensitized solar cells, and co-sensitized solar cells. (Reprinted with permission from Seo et al. ${ }^{[215]}$ Copyright 2015, The Japan Society of Applied Physics.) current. This is due to the opening of the bandgap of Si-NPs, which reduces the conduction band offset between the Si-NPs and P3HT, and reduces the voltage loss during exciton dissociation. Maximum efficiency was achieved with 35\% Si-NPs in Si-NP/P3HT blend, and with Si-NP sizes of 3-5 nm (Figure 20). Under A.M. 1.5 direct illumination $\left(100 \mathrm{~mW} \mathrm{~cm}^{-2}\right)$, this solar cell had a power conversion efficiency of $1.15 \%$, a short circuit current of $I_{\mathrm{sc}}=3.3 \mathrm{~mA}$ $\mathrm{cm}^{-2}$, and an open circuit voltage of $V_{\mathrm{oc}}=0.75 \mathrm{~V}$. The IPCE was found about $26 \%$ near $500 \mathrm{~nm}$.

Silicon nanoparticles were also demonstrated to improve the dark conductivity when they are incorporated into $a$-Si: $\mathrm{H}$ films. This was demonstrated by the group of Kortshagen, ${ }^{[222]}$ in a so-called dual-plasma environment. Si-NPs were produced using a non-thermal plasma with an average size of $5 \mathrm{~nm}$. The non-thermal plasma unit is connected to a downstream capacitively coupled discharge plasma, where in-flight transfer of Si-NPs and $a-\mathrm{Si}: \mathrm{H}$ deposition takes place simultaneously. When the fraction of Si-NPs is $\sim 0.02-0.04$ in the composite film, the dark conductivity was increased by several orders of magnitude with respect to the pure $a$-Si:H films, which are intended for solar cell applications. The increase in the dark conductivity was discussed in terms of charge contribution into the $a$-Si: $\mathrm{H}$ matrix via inclusion of Si-NPs.

In another report of Kortshagen's group, Liptak et al. ${ }^{[221]}$ investigated the environmental photostability of $\mathrm{SF}_{6}-$ etched Si-NPs during non-thermal plasma synthesis. They have observed that the optical properties of etched Si-NPs were improved upon exposure to air, unlike the hydrogen terminated and organically functionalized Si-NPs. PL quantum yield of Si-NPs were observed as 50\% (Figure 21). Moreover, the optical properties of Si-NPs were found to be stable against oxidation over the time of 3 years, against UV light exposure, and thermal treatment up to $160^{\circ} \mathrm{C}$. With these features, authors commented that $\mathrm{SF}_{6}$-etched Si-NPs are potential candidates as stable down-shifters to enhance the performance of solar cells.

The group of Pi and Yang have published two reports demonstrating the light absorption and down-shifting related improvement of non-thermal plasma synthesized Si-NPs on top of the solar cells. In their first report Pi et al. ${ }^{[23]}$ used porous Si-NP films spin-coated on screen-printed $\mathrm{Si}$ solar cells and demonstrated an absolute efficiency enhancement from 16.9 to $17.5 \%$ (the efficiency enhancement of Si-NP films were about 3.7\%). In this work, Si-NPs were optically coupled to the solar cell without any electrical contact. One hundred and twenty nanometerthick porous films of Si-NPs demonstrated $\sim 2.3 \%$ increase in the short circuit current, which was related to the increased absorption of the sunlight. This was accompanied by the significant reduction of the solar cell reflectance by the porous Si-NP film. The reflection reduction in the spectral range $300-1000 \mathrm{~nm}$ both observed to be dependent on the 


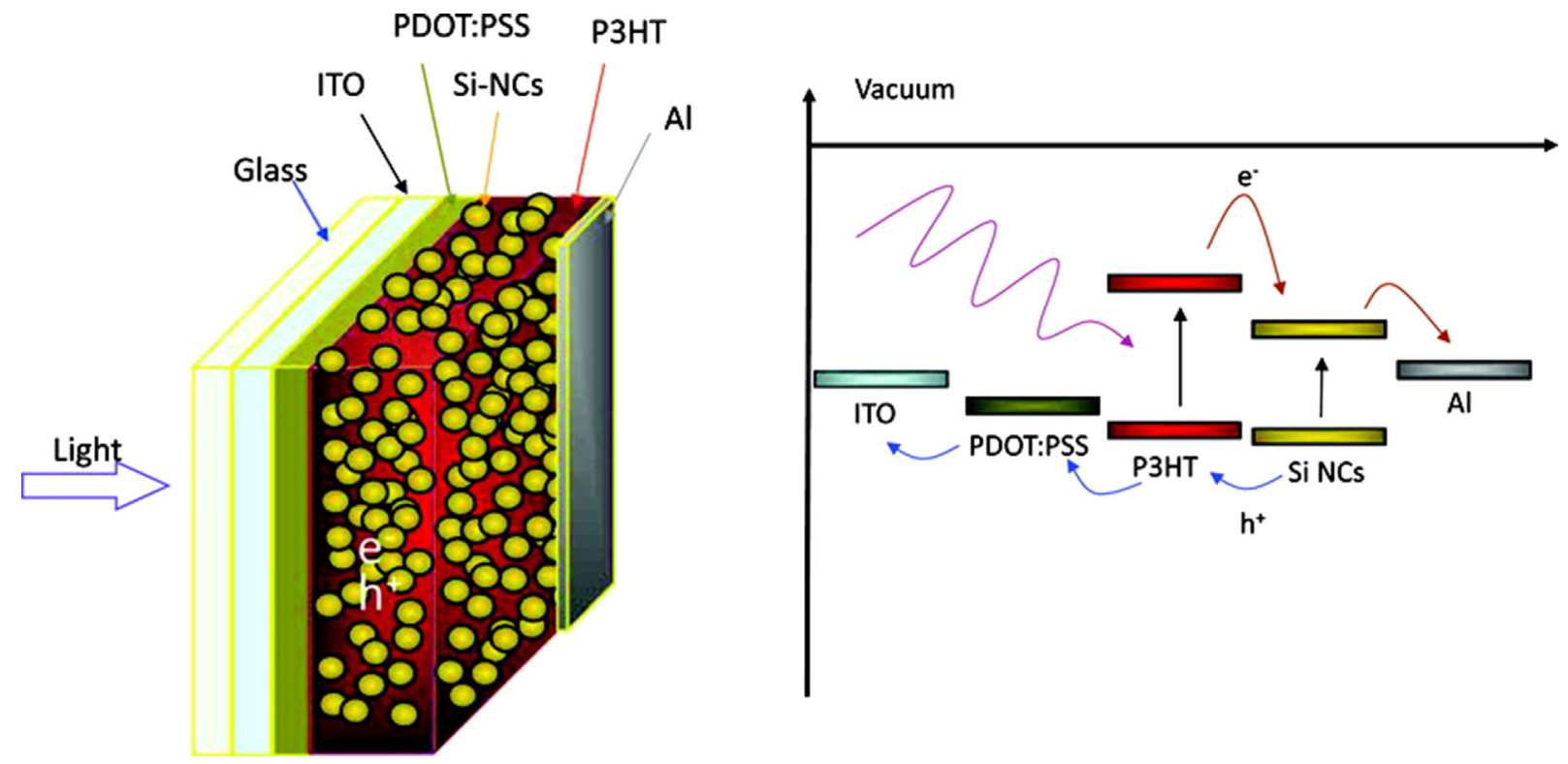

Figure 19. Illustration of a hybrid solar cell based on Si-NPs synthesized in a non-thermal plasma. In this concept, Si-NPs act as the electron transport medium while $\mathrm{P}_{3} \mathrm{HT}$ act as the hole transport medium as seen from the schematic diagram at the right. (Reprinted with permission from Liu et al. ${ }^{[220]}$ Copyright 2009, American Chemical Society.)

individual absorption by Si-NPs $(\sim 370 \mathrm{~nm}$ region, about one-third reduction in reflection), and absorption by the porous structure of Si-NP films (>500 nm region, film porosity in the range $60-90 \%)$. Authors also noted that, the down-shifting efficiency (DSE) should be at least $40 \%$ to contribute to the solar cell efficiency, otherwise, the downshifting becomes an efficiency-reducing process. Twenty percent DSE of Si-NPs used in this work ${ }^{[54,55]}$ therefore counters the porosity enhancement, and the increase of the external quantum efficiency is not proportional to the sunlight absorption at short wavelength region. They also reported that the degree of porosity and the film thickness are the determinant parameters on the efficiency increase of solar cells. The thicknesses below $60 \mathrm{~nm}$ and above $150 \mathrm{~nm}$ resulted in an increased reflectance of the solar cell. When Si-NP coated solar cells are left at room temperature in air for 11 days, the gained efficiency enhancement was observed to decrease from 3.7 to $1.4 \%$. Further in time, the enhancement was stabilized at $\sim 1.4 \%$. This is related with the oxidation of Si-NP surfaces, which decreases the refractive index of the material, and therefore, increasing the reflectance. This work concluded that the improvement in the solar cell performance was due to the improved light absorption of Si-NPs, rather than down-shifting.

In their second report, Pi et al. ${ }^{[196]}$ demonstrated that spin coating of SiNPs on solar cells improved the efficiency both due to increased absorption from porous Si-NP films and spectrum downshifting from individual Si-NPs. In this work, hydrosilylated Si-NPs, originally synthesized in a non-thermal plasma were inkjet-printed on a commercially finished multicrystalline Si solar cell. Authors used a multicrystalline Si solar cell instead of a monocrystalline Si solar cell because of the fact that multicrystalline Si solar cells have poorer response in short wavelength region $(<400 \mathrm{~nm})$, and the contribution of efficiency enhancement of Si-NPs can be more pronounced. PL measurements demonstrated that Si-NPs absorb photons at $370 \mathrm{~nm}$
Figure 2O. (a) $J-V$ characteristics of Si-NP/P3HT solar cells, and $\mathrm{P}_{3} \mathrm{HT}$ solar cells (inset). Solar cells that contain Si-NPs have a current density two times more than that of $\mathrm{P}_{3} \mathrm{HT}$ solar cells. (b) IPCE of Si-NP/P3HT solar cells in the range $400-700 \mathrm{~nm}$. (Reprinted with permission from Liu et al. ${ }^{[220]}$ Copyright 2009, American Chemical Society.) 
a)
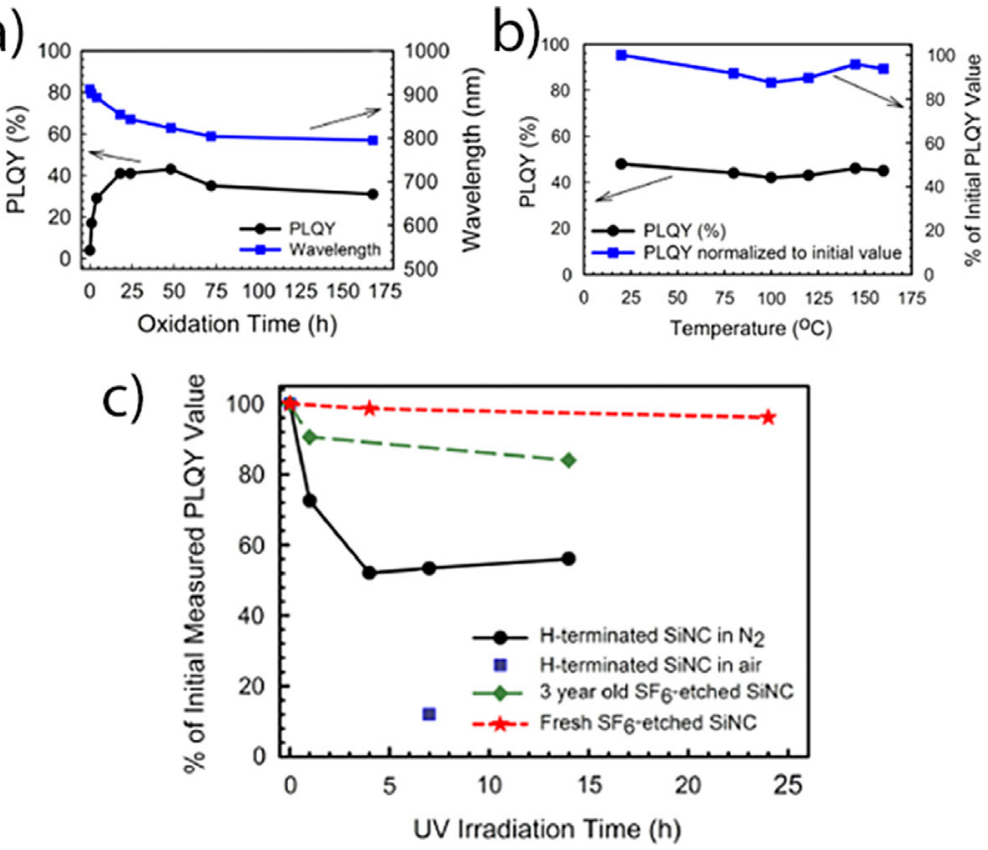

Figure 21. PL quantum yield and $\mathrm{PL}$ peak wavelength position of $\mathrm{Si}-\mathrm{NPs}$ of $\mathrm{SF}_{6}$-etched $\mathrm{Si}$ NPs (a) as a function of ambient oxidation time, and (b) as a function of temperature during thermal treatment. (c) Change of the PL quantum yield from its initial value as a function of UV irradiation time in air and in $\mathrm{N}_{2}$ ambient. (Reprinted with permission from Liptak et al. ${ }^{[221]}$ Copyright 2012, IOP Publishing. All rights reserved.)

wavelength and emit them at $773 \mathrm{~nm}$ with a PLOY of $53 \%$. This observation already indicates that Si-NPs behave as efficient down-shifters. It is reported that the efficiency enhancement can be maximized by adjusting the drop spacing and the printer head angle during inkjet printing of Si-NPs. The highest efficiency enhancement was found as $2 \%$, which increased the absolute external efficiency of the commercial multicrystalline solar cell from 17.2 to $17.5 \%$ (Figure 22). Reflectance of Si-NP-printed solar cells decreased in the range $300-400 \mathrm{~nm}$ and $640-1100 \mathrm{~nm}$, which is due to the improved absorption of porous Si-NP films and spectrum down-shifting of individual Si-NPs. In parallel with the decreased reflectance, EOE was observed to increase (Figure 23). An important remark here is that, the measured efficiency enhancement of multicrystalline $\mathrm{Si}$ solar cell was obtained without complicated work, thanks to the one-step synthesis of free-standing Si-NPs in the plasma. This is in contrast to other reports on using Si-NPs as down-shifters, where Si-NPs were embedded in matrices during synthesis, resulting in an unclarified efficiency enhancement mechanism or even a decrease in the efficiency. ${ }^{[206,223]}$

Hybrid solar cells have become popular in the past decade as they bring the advantages of organic and inorganic materials together. A remarkable observation of using Si-NPs in hybrid solar cells is that Si-NPs improve the current generation rate with respect to full-organic solar cells without Si-NPs. This was demonstrated by Herrmann et al. ${ }^{[224]}$ that using Si-NPs (they also used poly-Si thin films) as the electron acceptors in hybrid P3HT/Si-NP films in comparison to the full-organic P3HT/PCBM ([6,6]phenyl- $\mathrm{C}_{61}$-butyric acid methyl ester) films. Hybrid films improved the charge separation efficiency during photo-induced charge carrier generation about two times, due to the higher dielectric constant of Si with respect to P3HT. Rapid charge separation enabled faster transport of the generated electrons away from the interface, and hence, improved power conversion efficiency in solar cell operations. In addition, authors observed that mixing P3HT with Si-NPs improved the total absorption of the hybrid films about $20 \%$ with respect to P3HT in the region $300-1100 \mathrm{~nm}$-as a result of the improved absorption of SiNPs -without modifying the structure of P3HT.

Another example of hybrid solar cells with Si-NPs was demonstrated by the group of Nozaki. Ding et al. ${ }^{[225]}$ synthesized Si-NPs in a non-thermal plasma using $\mathrm{SiCl}_{4}$ as the precursor gas in the size range $2-10 \mathrm{~nm}$, which finally resulted in chlorinated surface of Si-NPs. They produced SiNP/P3HT solar cells, where Si-NPs were incorporated in the active layer. Similar to the other hybrid Si-NP/P3HT solar cells, Si-NPs acted as electron acceptor layer and P3HT acted as electron donor layer (Figure 24). The short circuit current density is observed to increase with incorporation of Si-NPs from $\sim 0.02 \mathrm{~mA} \mathrm{~cm}^{-2}$ (for the case of no Si-NPs) to $0.8 \mathrm{~mA}$ $\mathrm{cm}^{-2}$ (for the case of $50 \mathrm{wt} \% \mathrm{Si}-\mathrm{NPs}$ ), see Figure 25. The open circuit voltage and the fill factor were not affected, however, the power conversion efficiency was observed with the same increasing trend as the short circuit voltage with increased Si-NP weight ratios. At weight ratios higher than $50 \%$ of Si-NPs, the device performance deteriorated dramatically as a result of inhomogeneous dispersion and agglomeration of Si-NPs. The origin of the increased short circuit current up to $50 \mathrm{wt} \%$ arises from the absorption increase of the solar cell due to the presence of Si-NPs (below $350 \mathrm{~nm}$ ) and improved exciton dissociation efficiency at the Si-NP/P3HT interface, contributing to the generated current (in the range $350-600 \mathrm{~nm}$ ). Overall, the external quantum efficiency of the solar cells were observed to increase from $\sim 1.5$ to $\sim 13$ (arbitrary units) with increased amount of Si-NPs (maximum of 50\%) in the hybrid solar cells. The similarity of the results obtained from this study suggests 

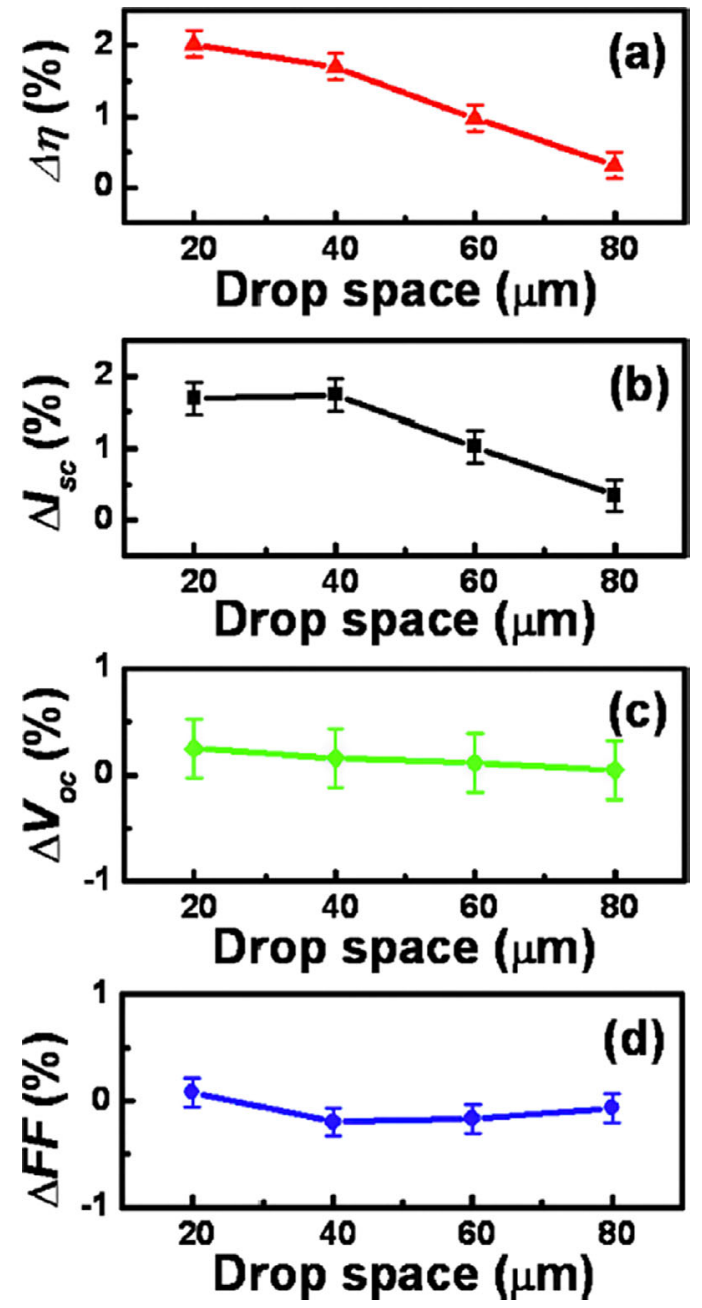

Figure 22. Characteristics of Si-NP inkjet-printed solar cells. (a) Efficiency, (b) short circuit current, (c) open circuit voltage, and (d) fill factor change as a function of drop space. (Reprinted with permission from Pi et al. ${ }^{[196]}$ Copyright 2012, American Chemical Society.)

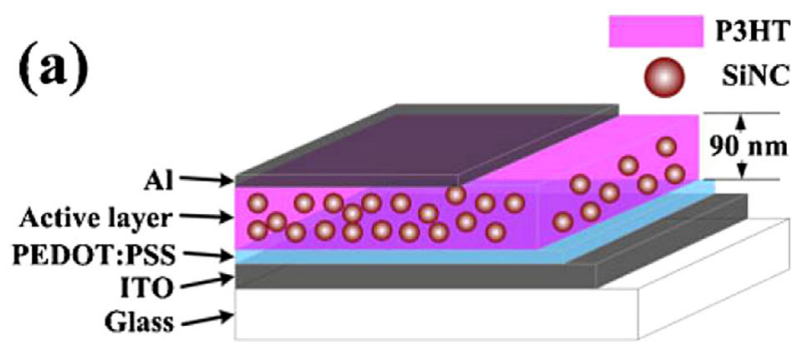

(b)

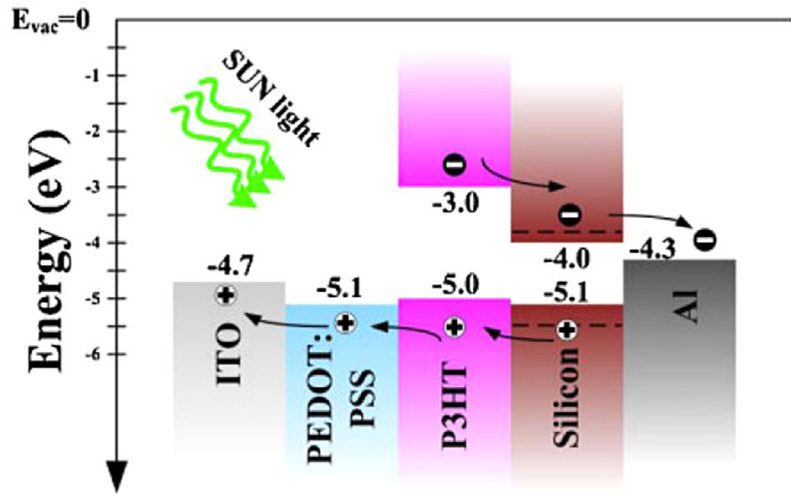

Figure 24. (a) Schematics and (b) the band alignment of bulkheterojunction $(\mathrm{BHJ})$ solar cells containing Si-NPs. (Reprinted with permission from Ding et al. ${ }^{[225]}$ Copyright 2013, The Japan Society of Applied Physics.)

that the valence band position of chlorine terminated Si-NPs (synthesized using $\mathrm{SiCl}_{4}$ precursor) are similar to that of hydrogen terminated Si-NPs (synthesized using $\mathrm{SiH}_{4}$ precursor). ${ }^{[220]}$

In this section, we presented the recent results on the solar cell application of plasma-synthesized Si-NPs. Although the current status of the research is in the lab-scale, some of the highlighted approaches are not far from being commercial. To our opinion, the concept of advanced photon management shows great potential to realize improved efficiency of solar cells. High PL quantum yield of Si-NPs and the possibility of integration of SiNPs on any type of solar cell without an electrical contact makes this technology highly feasible in the near future. The concept of hybrid solar cells is another promising route to proceed. Hybrid solar cells, in which Si-NPs act as electron acceptors, are shown to yield higher efficiencies with respect to their full-organic counterparts. Finally, embedding Si-NPs as active light absorbing material in single- and multi-junction tandem solar cells remains to be the greatest challenge. Although the theoretical
Figure 23. External quantum efficiency of solar cells with (red) and without (black) inkjet-printed Si-NPs for (a) $20 \mu \mathrm{m}$ and (b) $80 \mu \mathrm{m}$ drop space during inkjet printing. Blue lines are calculated EOE values obtained only by considering the antireflection of Si-NP films. (Reprinted with permission from $\mathrm{Pi}$ et al. ${ }^{[196]}$ Copyright 2012, American Chemical Society.) 


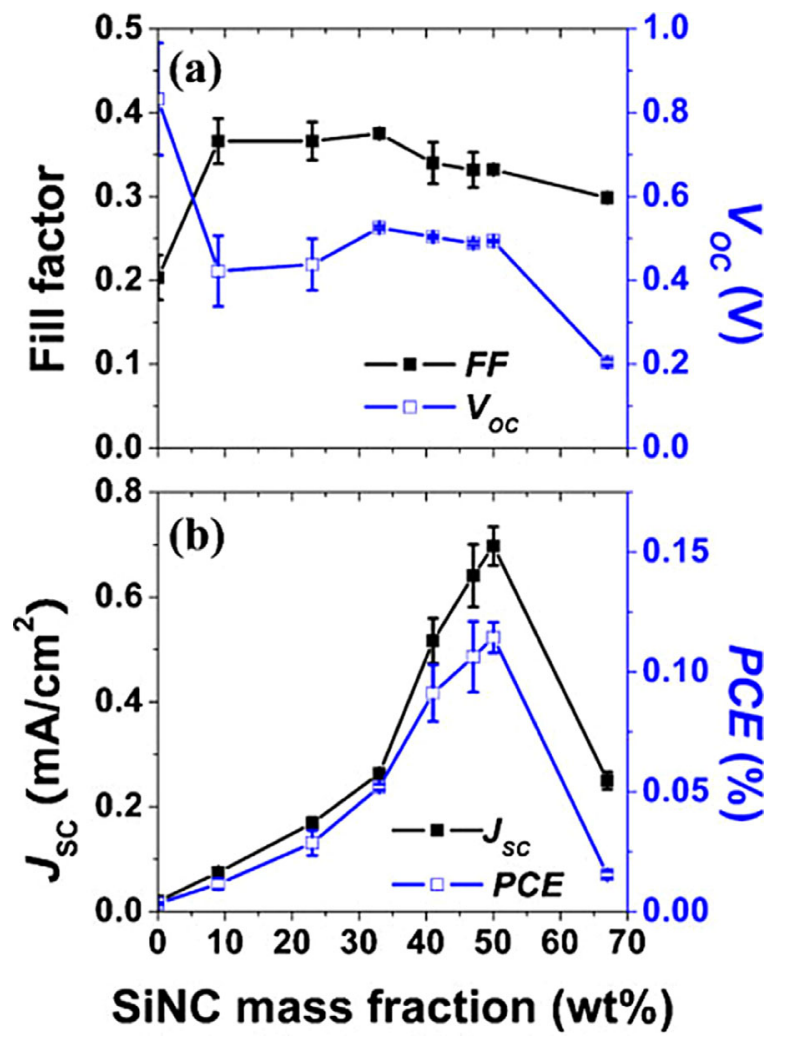

Figure 25. Ding et al. (a) Fill factor, open circuit voltage, (b) short circuit current, and PCE of BHJ solar cells as a function of Si-NP mass fraction. (Reprinted with permission from Ding et al. ${ }^{[225]}$ Copyright 2013, The Japan Society of Applied Physics.)

efficiencies exceed the Shockley-Queisser limit, these solar cells are far from reaching the efficiencies of $c$-Si solar cells.

\subsection{Batteries}

Ultra-high capacity lithium-ion batteries (LIBs) providing a high energy density over an extensive period of time is a key requirement to realize a wide range of future applications. ${ }^{[26]}$ Current LIB anode-electrodes are based on graphite with capacities of $375 \mathrm{mAhg}^{-1},{ }^{[227]}$ which are considered as insufficient to power the applications requiring high energy density with longevity. Research on ultra-high capacity lithium-ion batteries demonstrate that replacing standard carbon-based anodes with siliconbased materials enhances the anode capacity almost an order of magnitude, up to $\sim 4000 \mathrm{mAhg}^{-1}$. $^{[28,229]}$ On the other hand, experimental reports on bulk $c$-Si demonstrated that, the capacity of bulk c-Si is much lower than the theoretical estimation, in the range about two to threefolds of a commercial graphite electrode. ${ }^{[15]}$ This is due to the large volume expansion of almost $300 \%$ of silicon during lithiation, which introduces mechanical defects reducing the charge retention dramatically even before reaching a few tens of charge/discharge cycles. ${ }^{[14,230]}$

To reduce the effects of mechanical damage that results in the loss of charge retention and stability, the use of nanostructured silicon was proposed. Nano-sized silicon structures are shown to overcome the deteriorating effects of volumetric expansion as a result of their ability to relax the stress, ${ }^{[14]}$ thanks to their significantly increased surfaceto-volume ratio. Various silicon nanostructures with high cycle lifes have been demonstrated in the literature. Some of the examples are silicon nanotubes, ${ }^{[231]}$ nanowires, ${ }^{[232]}$ nanoparticles, ${ }^{[14]}$ porous structures, ${ }^{[233]}$ and silicon nanostructure based composite materials. ${ }^{[234-236]}$ In general, as the size of Si-NPs decreases, their charge storing capacity increases. ${ }^{[237]}$ In addition, the volume expansion is managed to a great extent when the average size of nanoparticles gets smaller ${ }^{[238]}$ due to the increased energy barrier for crack propagation at smaller sizes, a sizedependent feature known as Griffith's criterion. ${ }^{[89]}$ Still, Si-NPs with various sizes up to tens of nanometers can promote high charge storing capacity up to $2000 \mathrm{mAh} \mathrm{g}^{-1}$. 237,239$]$

Nevertheless, electrochemical reactions occurring at the Si-NP anode-electrolyte interface form an electrode/solidelectrolyte interface (SEI) layer via decomposition of the electrolyte salt on Si-NP surfaces. ${ }^{[100]} \mathrm{SEI}$ is considered as one of the major reasons for the capacity loss of Si-NPs: it hinders the lithium-ion transport, traps lithium irreversibly, and dramatically reduces the cycle life of Si-NPs. ${ }^{[100,240]}$ Moreover, SEI formed after initial lithiation is unstable, it cracks as a result of Si-NP swelling, and freshly-exposed regions of Si-NP surfaces to electrolyte provoke continual SEI growth ${ }^{\left[{ }^{19]}\right.}$ SEI is demonstrated to degrade the capacity of Si-NPs from 4000 to below $500 \mathrm{mAh} \mathrm{g}^{-1}$ within only 50 cycles $^{[241]}$ (while stable functionality of hundreds of cycles is expected from the commercial point of view). Therefore, realizing stable, and high energy density Si-NP anodes remains as one of the biggest challenges on realizing Si-NP based commercial LIBs.

Possibilities to realize nano silicon-based LIBs are then closely linked with a good understanding/control of the lithiation mechanism of silicon and formation of unstable SEI. Embedding Si-NPs in low volumetric expansion matrices, and realizing nano silicon-based composite materials to reduce the overall volumetric expansion of the anode by still achieving a reasonably high capacity are the hot topics of the research. In this context, gas-phase plasma synthesis routes take their advantage to their ability to synthesize compound nanomaterials, and feasibility to produce matrix embedded nanoparticles. Promising results have been observed especially using composite Si-NPs. In the following, we will highlight some of the key results reported within the last decade. At the end of this section, we summarize all the discussed results in Table 1 to 


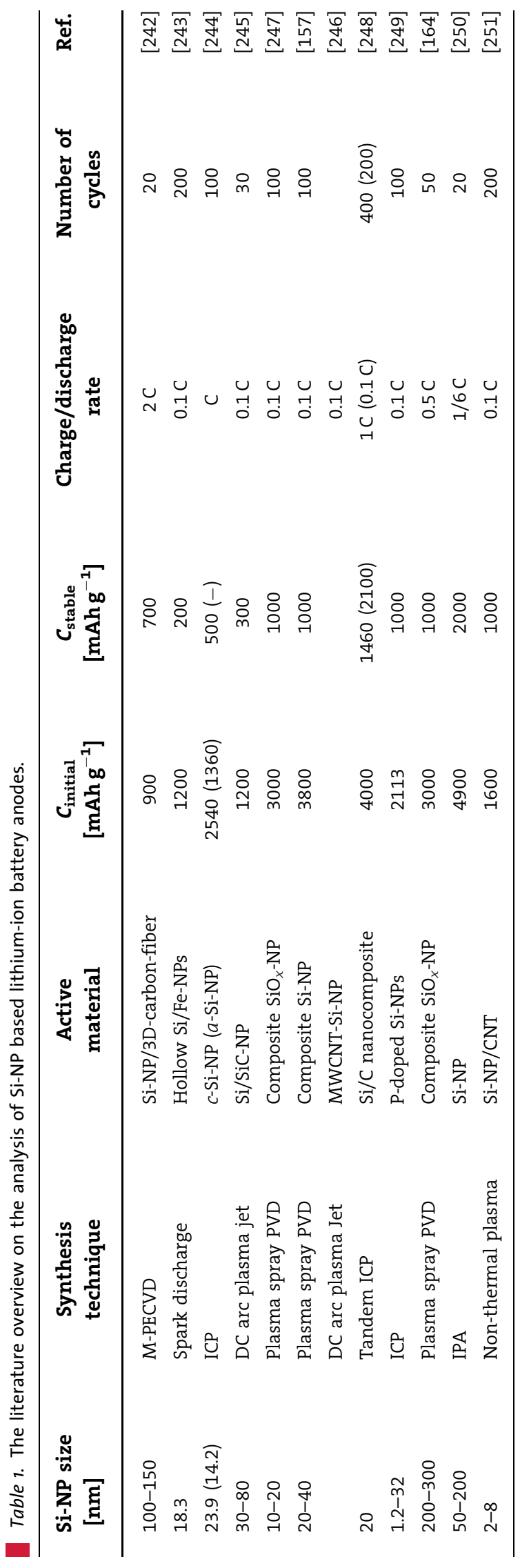

give an overview of the state-of-the-art results using gasphase plasma synthesized Si-NPs.

Wolf et al. ${ }^{[242]}$ took advantage of combining Si-NPs with 3D-carbon-fiber substrates in order to manage the volumetric expansion of Si-NPs better with lithium insertion. three-dimensional structure provided increased surface area for deposition of Si-NPs, together with mechanical stability and electrical conductivity improvement. Si-NPs with primary particle sizes of $27 \mathrm{~nm}$ were directly synthesized in a microwave plasma enhanced chemical vapor deposition tool using $\mathrm{Ar} / \mathrm{H}_{2} / \mathrm{TCS}$ (trichlorosilane) mixture. For the electrochemical measurements, various Si-NP/3D-carbon-fiber weight ratios were prepared by controlling the deposition time of Si-NPs. Both 3Dcarbon-fiber substrate and Si-NPs were used as the active material. During the synthesis, thermal treatment of 900 on the substrate level induced sintering of Si-NPs and they were able to grow up to micrometer-size range. Authors reported that with increased weight ratio of Si-NPs, first cycle reversible capacity improved up to $\sim 1400 \mathrm{mAh}^{-1}$ (for 34 wt\% Si-NPs at charge/discharge rate C/20). Further increase in the weight ratio dramatically reduced the reversible capacity (for $54 \mathrm{wt} \%$ Si-NPs, reversible capacity was below $10 \%$ ) as a result of disintegration of micrometersized agglomerates after excessive volumetric expansion. For a Si-NP content less than $20 \mathrm{wt} \%$, stable capacity was $700 \mathrm{mAh}^{-1}$ for tens of cycles at higher charge/discharge rates up to $2 \mathrm{C}$.

Hollow nanoparticles of Si caught particular interest for the case of LIB anodes because of their low density and increased porosity, which facilitates the volumetric changes more efficiently. Byeon et al. ${ }^{[243]}$ synthesized hollow Si/Fe alloy nanoparticles in an ambient heterogeneous spark discharge in a $\mathrm{N}_{2}$ atmosphere. Synthesized particles were in the mean size of $18.3 \mathrm{~nm}$ with a geometric standard deviation of $1.24 \mathrm{~nm}$ (Figure 26). The capacity of $\mathrm{Si} / \mathrm{Fe}-\mathrm{NPs}$ were observed as $1200 \mathrm{mAh}^{-1}$ initially, and then it dropped to $900 \mathrm{mAh} \mathrm{g}^{-1}$, staying stable in this value for 200 cycles. Authors concluded that the hollow structure improved the lifetime of the electrode, and the porosity improved the ion transport, resulting in enhanced cycling performances.

Jang et al. ${ }^{[244]}$ studied the cycling performances of amorphous and crystalline Si-NPs synthesized in a double tube inductively coupled plasma discharge reactor at low $(100 \mathrm{~W})$ and high $(500 \mathrm{~W})$ plasma powers. The average size of the nanoparticles were 14.2 and $23.9 \mathrm{~nm}$ for the amorphous and crystalline Si-NPs, respectively. The as-synthesized amorphous and crystalline Si-NPs were analyzed in terms of their cycling performances in coin cells. In order to demonstrate the improved cycling performance of Si-NPs based on their nano-size, they also demonstrated the cycling performance of Si microparticles. The initial capacity of microparticles was $\sim 2750 \mathrm{mAh} \mathrm{g}^{-1}$ 

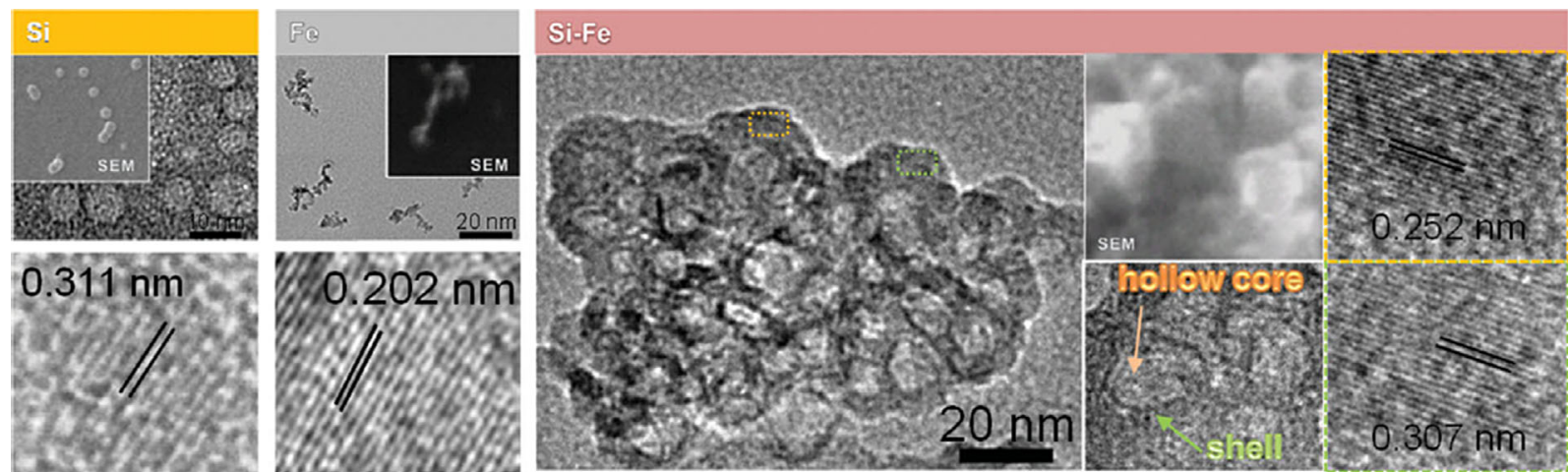

Figure 26. TEM and SEM images of Si-NPs, Fe-NPs, and Si/Fe-NPs. (Reprinted with permission from Byeon et al. ${ }^{[243]}$ Copyright 2014 , Wiley-VCH Verlag.)

and rapidly decreased to $115 \mathrm{mAh}^{-1}$ at the end of 100 cycles. In the case of crystalline Si-NPs, the initial capacity was observed as $2540 \mathrm{mAh} \mathrm{g}^{-1}$. After a rapid decrease of the capacity, a stable capacity of $\sim 500 \mathrm{mAh}^{-1}$ was achieved from 30th cycle till the end of 100th cycle. On the other hand, amorphous Si-NPs started at a rather lower initial capacity of $1360 \mathrm{mAh} \mathrm{g}^{-1}$ and without exhibiting a rapid initial decrease, the capacity is decreased down to $500 \mathrm{mAh} \mathrm{g}^{-1}$ after 100 cycle (Figure 27). The authors related the lower initial capacity of amorphous Si-NPs to larger amount of oxide phase in them. On the other hand, they also noted that the oxide phase ( $\mathrm{Li}-\mathrm{Si}-\mathrm{O}$ ) prevented an initial rapid decrease of the capacity, enabling higher Coulombic efficiency with respect to crystalline Si-NPs.

Another example of the cycling performance of Si- and C-based composite nanoparticles was reported by Jeon et al. ${ }^{[25]}$ They synthesized Si/SiC-NP composites in a DC arc discharge using $\mathrm{CH}_{4} / \mathrm{SiH}_{4}$ gas mixture. For different ratios of
$\mathrm{CH}_{4} / \mathrm{SiH}_{4}$, the initial and further capacities were investigated. It was shown that (Figure 28), although the highest first cycle capacity is observed from pure Si-NPs $\left(2400 \mathrm{mAh} \mathrm{g}^{-1}\right)$, in the long term (20 cycles), nanoparticles exhibited improved Coulombic efficiency in parallel with the increase of the carbon content in SiC-NPs (380 $\mathrm{mAh} \mathrm{g}^{-1}$ for the highest carbon content). The reason of this improved Coulombic efficiency from SiC-NPs is due to the presence of the buffer carbon layer, which ensures stable solid-electrolyte interface (SEI) formation. In addition, presence of carbon in the composite nanoparticles, reduced the volumetric expansion with respect to the case of pure Si-NPs during lithiation.

Si-NPs were also combined with other types of carbonbased nanomaterials, such as carbon nanotubes (CNTs). CNTs are particularly interesting composite materials for Si-NP based anodes because of their relatively improved specific capacity of $600 \mathrm{mAh}^{-1}$ with respect to graphite,

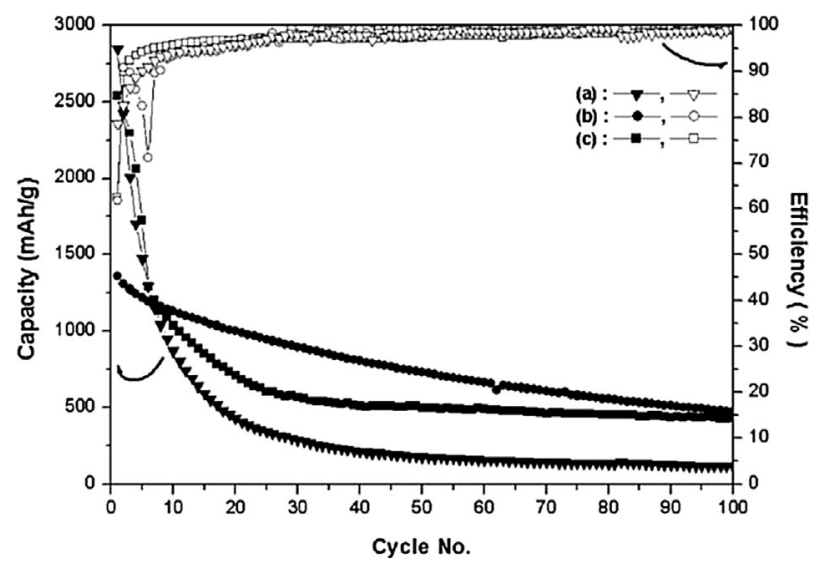

Figure 27. Cycling performance and Coulombic efficiency of anodes prepared using (a) crystalline microparticles of $\mathrm{Si}$, (b) amorphous Si-NPs, and (c) crystalline Si-NPs. (Reprinted with permission from Jang et al. ${ }^{[24]}$ Copyright 2014, Wiley-VCH Verlag.)

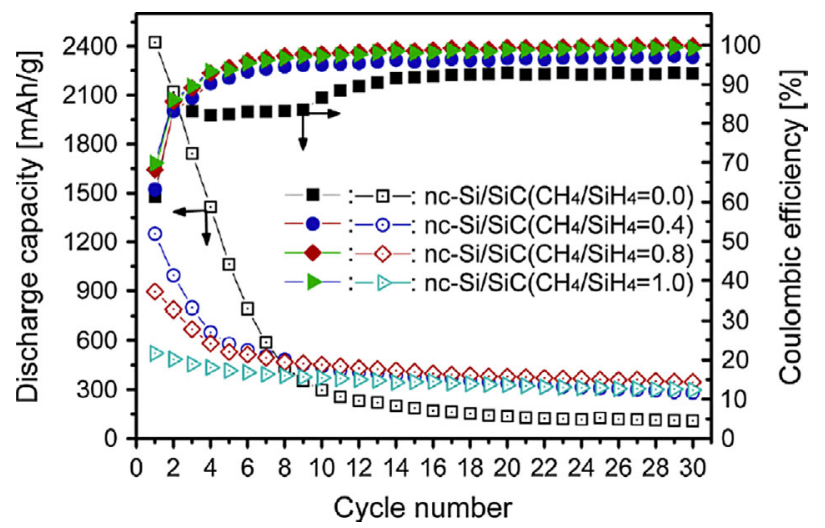

Figure 28. Cycling performance and Coulombic efficiency of anodes prepared using Si/SiC-NP composites for different $\mathrm{CH}_{4} /$ $\mathrm{SiH}_{4}$ molar ratios. (Reprinted with permission from Jeon et al. ${ }^{[245]}$ Copyright 2014, Elsevier.) 

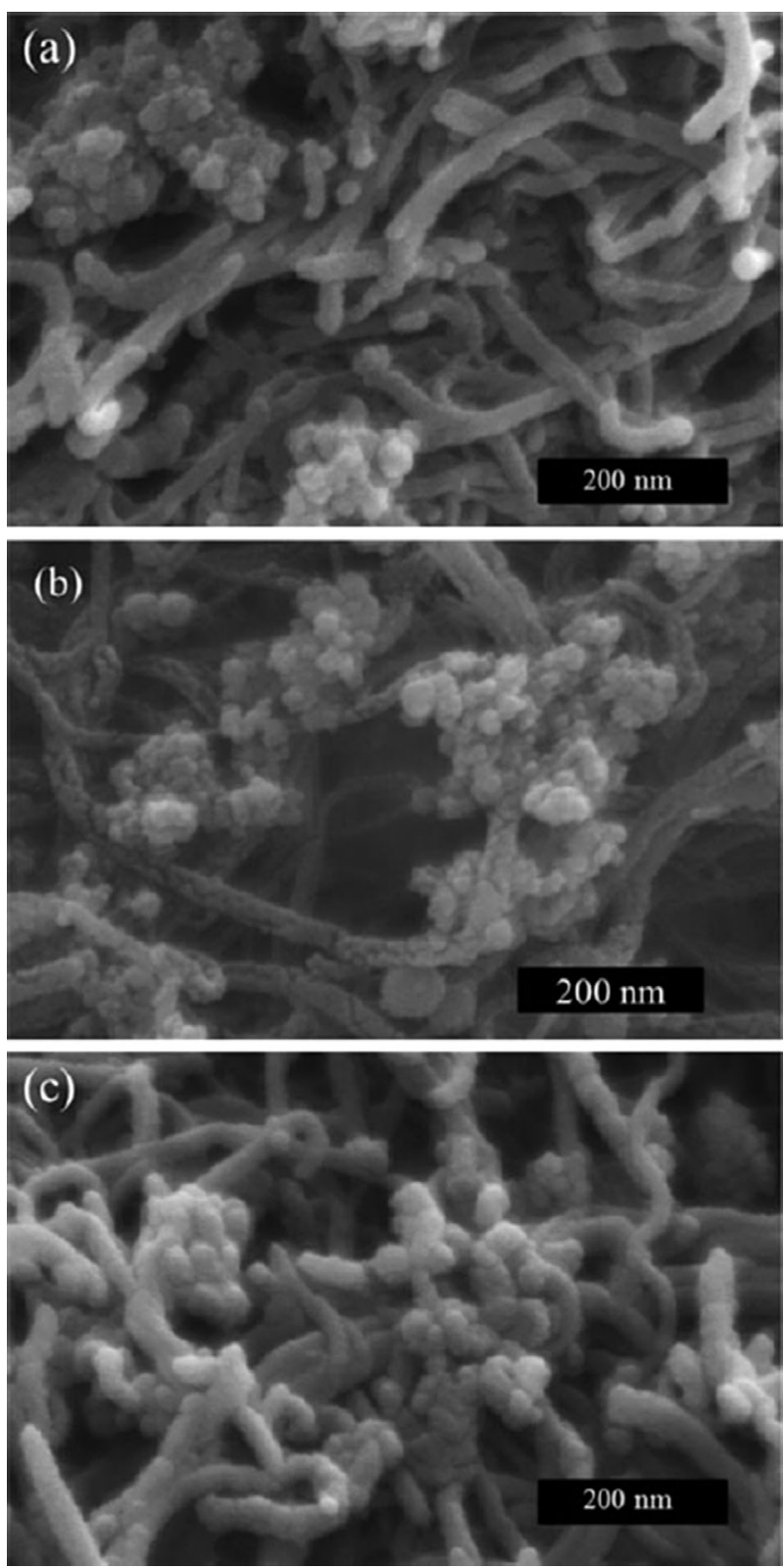

Figure 29. Field-emission SEM (FE-SEM) images of MWCNT/Si-NP composites synthesized at different input powers of (a) 10.2 (b) 11.3 and (c) $12.6 \mathrm{~kW}$. (Reprinted with permission from Na et al. ${ }^{[246]}$ Copyright 2014, Wiley-VCH Verlag.)

and because of providing vast amount of volume to buffer the expansion of Si-NPs during lithiation without pulverizing the electrode. $\mathrm{Na}$ et al. ${ }^{[246]}$ synthesized multi-walled carbon nanotubes (MWCNTs) and Si-NPs as composite materials in a thermal DC arc plasma jet from the raw materials of commercial MWCNTs and micrometer-sized Si power. They controlled the density ratio of MWCNTs and Si-NPs by tuning the plasma power and varying the injection location of Si particles (Figure 29). Although they did not demonstrate the cycling performances of synthesized materials in the cells, they suggested that the material could be readily used in the cycling experiments.

Thermal plasma tools are shown to be suitable for synthesizing Si-NPs and composite Si-NPs with high throughput and with compatibility for battery applications. The group of Kambara and Yoshida performed a series of studies on nanocomposite Si-NPs synthesized in thermal plasmas. In one study Kambara et al. ${ }^{[157]}$ synthesized composite Si-NPs from raw silicon powders with starting averages sizes of $20 \mu \mathrm{m}$ in an $\mathrm{Ar} / \mathrm{H}_{2}$ plasma. In addition, $\mathrm{CH}_{4}$ was also injected to synthesize composite Si-NPs for achieving various $\mathrm{C} / \mathrm{Si}$ molar ratios, i.e., $0,1.0,1.5,3.0$. Energy dispersive spectroscopy (EDX) measurements demonstrated a core-shell structure of the synthesized material: Si-NPs were in the full crystalline form and locate at the core of the nanoparticles, while they were surrounded by an amorphous C shell. The initial capacity of Si-NPs were observed as $3800 \mathrm{mAh} \mathrm{g}^{-1}$ and this capacity was observed to decrease down to $1140 \mathrm{mAh}^{-1}$ with increasing $\mathrm{C} / \mathrm{Si}$ molar ratio from 1.0 to 3.0 (Figure 30). The overall reduction of the initial capacity is due to the presence of SiC-NPs in the synthesized powder, lowering the overall capacity and also reducing the electric conduction. Normalized specific capacity per effective Si weight demonstrated that C/Si ratios of 1.0 and 1.5 actually had higher capacity than pure Si-NPs $(\mathrm{C} / \mathrm{Si}=0)$, however the lower overall capacity is due to the presence of SiC-NPs as discussed above. Increased capacity of core/shell Si-NPs is a result of reduced irreversible capacity loss because of the presence of amorphous C layer on Si-NPs, forming a stable SEI. For $\mathrm{C} / \mathrm{Si}=3.0$, the excess amount of SiC-NPs deteriorate the

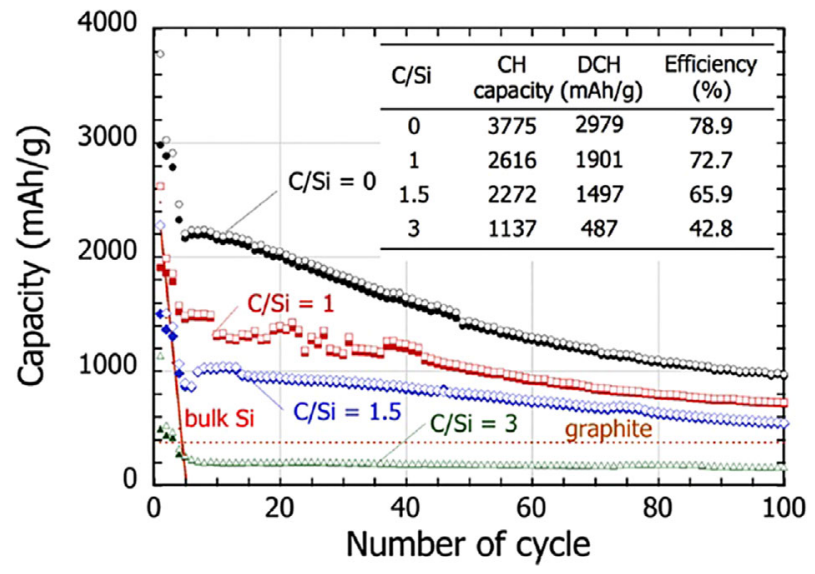

Figure 30. Cycling performance of nanocomposite Si-NPs with different $\mathrm{C} / \mathrm{Si}$ molar ratios. Solid and open marks are for the capacities after lithiation delithiation, respectively. (Reprinted with permission from Kambara et al. ${ }^{[157]}$ Copyright 2014, AIP Publishing LLC.) 
electrical conduction paths despite the electrochemically favorable surface of these core/shell Si-NPs.

In another study, Homma et al. ${ }^{[247]}$ synthesized composite $\mathrm{SiO}_{\chi}$ nanostructures in a DC-RF hybrid ICP system. Several micrometer-sized SiO powders were fed through a plasma source where $\mathrm{Ar} / \mathrm{H}_{2}$ gas mixture is used to generate a plasma. Completely evaporated SiO powders recondensate in the expanding and rapidly cooling zone to form agglomerated nanoparticles with primary sizes of 10-20 nm. Similar to the previous study, $\mathrm{CH}_{4}$ was also added to achieve different $\mathrm{C} / \mathrm{Si}$ molar ratios. Electron energy loss spectroscopy (EELS) revealed that for pure $\mathrm{SiO}_{x}$ nanoparticles, the core is composed of crystalline Si-NPs while the outer region is occupied by amorphous $\mathrm{SiO}_{\chi}$ shell as a result of disproportionation reactions. Furthermore, it was observed from EELS and XRD that increasing the molar fraction of $\mathrm{C} / \mathrm{Si}$ improves the crystal fraction and leads to formation of highly crystalline $\mathrm{SiO}_{x} / \mathrm{Si}-\mathrm{NPs}$ (with increased core size and reduced shell size), together with individual Si-NPs and SiC-NPs. The initial capacities of composite nanoparticles with $\mathrm{C} / \mathrm{Si}=0.25$ and 1.0 were observed as $3000 \mathrm{mAh} \mathrm{g}^{-1}$, decaying down to $1000 \mathrm{mAh} \mathrm{g}^{-1}$ after 100 cycles, and for $\mathrm{C} / \mathrm{Si}=0$ and 1.5 were observed as $2000 \mathrm{mAh} \mathrm{g}^{-1}$ (lower than the initial capacity of raw SiO, which is about $2500 \mathrm{mAhg}^{-1}$ ), decaying down to $600 \mathrm{mAh}^{-1}$. The authors discussed that increasing the size of the crystalline Si-NP core with C/Si molar ratio reduces the thickness of amorphous $\mathrm{SiO}_{x}$ shell. For $\mathrm{C} / \mathrm{Si}-=0.25$ and 1.0 the capacities were found to increase with respect to $\mathrm{C} / \mathrm{Si}=0$ case as a result of reduced amorphous shell. However, the amorphous $\mathrm{SiO}_{x}$ shell is necessary to obtain a stable form of SEI layer $\left(\mathrm{Li}_{4} \mathrm{SiO}_{4}\right)$, which also prevents excessive expansion of the Si-NP core. For the case of the thinnest $\mathrm{SiO}_{x}$ layer $(\mathrm{C} / \mathrm{Si}=1.5)$, the SEI layer was below the optimum thickness, and the capacity was degraded similar to the case of $\mathrm{C} / \mathrm{Si}=0$, where the amorphous layer was more than the optimum thickness, which formed excessive SEI, trapping a significant amount of lithium ions in the form of $\mathrm{Li}_{4} \mathrm{SiO}_{4}$. Differential capacity analyses of all types of composite nanoparticles demonstrated that, although the pristine samples were electrochemically behaving different, they exhibit very similar electrochemical behavior after 100 cycles.

In an electrochemical analysis study, Kambara et al. ${ }^{[164]}$ investigated the electrochemical impedance characteristics of $\mathrm{Si} / \mathrm{SiO}_{x}$ core/shell composite nanoparticles with an addition of $\mathrm{CH}_{4}$ gas (C/Si molar ratio of 0.25 ) during synthesis. The cells with stable capacities of $1000 \mathrm{mAh} \mathrm{g}^{-1}$ (for 50 cycles) were analyzed by means of electrochemical impedance spectroscopy (EIS). They concluded that addition of $\mathrm{CH}_{4}$ reduced the surface resistance of $\mathrm{Si} / \mathrm{SiO}_{x}$ core/ shell composite nanoparticles because of the formation of $\mathrm{Li}_{2} \mathrm{O}$ on the $\mathrm{SiO}_{x}$ shell surface. Since $\mathrm{CH}_{4}$ has a role on the formation of $\mathrm{Si} / \mathrm{SiO}_{x}$ core/shell phase from $\mathrm{SiO}$ via disproportionation reactions, the reduced surface resistance is linked with the use of $\mathrm{CH}_{4}$ during synthesis.

An important requirement of realizing efficient Si/C nanocomposites is to control their mixture in order to have homogeneous buffering against volumetric expansion and equally improved conductivity throughout the nanocomposite anode. Chang at.al. ${ }^{[28]}$ used a tandem plasma reaction (TPR) method to synthesize $\mathrm{Si} / \mathrm{C}$ nanocomposites by sputtering $\mathrm{Si}$ targets and either decomposing $\mathrm{CH}_{4}(\mathrm{Si} /$ $\mathrm{C}-\mathrm{CH}_{4}$ ) or sputtering graphite (Si/C-graphite) in ICP downstream plasma zone. Nanocomposites were observed to have a homogeneous size distribution of $20 \mathrm{~nm}$ with a carbon fraction of $10 \mathrm{wt} \%$ for both $\mathrm{Si} / \mathrm{C}-\mathrm{CH}_{4}$ and $\mathrm{Si} /$ C-graphite. XPS analyses demonstrated that $\mathrm{Si} / \mathrm{C}$-graphite samples were composed of elemental silicon and carbon. On the other hand, $\mathrm{Si} / \mathrm{C}-\mathrm{CH}_{4}$ samples were observed as $\mathrm{Si}$ NPs with fully SiC-covered surfaces. CV analyses demonstrated the lithiation and delithiation of $\mathrm{Si} / \mathrm{C}$ (Si/C-graphite)
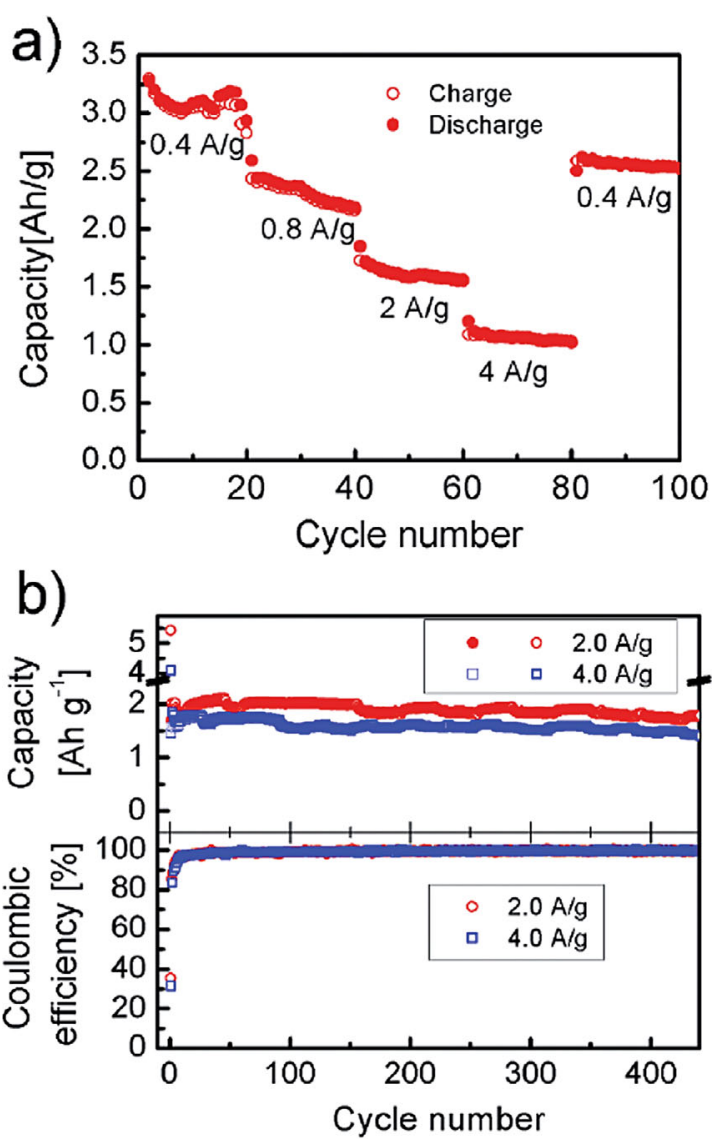

Figure 31. (a) Cycling performance of Si-NP/C-graphite nanocomposites (with 10 wt\% carbon in a half-cell) at various charge/discharge current densities. (b) The capacity (upper panel) and the Coulombic efficiency (lower panel) of the nanocomposites shown in (a) at a current density of 2.0 and 4. $\mathrm{OAg}^{-1}$. (Reprinted with permission from Chang et al. ${ }^{[248]}$ Copyright 2014, The Royal Society of Chemistry.) 
nanocomposites in a clear way. However, CV results of $\mathrm{SiC}$ $\left(\mathrm{Si} / \mathrm{C}-\mathrm{CH}_{4}\right)$ nanocomposites suggest that these nanocomposites were inactive to lithiation. Cyclic voltammetry analyses also demonstrated the distinct cycling behavior of $\mathrm{Si} / \mathrm{C}$ and $\mathrm{SiC}$ nanocomposites. While $\mathrm{Si} / \mathrm{C}$ exhibit capacities greater than $2000 \mathrm{mAh} \mathrm{g}^{-1}$ at a charge/discharge rate of $0.1 \mathrm{C}$ even after 200 cycles (Figure 31), SiC nanocomposites exhibit dramatically lower capacities of about $200 \mathrm{mAh} \mathrm{g}^{-1}$ right after the first few cycles. When the charge/discharge rate is $1 \mathrm{C}$, a capacity of $1500 \mathrm{mAh} \mathrm{g}^{-1}$ was observed after 400 cycles from $\mathrm{Si} / \mathrm{C}$ nanocomposites. It is notable that these cells were directly prepared from the synthesized material, without any binder. This work demonstrated that one of the key challenges on synthesizing high performance $\mathrm{Si} / \mathrm{C}$ nanocomposites is to inhibit the formation of SiC, which dramatically reduces the performance of the Li-ion battery.

Doping nanostructures of $\mathrm{Si}$ is another approach for improving the capacity of Si-nanostructure based anodes. Jung et al..$^{[249]}$ synthesized P-doped Si-NPs using an ICP with double tube reactor in a $\mathrm{PH}_{3} / \mathrm{Ar}$ and $\mathrm{SiH}_{4} / \mathrm{Ar}$ gas mixture. They found that doping Si-NPs with P within the solubility limit of $10^{21}$ atoms $\mathrm{cm}^{-1}$ did not bring an improvement to the cycling performance. On the other hand, overdoping Si-NPs with P resulted in formation of an additional phase in the active material, and $\mathrm{P}$ atoms separately undergo lithiation reactions forming $\mathrm{Li}_{3} \mathrm{P}$, a reversible form of lithiation. This resulted in improved initial capacity of $2113 \mathrm{mAhg}^{-1}$, and also improved the overall capacity during 100 cycles of lithiation/delithiation from $\sim 750$ to $\sim 1000 \mathrm{mAh}^{-1}$. They concluded that, overdoping
Si-NPs with P increased the capacity of the anodes, due to the formation of the reversible $\mathrm{Li}_{3} \mathrm{P}$ phase, rather than improving the electrical conductivity.

Another study on thermal plasma synthesized Si-NPs from raw Si powders was performed by Leblanc et al. ${ }^{[250]}$ Si-NPs were synthesized using induced plasma atomization (IPA) technique from raw Si particles. Synthesized Si-NP powder had a size distribution of $50-200 \mathrm{~nm}$ with an average size of $85 \mathrm{~nm}$. Assembled coin cells exhibited an exceptionally high capacity of $4900-\mathrm{mAhg}^{-1}$ initially under a charge/discharge rate of $\mathrm{C} / 24$. After the initial discharge, $12 \%$ of lithium is irreversibly lost, due to the formation of the SEI. Later on, at a charge/discharge rate of C/6, Si-NP coin cells exhibited a capacity of $\sim 2000 \mathrm{mAh} \mathrm{g}^{-1}$ after 20 cycles. However, the observed profile did not reach a stable Coulombic efficiency, and resembled a decrease further with additional charging, which indicated a continual SEI growth as a result of volumetric expansion during cycling of Si-NPs.

Zhong et al. ${ }^{[251]}$ reported that homogeneous dispersion of Si-NPs, less than $10 \mathrm{~nm}$ in size, in CNTs and a polymer binder (polyvinylpirrolidone, PVP) enable long term electrochemical stability. In their work, non-thermal plasma synthesized and functionalized Si-NPs were mixed in liquid-phase with CNTs and PVP, and then annealed at $500^{\circ} \mathrm{C}$ to form an electrochemically stable film. It was observed from SEM and TEM images that individual Si-NPs form agglomerates of tens of nanometers in an amorphous carbon matrix (Figure 32). The initial capacity of cells was observed around $1600 \mathrm{mAh}^{-1}$, decaying quickly to $1000 \mathrm{mAh} \mathrm{g}^{-1}$ and staying at similar values for 200 cycles. Coulombic
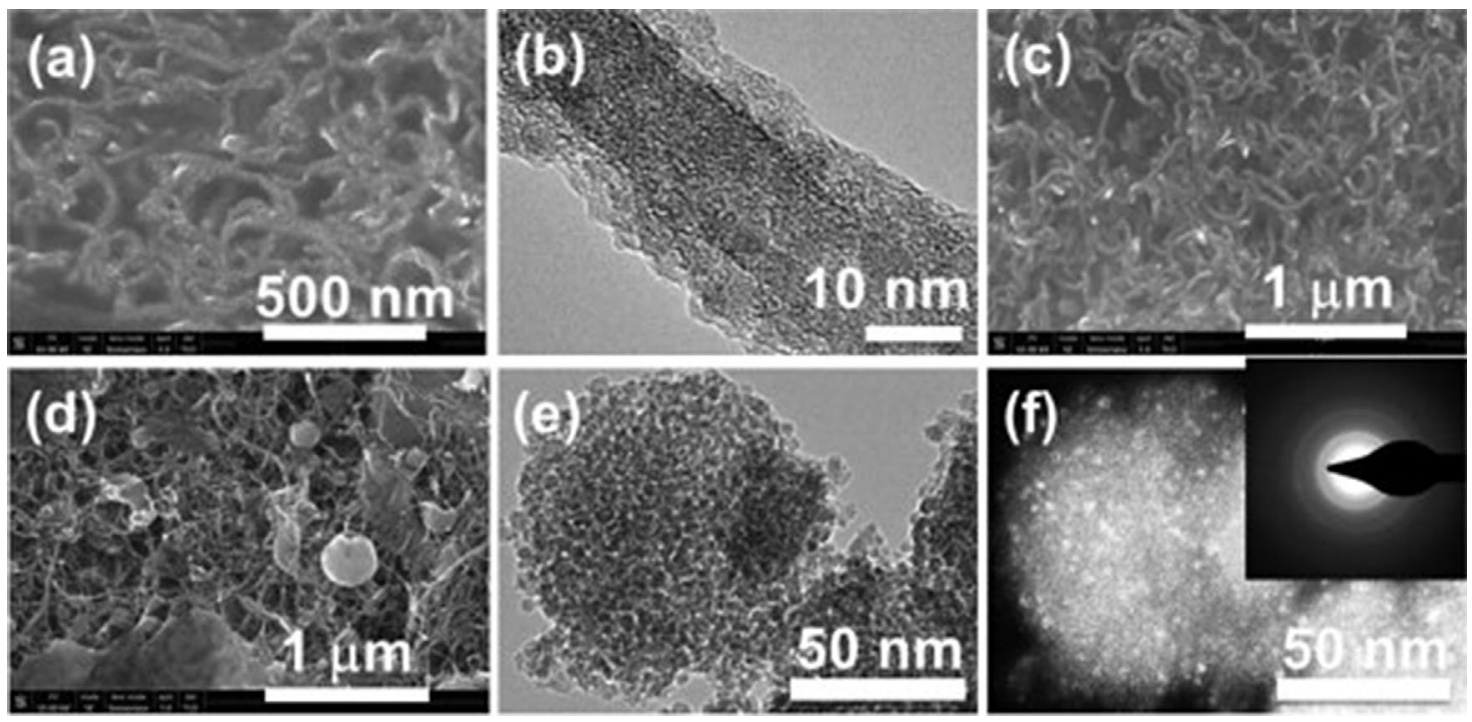

Figure 32. (a) SEM image of a mixture of Si-NPs, CNTs, and PVP. (b) TEM image of Si-NPs and PVP on a MWCNT. (c) SEM image of CNTs and PVP, without Si-NPs. (d) Annealing of the sample shown in (a) at $550^{\circ} \mathrm{C}$ in argon atmosphere. (e) TEM and (f) dark field images of Si-NPs after annealing them at $550^{\circ} \mathrm{C}$. Inset of $(f)$ shows the selected area electron diffraction (SAED) pattern of Si-NPs. (Reprinted with permission from Zhong et al. ${ }^{[251]}$ Copyright 2015, Elsevier.) 
efficiencies were observed as $99.8 \%$, demonstrating the long term stability of these anodes. In a series of controlled experiments, the authors have concluded that functionalizing the surface of Si-NPs does not essentially improve the electrochemical performance, but it helps preventing sedimentation of nanoparticles during solution processing. They have also concluded that PVP is critical for achieving a long term stability in battery performance. Furthermore, annealing PVP-containing coatings leads to the formation of amorphous carbon -and amorphous silicon carbide -around Si-NPs. The reason of the initial low capacity was then linked with the presence of silicon carbide phase.

In summary, LIB applications of Si-NPs appear to show great promise due to their very high charge storing capacity. Some of the state-of-the-art Si-NP anodes mentioned here already outperform the carbon-based anodes in commercial LIBs up to hundreds of charge/discharge cycles. The main challenge here is to further improve the cycling lifetime of Si-NP anodes. This can be achieved by gaining a deeper understanding on the processes that take place during the lithiation of Si-NPs, namely, the volumetric expansion, and excessive SEI formation. Engineering solutions like embedding Si-NPs in low expansion coefficient anode materials are shown to improve the cycling lifetime significantly.

\section{What is Next? Further Applications, Opportunities, and Challenges}

In this review, we summarized the recent advancements on gas-phase plasma synthesis of Si-NPs and their use in solar cell and lithium-ion battery applications. Gas-phase plasma synthesis routes enable size- and surface-controlled Si-NP production together with high throughputs, which are critical requirements for industrial scale applications. These Si-NPs offer great potential to promote improved efficiency solar cells and high capacity LIBs. However, to our opinion, two important energy technologies await a breakthrough by the involvement of gas-phase plasma synthesized Si-NPs. The energy technologies we mention here are the (photo) electrochemical water splitting, and thermoelectrics.

\subsection{Water Splitting}

(Photo) electrochemical water splitting for hydrogen production attracts a great deal of attention from the perspective of catalysis, energy storage, and fuel cell applications. A number of studies demonstrated that $\mathrm{Si}$ nanostructures can be efficiently used to produce hydrogen via (photo) catalytic reactions. ${ }^{[101-104,252-254]}$ In addition, water can be split chemically using Si-NPs, without a need of light, heat, electricity, or external agents. ${ }^{[18]}$ Recent research on the catalytic properties of Si-NPs have revealed that Si-NPs react with water and produce hydrogen up to 1000 times faster than bulk $c-\mathrm{Si}^{[18]}$ and up to ten times faster than competing metal composites like $\mathrm{Al}^{[255]}$ and $\mathrm{Zn} .{ }^{[256]} \mathrm{A}$ broad size range of Si-NPs can react with water to produce hydrogen. ${ }^{[18,103,252,257]}$ However, the etch rate and hydrogen production rate of Si-NPs dramatically increase at smaller sizes as a result of increased surface area and more isotropic nature of the etching at smaller scales. ${ }^{[18]}$ With these reported high hydrogen generation rates, even higher than metal nanoparticles, Si-NPs can potentially be of practical use for "on-demand" hydrogen generation, which can lead to realization of liquid hydrogen fuel cells for portable power applications.

In this aspect, plasmas can play a critical role on realizing higher production efficiencies and more controlled material properties. One of the benefits that plasmas can bring on the water splitting technology is the vast amount of possibilities on surface functionalization of Si-NPs. For instance, a density functional theory study by Zhang et al. ${ }^{[19]}$ indicated that hydrogen and chlorine terminated Si-NWs are promising photocatalysts as such surface functionalization separates HOMO and LUMO of Si-NWs and prevents electron-hole recombination during photocatalytic reaction. This type and other types of surface functionalization routes -we have already highlighted some of the key examples -can feasibly be done in plasmas during Si-NP synthesis. Another benefit of using plasmas for Si-NP synthesis is the ability to make films with controlled porosity and primary particle size together with high throughput. This feature can potentially realize one-step production of photocathodes -with an option to have functionalized surfaces -for hydrogen production cells compatible to industrial scale production.

\subsection{Thermoelectric Applications}

One of the first demonstrations of Si-based thermoelectrics was reported by Dismukes et al. ${ }^{[258]}$ in the form of a $\mathrm{Si}_{0.85} \mathrm{Ge}_{0.15}$ alloy. They reported a dimensionless figure of merit $Z T$ of 0.8 and 1.0 for heavily doped p-type and n-type alloys, respectively. Since then, there is an increased amount of research on Si-based alloys -especially in the nanostructured form. ${ }^{[259,260]}$ Already at the beginning of the research, it was understood that having a reduced thermal conductivity is not only enough, but improving the electrical conductivity of $\mathrm{Si}$ is a requirement to achieve high $Z T$ values, at least around 1. For achieving higher electronic conductivity, doping ${ }^{[112,261-268]}$ and surface functionalization $^{[269,270]}$ became important steps to reach higher $Z T$ values using Si-based nanostructures. One of the most studied system is SiGe alloy with improved $Z T$ values of up to 1.3 (at $1775 \mathrm{~K}$ ) for $\mathrm{n}$-type $\mathrm{Si}_{0.8} \mathrm{Ge}_{0.2 \cdot} \cdot{ }^{[262]}$ Apart from that, 
$\mathrm{Mg}_{2} \mathrm{Si}$ and $\mathrm{Mn}_{15} \mathrm{Si}_{26}$ thermoelectric materials were reported with $Z T$ values of 0.6 and 0.7 (at $775 \mathrm{~K}$ ), respectively. Although the observed $Z T$ values are lower than the stateof-the-art achievements using $\mathrm{Bi}_{2} \mathrm{Te} / \mathrm{Sb}_{2} \mathrm{Te}_{3}$ thermoelectric materials $(Z T \sim 2.4),{ }^{[113]}$ affordability, availability, and nontoxicity of Si made this material an important candidate for future thermoelectric applications.

Plasmas bring a number of advantages on the Si-based thermoelectrics. First of all, the possibility to functionalize and dope Si-NPs during the synthesis can reduce the processing time, widen the available options in terms of functionalizing agents, and result improved thermoelectric properties. It was already shown that Si-NPs can be doped in plasmas using phosphine $\left(\mathrm{PH}_{3}\right)^{[109,271,272]}$ and trimethylphospite $\left(\mathrm{P}\left(\mathrm{OCH}_{3}\right)_{3}\right)^{[273]}$ for $\mathrm{n}$-type doping, and using diborane $\left(\mathrm{B}_{2} \mathrm{H}_{6}\right)^{[109]}$ for p-type doping. These Si-NPs and their alloys exhibit $Z T$ values up to 0.85 (at $1273 \mathrm{~K}$ ), ${ }^{\left[{ }^{[27}\right]}$ which are comparable to the nanomaterials synthesized in environments other than plasmas. Another advantage of using plasmas is the possibility to synthesize a wide variety of alloys during the process. Such kind of doped-alloy Si-based nanoparticles include $\mathrm{Si} / \mathrm{Ge}$ and $\mathrm{Si} / \mathrm{WSi}_{2}{ }^{[272]}$ Together with high throughput, doping, and alloying in one-step process brings a major time- and cost-efficiency over other techniques, which are using multiple steps to dope and alloy nanostructures. To our opinion, thus, plasmas might play a significant role for thermoelectric applications of Si-NPs considering the critical advantages they bring.

\subsection{Plasma Modeling: Improved Understanding on Formation and Transport of Si-NPs in Plasmas}

One of the important challenges to establish better control on the morphology and the size distribution of Si-NPs is to establish an in-depth understanding of the formation and transport of Si-NPs in plasmas, by taking the specific type of plasma and reactor geometry into account. In this sense, although it is beyond the scope of this review, the importance of theoretical guidance to the experimental investigations should be addressed. We should note that, there are only a few groups devoting serous effort on plasma modeling of nanoparticles. As a result, comparing the state-of-the-art results in experimental observations of nanoparticle formation in plasmas, the modeling work is lagging behind. Modeling plays an important role on tackling a number of challenges, which mostly remain unresolved from the perspective of experimental work. These challenges are initial phase of nanoparticle formation, further growth of nanoparticles via nucleation and/or coagulation, transport of nanoparticles in plasmas under the effect of flow dynamics.

The work of modeling of the formation of nanoparticles from the first monomers, their homogeneous nucleation, and coagulation was pioneered by the group of Girshick and by the group of Kushner. Girshick's group have studied the nanoparticle formation process in thermal plasmas using discrete-sectional models and moment-type models. They investigated the time-dependent aerosol models for the homogeneous nucleation of nanoparticles from a supersaturated vapor including the dependence of the final size and number of nanoparticles on the cooling rate and the initial precursor concentration. ${ }^{[158,159,274]}$ They also investigated the ion-induced nucleation of nanoparticles from dissociated molecules in thermal plasmas. ${ }^{[159]}$ The group of Kushner have focused on the role of negative ions on the formation of ionic clusters, which are the seeds of nanoparticles, in electropositive RF discharges. Using Monte Carlo hybrid models and particle-in-cell (PIC) simulations, they revealed the role of negative ions on clustering in plasmas, where the negative ions increase the residence time of ionic clusters by trapping them, leading to the formation of nanoand micro-sized particles. ${ }^{[275-278]}$ They have also investigated the electron transport coefficients in dusty plasmas. ${ }^{[279]}$

Important efforts on understanding the effect of flow dynamics on the size distribution and morphology of nanoparticles have been put by the group of Colombo. They investigated the effect of reactor geometry, and the effect of flow dynamics at different parts of an inductively coupled thermal plasma nanoparticle synthesis tool using fluid dynamics models in order to achieve control on nanoparticle size distribution and improve the throughput of the process. ${ }^{[156,160]}$ They have also studied on idealized reactor geometries in order to eliminate the background recirculation patterns, where poor quality of nanoparticles were synthesized, and diffusion of nanoparticles to the wall was observed. ${ }^{[161]}$ For improving the size distribution control of Si-NPs synthesized in ETP, we have also modeled the flow dynamics and mass transport at the downstream of the reactor. By using continuum fluid dynamics model, we have observed a significant amount of mass transport to the recirculation cells, which is the origin of the formation of large Si-NPs with sizes in the range $50-120 \mathrm{~nm}$. By also modeling the spatial confinement of the central plasma beam, we demonstrated the mitigation of the mass flow to the recirculation cells. In this case, formation of large Si-NPs were prevented to a great extent and mostly small Si-NPs (with sizes in the range 2-10 $\mathrm{nm}$ ) synthesized in the central plasma beam were experimentally observed. ${ }^{[48]}$ This work demonstrated how to design an idealized reactor geometry in order to produce size controlled Si-NPs with size distributions in the range less than $10 \mathrm{~nm}$.

\section{Conclusion}

In this paper we reviewed the gas-phase plasma synthesis of free-standing Si-NPs, their formation mechanisms, and their 
use in future technological applications. In addition, we reviewed the plasma sources and environments, which are used for Si-NP synthesis, and their characteristic features.

About the plasma environments used to synthesize Si-NPs we can draw four main conclusions:

1) Plasmas meet a number of critical demands on effective synthesis of Si-NPs. Plasmas enable the control and modification of the morphology, size, size distribution, and functionalization of Si-NPs at the same time, a major advantage over other synthesis methods. In addition, the free-standing morphology for easy manipulation, synthesis of high-melting-temperature materials and their alloys, and doping them simultaneously are other benefits that plasmas bring. The possibility of upscaling and high throughput processing is an opportunity from a commercial point of view. The challenge here is to keep the lab-scale synthesis parameters optimized for the fab-scale plasmas after upscaling -possibly with novel reactor designs.

2) Si-NP growth mechanism is not unique, and cannot be generalized to all plasmas. We showed that the way of dissociation of the precursor, the lifetime of ions and radicals, and the residence time distributions in a plasma critically affects the Si-NP growth mechanisms. In plasmas with "hot" electrons, electron-induced dissociation of the precursor enables anion-induced growth pathway, while in the case of low energy electrons, $\mathrm{Ar}^{+}$ion-induced dissociation of the precursor enables cation-induced growth pathway. In addition, radical-induced growth can become a contributing growth pathway if radical residence times are similar to the nanoparticle growth times in the plasma.

3) It is hard to name a particular plasma technique as "the ultimate" Si-NP synthesis tool for every application. Low volume plasmas (like atmospheric pressure microplasmas and non-thermal plasmas) are able to produce Si-NPs with well-controlled size distributions, however with a lower throughput. On the other hand, high volume, high power plasmas (like microwave plasmas, remote plasmas and thermal plasmas) are able to reach dramatically higher throughputs but have less control over the distribution of sizes. Depending on the application, a particular plasma synthesis tool could become more advantageous with respect to other plasma synthesis tools. For solar cell and photoelectrochemical applications, direct size distribution control is a critical requirement, where high throughput synthesis using high volume, high power plasmas will possibly be followed by a size-separation step. On the other hand, for LIB and thermoelectric applications, size distribution could be sacrificed to some extent (by staying below the maximum critical size) to take the full advantage of high throughput low cost processing.
4) Developing a reliable material diagnostics technique is necessary in order to optimize the plasma parameters during Si-NP synthesis. Although the topic of characterization techniques of plasma synthesized Si-NPs is beyond the scope of this review, its importance cannot be underrated, and should be mentioned here. Diagnostic techniques are not only critical for characterizing the synthesized Si-NPs, but also directly necessary to optimize and control the plasma parameters in order to obtain a specific Si-NP size and/or surface chemistry. In this aspect, size and surface chemistry analysis techniques are critically important. The most common methods for the size analysis of Si-NPs are transmission electron microscopy (TEM), x-ray diffraction (XRD), and photoluminescence spectroscopy (PL). For the surface chemistry analysis, Fourier transform infrared spectroscopy (FTIR) is commonly used. In a series of recent publications, we have demonstrated that Raman spectroscopy can be used as a standard diagnostic tool for the analysis of the size distribution ${ }^{[187]}$ and surface chemistry ${ }^{[193]}$ of Si-NPs. Especially on size distribution analysis, we have shown that, Raman spectroscopy is non-destructive and time-efficient, and gives reliable results as the analyses agree well with other size analysis techniques like TEM and PL. We indeed have shown that Raman spectroscopy helps us understand the growth mechanism and the formation region of Si-NPs in plasmas by analyzing the size distribution of synthesized Si-NPs with varying the plasma parameters. ${ }^{[47,48]}$

Apart from the plasma environments, we also discussed on the applications of plasma synthesized Si-NPs. Efficiency improvement of solar cells were clearly demonstrated using Si-NPs. However, the efficiency of solar cells using Si-NPs in the active layer is somewhat lower when compared to thin film and crystalline silicon solar cell technologies. We argue that a promising route to explore in the short term is to use Si-NPs in advanced photon management concepts. The studies already demonstrated that inclusion of a Si-NP down-sifter and/or a downconverter layer is a relatively simple process, and it clearly contributes to the already-achieved-efficiency of finished solar cells. An important advantage that plasmas bring is the in situ functionalization, which can enable long term stability of optical and electronic properties of Si-NPs.

For the case of LIB application of plasma synthesized Si-NPs, encouraging results have already been achieved. For further improvements on the battery performance, two challenges need to be tackled: facilitating the volumetric expansion, and achieving a reduced, stable SEI during lithiation of Si-NPs. For facilitating the volumetric expansion, various methods have been tried such as increasing the porosity of Si-NP network, embedding Si-NPs in low- 
expansion-coefficient soft amorphous matrices, and realizing composite Si-NPs to reduce the overall volumetric expansion. We argue that, although the formation of the SEI on Si-NP surfaces is one of the biggest challenges for stable operations, it is possibly the least explored and understood part of a battery. Encouraging efforts have been put into engineering solutions to prevent direct interaction of the electrolyte material with Si-NP surface by encapsulating Si-NPs in barrier layers. However, promoting a fundamental understanding of SEI formation on Si-NP surfaces is lacking. Limited number of studies in the literature are based on ex situ analyses, revealing the irreversible components of SEI at various steps of lithiation. In our opinion, real-time surface-sensitive in situ analysis techniques are necessary in order to fully understand the SEI formation on Si-NPs surfaces during lithiation. This would then reveal possible routes to limit SEI formation on Si-NPs.

We finally discussed the concepts of water splitting and thermoelectrics as the other possible future technological applications of Si-NPs. The recent research have demonstrated that Si-NPs have a great potential to dominate the future of these technologies, and plasmas can offer critical advantages to these applications such as surface functionalization to improve the efficiency of the water splitting rate, doping to improve the figure of merit value in thermoelectric applications, and high throughput processing for realizing industrial scale processing.

Acknowledgments: This work is part of the research programme of the Foundation for Fundamental Research on Matter (FOM), which is part of The Netherlands Organization for Scientific Research (NWO). Authors thank Katherina Dohnalova and Tom Gregorkiewicz from the University of Amsterdam for the optical analysis of carrier multiplication properties of Si-NPs, shown in Figure 1, synthesized in the remote expanding thermal plasma.

Received: October 30, 2015; Revised: November 25, 2015; Accepted: November 26, 2015; DOI: 10.1002/ppap.201500197

Keywords: downstream plasma; nanofabrication; nucleation; plasma polymerization; silicon nanoparticle

[1] L. T. Canham, K. G. Barraclough, D. J. Robbins, Appl. Phys. Lett. 1987, 51, 1509.

[2] O. King, D. Hall, Phys. Rev. B. Condens. Matter 1994, 50, 10661.

[3] K. Bothe, R. J. Falster, J. D. Murphy, Appl. Phys. Lett. 2012, 101, 032107.

[4] Y. Koshka, S. Ostapenko, I. Tarasov, S. McHugo, J. P. Kalejs, Appl. Phys. Lett. 1999, 74, 1555.

[5] S. K. Ray, S. Maikap, W. Banerjee, S. Das, J. Phys. D. Appl. Phys. 2013, 46, 153001.

[6] F. Maier-Flaig, J. Rinck, M. Stephan, T. Bocksrocker, M. Bruns, C. Kübel, A. K. Powell, G. A. Ozin, U. Lemmer, Nano Lett. 2013, 13, 475 .
[7] K.-Y. Cheng, R. Anthony, U. R. Kortshagen, R. J. Holmes, Nano Lett. 2011, 11, 1952.

[8] A. Marconi, A. Anopchenko, G. Pucker, L. Pavesi, Appl. Phys. Lett. 2011, 98, 201103.

[9] D. P. Puzzo, E. J. Henderson, M. G. Helander, Z. Wang, G. A. Ozin, Z. Lu, Nano Lett. 2011, 11, 1585.

[10] B.-H. Kim, C.-H. Cho, J.-S. Mun, M.-K. Kwon, T.-Y. Park, J. S. Kim, C. C. Byeon, J. Lee, S.-J. Park, Adv. Mater. 2008, 20, 3100 .

[11] S. Tiwari, F. Rana, H. Hanafi, A. Hartstein, E. F. Crabbé, K. Chan, Appl. Phys. Lett. 1996, 68, 1377.

[12] R. Rao, R. Steimle, M. Sadd, C. Swift, B. Hradsky, S. Straub, T. Merchant, M. Stoker, S. Anderson, M. Rossow, J. Yater, B. Acred, K. Harber, E. Prinz, B. White, R. Muralidhar, Solid. State. Electron. 2004, 48, 1463

[13] M. L. Ostraat, J. W. De Blauwe, M. L. Green, L. D. Bell, M. L. Brongersma, J. Casperson, R. C. Flagan, H. A. Atwater, Appl. Phys. Lett. 2001, 79, 433.

[14] J. Graetz, C. C. Ahn, R. Yazami, B. Fultz, Electrochem. SolidState Lett. 2003, 6, A194.

[15] W.-R. Liu, Z.-Z. Guo, W.-S. Young, D.-T. Shieh, H.-C. Wu, M.-H. Yang, N.-L. Wu, J. Power Sources 2005, 140, 139.

[16] Y. -M. Lin, K. C. Klavetter, P. R. Abel, N. C. Davy, J. L. Snider, A. Heller, C. B. Mullins, Chem. Commun. (Camb). 2012, 48, 7268.

[17] Y.-S. Hu, R. Demir-Cakan, M.-M. Titirici, J.-O. Müller, R. Schlögl, M. Antonietti, J. Maier, Angew. Chem. Int. Ed. Engl. 2008, 47, 1645.

[18] F. Erogbogbo, T. Lin, P. M. Tucciarone, K. M. Lajoie, L. Lai, G. D. Patki, P. N. Prasad, M. T. Swihart, Nano Lett. 2013, 12, 451.

[19] R. Zhang, X. Liu, Z. Wen, O. Jiang, J. Phys. Chem. C 2011, 115, 3425.

[20] F. Erogbogbo, K.-T. Yong, I. Roy, G. Xu, P. N. Prasad, M. T. Swihart, ACS Nano 2008, 2, 873.

[21] G. -H. G. Pan, A. Barras, L. Boussekey, X. Ou, A. Addad, R. Boukherroub, Langmuir 2013, 29, 12688.

[22] C.-Y. Liu, U. R. Kortshagen, Nanoscale Res. Lett. 2010, 5, 1253.

[23] X. Pi, Q. Li, D. Li, D. Yang, Sol. Energ. Mater. Sol. Cells 2011, 95, 2941.

[24] J. -W. Luo, P. Stradins, A. Zunger, Energ. Environ. Sci. 2011, 4, 2546.

[25] G. Conibeer, M. Green, R. Corkish, Y. Cho, E.-C. Cho, C.-W. Jiang, T. Fangsuwannarak, E. Pink, Y. Huang, T. Puzzer, T. Trupke, B. Richards, A. Shalav, K. Lin, Thin Solid Films 2006, 511-512, 654.

[26] I. Dogan, I. Yildiz, R. Turan, Phys. E Low-Dimens. Syst. Nanostructures 2009, 41, 976.

[27] M. Zacharias, J. Heitmann, R. Scholz, U. Kahler, M. Schmidt, J. Bläsing, Appl. Phys. Lett. 2002, 80, 661.

[28] K. Ma, J. Y. Feng, Z. J. Zhang, Nanotechnology 2006, 17, 4650.

[29] R. Gago, L. Vázquez, R. Cuerno, M. Varela, C. Ballesteros, J. M. Albella, Appl. Phys. Lett. 2001, 78, 3316.

[30] P. Mutti, G. Ghislotti, S. Bertoni, L. Bonoldi, G. F. Cerofolini, L. Meda, E. Grilli, M. Guzzi, Appl. Phys. Lett. 1995, 66, 851.

[31] U. Serincan, G. Aygun, R. Turan, J. Lumin. 2005, 113, 229.

[32] T. Shimizu-Iwayama, N. Kurumado, D. E. Hole, P. D. Townsend, J. Appl. Phys. 1998, 83, 6018.

[33] R. K. Baldwin, K. A. Pettigrew, J. C. Garno, P. P. Power, G. Liu, S. M. Kauzlarich, J. Am. Chem. Soc. 2002, 124, 1150.

[34] J. D. Holmes, K. J. Ziegler, R. C. Doty, L. E. Pell, K. P. Johnston, B. A. Korgel, J. Am. Chem. Soc. 2001, 123, 3743.

[35] J. Wilcoxon, G. Samara, P. Provencio, Phys. Rev. B 1999, 60, 2704.

[36] L. Mangolini, U. Kortshagen, Adv. Mater. 2007, 19, 2513. 
[37] U. Kortshagen, R. Anthony, R. Gresback, Z. Holman, R. Ligman, C.-Y. Liu, L. Mangolini, S. A. Campbell, Pure Appl. Chem. 2008, 80, 1901.

[38] Z. C. Holman, U. R. Kortshagen, J. Phys. D. Appl. Phys. 2011, 44, 174009.

[39] R. Gresback, Z. Holman, U. Kortshagen, Appl. Phys. Lett. 2007, 91, 093119.

[40] R. Gresback, T. Nozaki, K. Okazaki, Nanotechnology 2011, 22, 305605.

[41] Y. Ding, R. Yamada, R. Gresback, S. Zhou, X. Pi, T. Nozaki, J. Phys. D. Appl. Phys. 2014, 47, 485202.

[42] R. P. Chaukulkar, K. De Peuter, P. Stradins, S. Pylypenko, J. P. Bell, Y. Yang, S. Agarwal, ACS Appl. Mater. Interface. 2014, 6, 19026.

[43] B. N. Jariwala, N. J. Kramer, M. C. Petcu, D. C. Bobela, M. C. M. van de Sanden, P. Stradins, C. V. Ciobanu, S. Agarwal, J. Phys. Chem. C 2011, 115, 20375.

[44] M. Shiratani, K. Koga, S. Ando, T. Inoue, Y. Watanabe, S. Nunomura, M. Kondo, Surf. Coat. Technol. 2007, 201, 5468.

[45] K. Koga, T. Matsunaga, Y. Kim, K. Nakahara, D. Yamashita, H. Matsuzaki, K. Kamataki, G. Uchida, N. Itagaki, M. Shiratani, Jpn. J. Appl. Phys. 2012, 51, 01AD02.

[46] İ. Doğan, N. J. Kramer, R. H. J. Westermann, K. Dohnalova, A. H. M. Smets, M. A. Verheijen, T. Gregorkiewicz, M. C. M. van de Sanden, J. Appl. Phys. 2013, 113, 134306.

[47] İ. Doğan, S. L. Weeks, S. Agarwal, M. C. M. van de Sanden, J. Appl. Phys. 2014, 115, 244301.

[48] İ. Doğan, R. H. J. Westerman, M. C. M. van de Sanden, Plasma Sources Sci. Technol. 2015, 24, 015030.

[49] B. N. Jariwala, O. S. Dewey, P. Stradins, C. V. Ciobanu, S. Agarwal, ACS Appl. Mater. Interface. 2011, 3, 3033.

[50] S. L. Weeks, B. Macco, M. C. M. van de Sanden, S. Agarwal, Langmuir 2012, 28, 17295.

[51] G. Uchida, K. Yamamoto, Y. Kawashima, M. Sato, K. Nakahara, K. Kamataki, N. Itagaki, K. Koga, M. Kondo, M. Shiratani, Phys. Status Solidi 2011, 8, 3017.

[52] G. Uchida, K. Yamamoto, M. Sato, Y. Kawashima, K Nakahara, K. Kamataki, N. Itagaki, K. Koga, M. Shiratani, Jpn. J. Appl. Phys. 2012, 51, 01AD01.

[53] X. D. Pi, R. W. Liptak, S. A. Campbell, U. Kortshagen, Appl. Phys. Lett. 2007, 91, 083112.

[54] X. D. Pi, R. W. Liptak, J. Deneen Nowak, N. P. Wells, C. B. Carter, S. A. Campbell, U. Kortshagen, Nanotechnology 2008, 19, 245603.

[55] R. W. Liptak, B. Devetter, J. H. Thomas, U. Kortshagen, S. A. Campbell, Nanotechnology 2009, 20, 035603.

[56] P. F. Trwoga, A. J. Kenyon, C. W. Pitt, J. Appl. Phys. 1998, 83, 3789.

[57] W. D. A. M. de Boer, D. Timmerman, K. Dohnalová, I. N Yassievich, H. Zhang, W. J. Buma, T. Gregorkiewicz, Nat. Nanotechnol. 2010, 5, 878.

[58] M. Hybertsen, Phys. Rev. Lett. 1994, 72, 1514.

[59] C. Delerue, G. Allan, M. Lannoo, Phys. Rev. B 1993, 48, 11024.

[60] A. M. P. Botas, R. A. S. Ferreira, R. N. Pereira, R. J. Anthony, T. Moura, D. J. Rowe, U. Kortshagen, J. Phys. Chem. C 2014, 118, 10375.

[61] B. Goller, S. Polisski, H. Wiggers, D. Kovalev, Appl. Phys. Lett. 2010, 97, 041110.

[62] P. Shen, N. Uesawa, S. Inasawa, Y. Yamaguchi, J. Mater. Chem. 2010, 20, 1669

[63] A. Gupta, H. Wiggers, Nanotechnology 2011, 22, 055707.

[64] J. B. Miller, A. R. Van Sickle, R. J. Anthony, D. M. Kroll, U. R. Kortshagen, E. K. Hobbie, ACS Nano 2012, 6, 7389.
[65] A. Gupta, H. Wiggers, Phys. E Low-Dimens. Syst. Nanostructures 2009, 41, 1010.

[66] M. Dasog, G. B. De Los Reyes, L. V. Titova, F. A. Hegmann, J. G. C. Veinot, ACS Nano 2014, 8, 9636.

[67] K. Dohnalová, A. N. Poddubny, A. A. Prokofiev, W. D. A. M. de Boer, C. P. Umesh, J. M. Paulusse, H. Zuilhof, T. Gregorkiewicz, Light Sci. Appl. 2013, 2, e47.

[68] A. Gupta, M. T. Swihart, H. Wiggers, Adv. Funct. Mater. 2009, $19,696$.

[69] T. Nychyporuk, Y. Zakharko, V. Lysenko, M. Lemiti, Appl. Phys. Lett. 2009, 95, 083124.

[70] D. Mariotti, S. Mitra, V. Švrček, Nanoscale 2013, 5, 1385.

[71] M. C. Beard, K. P. Knutsen, P. Yu, J. M. Luther, O. Song, W. K. Metzger, R. J. Ellingson, A. J. Nozik, Nano Lett. 2007, 7, 2506.

[72] A. J. Nozik, Chem. Phys. Lett. 2008, 457, 3.

[73] A. J. Nozik, M. C. Beard, J. M. Luther, M. Law, R. J. Ellingson, J. C. Johnson, Chem. Rev. 2010, 110, 6873

[74] D. Timmerman, J. Valenta, K. Dohnalová, W. D. A. M. de Boer, T. Gregorkiewicz, Nat. Nanotechnol. 2011, 6, 710.

[75] M. T. Trinh, R. Limpens, W. D. A. M. de Boer, J. M. Schins, L. D. A. Siebbeles, T. Gregorkiewicz, Nat. Photonics 2012, 6, 316.

[76] G. Allan, C. Delerue, Phys. Rev. B 2008, 77, 125340.

[77] J.-W. Luo, A. Franceschetti, A. Zunger, Nano Lett. 2008, 8, 3174.

[78] S. A. Fischer, A. B. Madrid, C. M. Isborn, O. V. Prezhdo, J. Phys. Chem. Lett. 2010, 1, 232

[79] M. Govoni, I. Marri, S. Ossicini, Nat. Photonics 2012, 6, 672.

[80] D. Timmerman, I. Izeddin, P. Stallinga, I. N. Yassievich, T. Gregorkiewicz, Nat. Photonics 2008, 2, 105.

[81] J. J. H. Pijpers, M. T. W. Milder, C. Delerue, M. Bonn, J. Phys. Chem. C 2010, 114, 6318

[82] A. J. Nozik, Phys. E Low-Dimens. Syst. Nanostructures 2002, 14, 115.

[83] R. D. Schaller, V. M. Agranovich, V. I. Klimov, Nat. Phys. 2005, 1, 189.

[84] J. E. Murphy, M. C. Beard, A. G. Norman, S. P. Ahrenkiel, J. C. Johnson, P. Yu, O. I. Mićić, R. J. Ellingson, A. J. Nozik, J. Am. Chem. Soc. 2006, 128, 3241.

[85] R. J. Ellingson, M. C. Beard, J. C. Johnson, P. Yu, O. I. Micic, A. J. Nozik, A. Shabaev, A. L. Efros, Nano Lett. 2005, 5, 865.

[86] R. Schaller, V. Klimov, Phys. Rev. Lett. 2004, 92, 1.

[87] M. Wolf, R. Brendel, J. H. Werner, H. J. Queisser, J. Appl. Phys. 1998, 83, 4213.

[88] O. Jiang, S. H. Zhang, J. C. Li, Solid State Commun. 2004, $130,581$.

[89] A. A. Griffith, Philos. Trans. R. Soc. A Math. Phys. Eng. Sci. 1921, 221, 163.

[90] K. E. Aifantis, J. P. Dempsey, J. Power Sources 2005, 143, 203.

[91] J. R. Szczech, S. Jin, Energ. Environ. Sci. 2011, 4, 56.

[92] B. Fubini, V. Bolis, A. Cavenago, P. Ugliengo, J. Chem. Soc. Faraday Trans. 1992, 88, 277.

[93] A. M. Hartel, S. Gutsch, D. Hiller, C. Kübel, N. Zakharov, P. Werner, M. Zacharias, Appl. Phys. Lett. 2012, 101, 193103.

[94] L. Xu, Z. P. Li, C. Wen, W. Z. Shen, J. Appl. Phys. 2011, 110, 064315

[95] D. V. Talapin, C. B. Murray, Science 2005, 310, 86.

[96] M. Law, J. M. Luther, O. Song, B. K. Hughes, C. L. Perkins, A. J. Nozik, J. Am. Chem. Soc. 2008, 130, 5974.

[97] H. Kamiya, M. Iijima, Sci. Technol. Adv. Mater. 2010, 11, 044304.

[98] L. M. Wheeler, N. R. Neale, T. Chen, U. R. Kortshagen, Nat. Commun. 2013, 4, 2197. 
[99] M. Nie, D. P. Abraham, Y. Chen, A. Bose, B. L. Lucht, J. Phys. Chem. C 2013, 117, 13403.

[100] N. Delpuech, D. Mazouzi, N. Dupré, P. Moreau, M. Cerbelaud, J. S. Bridel, J.-C. Badot, E. De Vito, D. Guyomard, B. Lestriez, B. Humbert, J. Phys. Chem. C 2014, 118, 17318.

[101] R. N. Mehta, M. Chakraborty, P. A. Parikh, Int. J. Hydrogen Energ. 2014, 39, 8098.

[102] H. Bahruji, M. Bowker, P. R. Davies, Int. J. Hydrogen Energ. 2009, 34, 8504 .

[103] B. Goller, D. Kovalev, O. Sreseli, Nanotechnology 2011, 22, 305402.

[104] I. Oh, J. Kye, S. Hwang, Nano Lett. 2012, 12, 298.

[105] O. Khaselev, Science 1998, 280, 425.

[106] L. Schlapbach, A. Züttel, Nature 2001, 414, 353.

[107] A. Baldi, T. C. Narayan, A. L. Koh, J. A. Dionne, Nat. Mater. 2014, 1.

[108] M. Z. Jacobson, Science 2005, 308, 1901.

[109] N. Petermann, N. Stein, G. Schierning, R. Theissmann, B. Stoib, M. S. Brandt, C. Hecht, C. Schulz, H. Wiggers, J. Phys. D. Appl. Phys. 2011, 44, 174034.

[110] J. W. Sharp, S. J. Poon, H. J. Goldsmid, Phys. Status Solidi Appl. Res. 2001, 187, 507.

[111] Y. Oin, X. Wang, Z. L. Wang, Nature 2008, 451, 809.

[112] S. K. Bux, R. G. Blair, P. K. Gogna, H. Lee, G. Chen, M. S. Dresselhaus, R. B. Kaner, J.-P. Fleurial, Adv. Funct. Mater. 2009, 19, 2445.

[113] T. C. Harman, P. J. Taylor, M. P. Walsh, B. E. LaForge, Science 2002, 297, 2229.

[114] U. Kortshagen, J. Phys. D. Appl. Phys. 2009, 42, 113001.

[115] L. Mangolini, E. Thimsen, U. Kortshagen, Nano Lett. 2005, $5,655$.

[116] T. Lopez, L. Mangolini, J. Vac. Sci. Technol. B, Nanotechnol. Microelectron. Mater. Process. Meas. Phenom. 2014, 32, 061802.

[117] O. Yasar-Inceoglu, T. Lopez, E. Farshihagro, L. Mangolini, Nanotechnology 2012, 23, 255604.

[118] M. T. Swihart, J. Phys. Chem. A 2000, 104, 6083.

[119] U. Kortshagen, U. Bhandarkar, Phys. Rev. E 1999, 60, 887.

[120] U. Bhandarkar, U. Kortshagen, S. L. Girshick, J. Phys. D. Appl. Phys. 2003, 36, 1399.

[121] L. Mangolini, U. Kortshagen, Phys. Rev. E 2009, 79, 026405.

[122] F. Galli, U. R. Kortshagen, IEEE Trans. Plasma Sci. 2010, 38, 803.

[123] Z. C. Holman, U. R. Kortshagen, Nanotechnology 2010, 21 , 335302.

[124] O. Yasar-Inceoglu, L. Mangolini, Mater. Lett. 2013, 101, 76.

[125] R. P. Chaukulkar, K. de Peuter, J. A. Ghodes, S. Pylypenko, J. E. Cloud, Y. Yang, P. Stradins, S. Agarwal, Mater. Res. Express 2015, 2, 015019.

[126] D. Coleman, T. Lopez, O. Yasar-Inceoglu, L. Mangolini, J. Appl. Phys. 2015, 117, 193301.

[127] R. M. Roth, K. G. Spears, G. D. Stein, G. Wong, Appl. Phys. Lett. 1985, 46, 253.

[128] H. Kawasaki, H. Ohkura, T. Fukuzawa, M. Shiratani, Y. Watanabe, Y. Yamamoto, S. Suganuma, M. Hori, T. Goto, Jpn. J. Appl. Phys. 1997, 36, 4985.

[129] A. Bouchoule, A. Plain, L. Boufendi, J. P. Blondeau, C. Laure, J. Appl. Phys. 1991, 70, 1991.

[130] A. A. Howling, L. Sansonnens, J.-L. Dorier, C. Hollenstein, J. Appl. Phys. 1994, 75, 1340.

[131] M. Shiratani, H. Kawasaki, T. Fukuzawa, T. Yoshioka, Y. Ueda, S. Singh, Y. Watanabe, J. Appl. Phys. 1996, 79, 104.

[132] Y. Watanabe, J. Vac. Sci. Technol., A 1996, 14, 995.

[133] M. Shiratani, T. Fukuzawa, Y. Watanabe, Jpn. J. Appl. Phys. 1999, 38, 4542.
[134] T. Kakeya, K. Koga, M. Shiratani, Y. Watanabe, M. Kondo, Thin Solid Films 2006, 507, 288.

[135] S. Nunomura, M. Kita, K. Koga, M. Shiratani, Y. Watanabe, J. Appl. Phys. 2006, 99, 083302.

[136] K. Koga, S. Iwashita, M. Shiratani, J. Phys. D. Appl. Phys. 2007, 40, 2267.

[137] S. Nunomura, M. Shiratani, K. Koga, M. Kondo, Y. Watanabe, Phys. Plasmas 2008, 15, 080703.

[138] M. Shiratani, K. Koga, S. Iwashita, S. Nunomura, Faraday Discuss. 2008, 137, 127.

[139] M. Shiratani, K. Koga, S. Iwashita, G. Uchida, N. Itagaki, K. Kamataki, J. Phys. D. Appl. Phys. 2011, 44, 174038.

[140] A. Bouchoule, L. Boufendi, Plasma Sources Sci. Technol. 1993, 2, 204.

[141] A. A. Fridman, L. Boufendi, T. Hbid, B. V. Potapkin, A. Bouchoule, J. Appl. Phys. 1996, 79, 1303.

[142] L. Boufendi, M. C. Jouanny, E. Kovacevic, J. Berndt, M. Mikikian, J. Phys. D. Appl. Phys. 2011, 44, 174035.

[143] C. Hollenstein, J.-L. Dorier, J. Dutta, L. Sansonnens, A. A. Howling, Plasma Sources Sci. Technol. 1994, 3, 278.

[144] K. Kamataki, H. Miyata, K. Koga, G. Uchida, N. Itagaki, M. Shiratani, Appl. Phys. Express 2011, 4, 105001.

[145] T. Nozaki, K. Sasaki, T. Ogino, D. Asahi, K. Okazaki, Nanotechnology 2007, 18, 235603.

[146] T. Nozaki, K. Sasaki, T. Ogino, D. Asahi, K. Okazaki, J. Therm. Sci. Technol. 2007, 2, 192.

[147] R. M. Sankaran, D. Holunga, R. C. Flagan, K. P. Giapis, Nano Lett. 2005, 5, 537.

[148] W.-H. Chiang, C. Richmonds, R. M. Sankaran, Plasma Sources Sci. Technol. 2010, 19, 034011.

[149] V. Švrček, D. Mariott, M. Kondo, Appl. Phys. Lett. 2010, 97, 161502.

[150] C. Richmonds, R. M. Sankaran, Appl. Phys. Lett. 2008, 93, 2013.

[151] S. Ghosh, B. Bishop, I. Morrison, R. Akolkar, D. Scherson, M. R. Sankaran, J. Vac. Sci. Technol., A 2015, 33, 021312.

[152] B. Giesen, H. Wiggers, A. Kowalik, P. Roth, J. Nanoparticle Res. 2005, 7, 29.

[153] N. Rao, S. Girshick, J. Heberlein, P. McMurry, S. Jones, D. Hansen, B. Micheel, Plasma Chem. Plasma Process. 1995, 15, 581.

[154] N. P. Rao, H. J. Lee, M. Kelkar, D. J. Hansen, J. V. R. Heberlein, P. H. McMurry, S. L. Girshick, Nanostructured Mater. 1997, 9, 129.

[155] N. P. Rao, N. Tymiak, J. Blum, A. Neuman, H. J. Lee, S. L. Girshick, P. H. McMurry, J. Heberlein, J. Aerosol Sci. 1998, 29, 707.

[156] V. Colombo, C. Deschenaux, E. Ghedini, M. Gherardi, C. Jaeggi, M. Leparoux, V. Mani, P. Sanibondi, Plasma Sources Sci. Technol. 2012, 21, 045010.

[157] M. Kambara, A. Kitayama, K. Homma, T. Hideshima, M. Kaga, K.-Y. Sheem, S. Ishida, T. Yoshida, J. Appl. Phys. 2014, 115, 143302.

[158] S. L. Girshick, C.-P. Chiu, Plasma Chem. Plasma Process. 1989, 9, 355.

[159] S. L. Girshick, Plasma Sources Sci. Technol. 1994, 3, 388.

[160] V. Colombo, E. Ghedini, M. Gherardi, P. Sanibondi, M. Shigeta, Plasma Sources Sci. Technol. 2012, 21, 025001.

[161] V. Colombo, E. Ghedini, M. Gherardi, P. Sanibondi, Plasma Sources Sci. Technol. 2012, 21, 055007.

[162] V. Colombo, E. Ghedini, M. Gherardi, P. Sanibondi, Plasma Sources Sci. Technol. 2013, 22, 035010.

[163] B. M. Goortani, P. Proulx, S. Xue, N. Y. Mendoza-Gonzalez, Powder Technol. 2007, 175, 22. 
[164] M. Kambara, N. Oda, K. Homma, Jpn. J. Appl. Phys. 2015, 54, 01AD05.

[165] T. Tashiro, M. Kaga, M. Kambara, Jpn. J. Appl. Phys. 2015, 54, $01 \mathrm{AD} 03$.

[166] M. Leparoux, C. Schreuders, J.-W. Shin, S. Siegmann, Adv. Eng Mater. 2005, 7, 349.

[167] T. Ishigaki, S.-M. Oh, J.-G. Li, D.-W. Park, Sci. Technol. Adv. Mater. 2005, 6, 111

[168] J. W. Shin, H. Miyazoe, M. Leparoux, S. Siegmann, J. L. Dorier, C. Hollenstein, Plasma Sources Sci. Technol. 2006, 15, 441.

[169] J.-G. Li, H. Kamiyama, X.-H. Wang, Y. Moriyoshi, T. Ishigaki, J. Eur. Ceram. Soc. 2006, 26, 423.

[170] M. Mamak, S. Y. Choi, U. Stadler, R. Dolbec, M. Boulos, S. Petrov, J. Mater. Chem. 2010, 20, 9855.

[171] W. M. M. Kessels, J. P. M. Hoefnagels, M. G. H. Boogaarts, D. C. Schram, M. C. M. van de Sanden, J. Appl. Phys. 2001, 89, 2065.

[172] M. C. M. van de Sanden, R. J. Severens, W. M. M. Kessels, R. F. G. Meulenbroeks, D. C. Schram, J. Appl. Phys. 1998, 84, 2426.

[173] W. M. M. Kessels, A. H. M. Smets, M. C. M. van de Sanden, J. Non. Cryst. Solids 2004, 338-340, 27.

[174] J. W. A. M. Gielen, W. M. M. Kessels, M. C. M. van de Sanden, D. C. Schram, J. Appl. Phys. 1997, 82, 2643.

[175] B. Hoex, F. J. J. Peeters, M. Creatore, M. D. Bijker, W. M. M. Kessels, M. C. M. van de Sanden, Conf. Rec. IEEE 4th World Conf. Photovolt. Energy Convers. 2006, 1, 1134.

[176] W. M. M. Kessels, J. Hong, F. J. H. van Assche, J. D. Moschner, T. Lauinger, W. J. Soppe, A. W. Weeber, D. C. Schram, M. C. M. van de Sanden, J. Vac. Sci. Technol., A 2002, 20, 1704.

[177] A. de Graaf, G. Dinescu, J. L. Longueville, M. C. M. van de Sanden, D. C. Schram, E. H. A. Dekempeneer, L. J. van Ijzendoorn, Thin Solid Films 1998, 333, 29.

[178] R. Groenen, J. L. Linden, H. R. M. van Lierop, D. C. Schram, A. D. Kuypers, M. C. M. van de Sanden, Appl. Surf. Sci. 2001, $173,40$.

[179] J. J. Beulens, M. J. de Graaf, D. C. Schram, Plasma Sources Sci. Technol. 1993, 2, 180.

[180] M. C. M. van de Sanden, J. M. de Regt, D. C. Schram, Phys. Rev. E 1993, 47, 2792.

[181] R. Engeln, S. Mazouffre, P. Vankan, D. C. Schram, N. Sadeghi, Plasma Sources Sci. Technol. 2001, 10, 595.

[182] W. M. M. Kessels, C. M. Leewis, A. Leroux, M. C. M. van de Sanden, D. C. Schram, J. Vac. Sci. Technol., A 1999, 17, 1531.

[183] W. M. M. Kessels, M. C. M. van de Sanden, D. C. Schram, Appl. Phys. Lett. 1998, 72, 2397.

[184] W. M. M. Kessels, C. M. Leewis, M. C. M. van de Sanden, D. C. Schram, J. Appl. Phys. 1999, 86, 4029.

[185] J. Perrin, O. Leroy, M. C. Bordage, Contrib. to Plasma Phys. 1996, 36, 3.

[186] W. M. M. Kessels, M. C. M. van de Sanden, D. C. Schram, J. Vac Sci. Technol., A 2000, 18, 2153.

[187] İ. Doğan, M. C. M. van de Sanden, J. Appl. Phys. 2013, 114, 134310.

[188] F. Erogbogbo, K. Yong, I. Roy, G. Xu, ACS Nano 2008, 2, 873.

[189] X. Pi, T. Yu, D. Yang, Part. Part. Syst. Charact. 2014, 31, 751.

[190] J. G. C. Veinot, Chem. Commun. (Camb). 2006, 4160.

[191] R. Wang, X. Pi, D. Yang, J. Phys. Chem. C 2012, 116, 19434.

[192] D. Mariotti, R. M. Sankaran, J. Phys. D. Appl. Phys. 2010, 43, 323001.

[193] İ. Doğan, R. Gresback, T. Nozaki, M. C. M. van de Sanden, preprint arXiv 2014, 1412, 8622.

[194] R. J. Anthony, K.-Y. Cheng, Z. C. Holman, R. J. Holmes, U. R. Kortshagen, Nano Lett. 2012, 12, 2822.
[195] J. Nelles, D. Sendor, A. Ebbers, F. M. Petrat, H. Wiggers, C. Schulz, U. Simon, Colloid Polym. Sci. 2007, 285, 729.

[196] X. Pi, L. Zhang, D. Yang, J. Phys. Chem. C 2012, 116, 21240.

[197] T. Chen, B. Skinner, W. Xie, B. I. Shklovskii, U. R. Kortshagen, J. Phys. Chem. C 2014, 118, 19580.

[198] I. E. Anderson, R. A. Shircliff, C. Macauley, D. K. Smith, B. G. Lee, S. Agarwal, P. Stradins, R. T. Collins, J. Phys. Chem. C 2012, 116, 3979.

[199] W. Shockley, H. J. Queisser, J. Appl. Phys. 1961, 32, 510.

[200] G. F. Brown, J. Wu, Laser Photonics Rev. 2009, 3, 394.

[201] L. Khriachtchev, S. Ossicini, F. Iacona, F. Gourbilleau, Int. J. Photoenerg. 2012, 2012, 1.

[202] G. Conibeer, Mater. Today 2007, 10, 42.

[203] P. Ingenhoven, A. Anopchenko, A. Tengattini, D. Gandolfi, F. Sgrignuoli, G. Pucker, Y. Jestin, L. Pavesi, R. Balboni, Phys. status solidi 2013, 210, 1071.

[204] F. Meillaud, A. Shah, C. Droz, E. Vallat-Sauvain, C. Miazza, Sol. Energ. Mater. Sol. Cells 2006, 90, 2952.

[205] V. Švrček, A. Slaoui, J. C. Muller, Thin Solid Films 2004, 451-452, 384.

[206] Z. Yuan, G. Pucker, A. Marconi, F. Sgrignuoli, A. Anopchenko, Y. Jestin, L. Ferrario, P. Bellutti, L. Pavesi, Sol. Energ. Mater. Sol. Cells 2011, 95, 1224.

[207] F. Sgrignuoli, G. Paternoster, A. Marconi, P. Ingenhoven, A. Anopchenko, G. Pucker, L. Pavesi, J. Appl. Phys. 2012, 111, 034303.

[208] Y. Kawashima, K. Yamamoto, M. Sato, K. Nakahara, T. Matsunaga, W. M. Nakamura, D. Yamashita, H. Matsuzaki, G. Uchida, K. Kamataki, N. Itagaki, K. Koga, M. Shiratani, M. Kondo, Photovoltaic Specialists Conference (PVSC), 2010 35th IEEE, 2010, 3347.

[209] G. Uchida, Y. Kawashima, K. Yamamoto, M. Sato, K. Nakahara, T. Matsunaga, D. Yamashita, H. Matsuzaki, K. Kamataki, N. Itagaki, K. Koga, M. Kondo, M. Shiratani, Phys. Status Solidi Curr. Top. Solid State Phys. 2011, 8, 3021.

[210] H. Seo, Y. Wang, M. Sato, G. Uchida, K. Kamataki, N. Itagaki, K. Koga, M. Shiratani, Jpn. J. Appl. Phys. 2013, 52, D05.

[211] H. Seo, Y. Wang, M. Sato, G. Uchida, K. Koga, N. Itagaki, K. Kamataki, M. Shiratani, Thin Solid Films 2013, 546, 284.

[212] G. Uchida, M. Sato, H. Seo, K. Kamataki, N. Itagaki, K. Koga, M. Shiratani, Thin Solid Films 2013, 544, 93.

[213] H. Seo, M. Son, H. Kim, Y. Wang, G. Uchida, K. Kamataki, N. Itagaki, K. Koga, M. Shiratani, Jpn. J. Appl. Phys. 2013, 52, $10 \mathrm{MB} 07$.

[214] H. Seo, D. Ichida, G. Uchida, K. Kamataki, N. Itagaki, K. Koga, M. Shiratani, Int. J. Precis. Eng. Manuf. 2014, 15, 339.

[215] H. Seo, D. Ichida, S. Hashimoto, G. Uchida, N. Itagaki, K. Koga, M. Shiratani, Jpn. J. Appl. Phys. 2015, 54, D02.

[216] G. Uchida, Y. Wang, D. Ichida, H. Seo, K. Kamataki, N. Itagaki, K. Koga, M. Shiratani, Jpn. J. Appl. Phys. 2013, 52, A05.

[217] Y.-L. Lee, C.-H. Chang, J. Power Sources 2008, 185, 584.

[218] M.-H. Yeh, C.-P. Lee, C.-Y. Chou, L.-Y. Lin, H.-Y. Wei, C.-W. Chu, R. Vittal, K.-C. Ho, Electrochim. Acta 2011, 57, 277.

[219] H. Seo, Y. Wang, G. Uchida, K. Kamataki, N. Itagaki, K. Koga, M. Shiratani, Electrochim. Acta 2013, 95, 43.

[220] C.-Y. Liu, Z. C. Holman, U. R. Kortshagen, Nano Lett. 2009, 9, 449.

[221] R. W. Liptak, J. Yang, N. J. Kramer, U. Kortshagen, S. A. Campbell, Nanotechnology 2012, 23, 395205.

[222] Y. Adjallah, C. Anderson, U. Kortshagen, J. Kakalios, J. Appl. Phys. 2010, 107, 043704.

[223] W. R. Taube, A. Kumar, R. Saravanan, P. B. Agarwal, P. Kothari, B. C. Joshi, D. Kumar, Sol. Energ. Mater. Sol. Cells 2012, 101, 32. 
[224] D. Herrmann, S. Niesar, C. Scharsich, A. Köhler, M. Stutzmann, E. Riedle, J. Am. Chem. Soc. 2011, 133, 18220.

[225] Y. Ding, R. Gresback, R. Yamada, K. Okazaki, T. Nozaki, Jpn. J. Appl. Phys. 2013, 52, 11NM04.

[226] R. Marom, S. F. Amalraj, N. Leifer, D. Jacob, D. Aurbach, J. Mater. Chem. 2011, 21, 9938.

[227] H. Li, Z. Wang, L. Chen, X. Huang, Adv. Mater. 2009, 21, 4593.

[228] M. N. Obrovac, L. Christensen, Electrochem. Solid-State Lett. 2004, 7, A93.

[229] M. N. Obrovac, V. L. Chevrier, Chem. Rev. 2014, 114, 11444.

[230] J. P. Maranchi, A. F. Hepp, A. G. Evans, N. T. Nuhfer, P. N. Kumta, J. Electrochem. Soc. 2006, 153, A1246.

[231] M. Park, M. Kim, J. Joo, K. Kim, J. Kim, S. Ahn, Nano Lett. 2009, 9, 3844.

[232] L. Hu, H. Wu, S. S. Hong, L. Cui, J. R. McDonough, S. Bohy, Y. Cui, Chem. Commun. (Camb). 2011, 47, 367.

[233] Y. Yu, L. Gu, C. Zhu, S. Tsukimoto, P. A. van Aken, J. Maier, Adv. Mater. 2010, 22, 2247.

[234] C. Martin, M. Alias, F. Christien, O. Crosnier, D. Belanger, T. Brousse, Adv. Mater. 2009, 21, 4735.

[235] T. Zhang, J. Gao, H. P. Zhang, L. C. Yang, Y. P. Wu, H. O. Wu, Electrochem. commun. 2007, 9, 886.

[236] T. H. Hwang, Y. M. Lee, B.-S. Kong, J.-S. Seo, J. W. Choi, Nano Lett. 2012, 12, 802.

[237] Y. Kwon, G.-S. Park, J. Cho, Electrochim. Acta 2007, 52, 4663.

[238] C. Kim, M. Noh, M. Choi, J. Cho, B. Park, Chem. Mater. 2005, 17, 3297.

[239] D. S. Jung, T. H. Hwang, S. Bin Park, J. W. Choi, Nano Lett. 2013, 13, 2092.

[240] D. E. Arreaga-Salas, A. K. Sra, K. Roodenko, Y. J. Chabal, C. L. Hinkle, J. Phys. Chem. C 2012, 116, 9072.

[241] J. L. Gómez Cámer, J. Morales, L. Sánchez, P. Ruch, S. H. Ng, R. Kötz, P. Novák, Electrochim. Acta 2009, 54, 6713.

[242] H. Wolf, Z. Pajkic, T. Gerdes, M. Willert-Porada, J. Power Sources 2009, 190, 157.

[243] J. H. Byeon, Y.-W. Kim, Adv. Mater. Interfaces 2014, 1, 1300134.

[244] B. Jang, J. Kim, J. Lee, Phys. Status Solidi 2014, 211, 1752.

[245] B. J. Jeon, J. K. Lee, J. Alloys Compd. 2014, 590, 254.

[246] Y.-S. Na, S. Choi, D.-W. Park, Phys. Status Solidi 2014, 211, 2749.

[247] K. Homma, M. Kambara, T. Yoshida, Sci. Technol. Adv. Mater. 2014, 15, 025006.

[248] X. Chang, W. Li, J. Yang, L. Xu, J. Zheng, X. Li, J. Mater. Chem. A 2015, 3, 3522.

[249] C. Jung, J. Koo, B. Jang, J. Kim, J. Lee, S. Kim, M. Han, Thin Solid Films 2015, 587, 142.

[250] D. Leblanc, C. Wang, Y. He, D. Bélanger, K. Zaghib, J. Power Sources 2015, 279, 522.

[251] L. Zhong, J. Guo, L. Mangolini, J. Power Sources 2015, 273, 638.

[252] S. Litvinenko, S. Alekseev, V. Lysenko, A. Venturello, F. Geobaldo, L. Gulina, G. Kuznetsov, V. Tolstoy, V. Skryshevsky, E. Garrone, Int. J. Hydrogen Energ. 2010, 35, 6773.

[253] J. Oh, T. G. Deutsch, H.-C. Yuan, H. M. Branz, Energ. Environ. Sci. 2011, 4, 1690.
[254] S. Y. Reece, J. A. Hamel, K. Sung, T. D. Jarvi, A. J. Esswein, J. J. H. Pijpers, D. G. Nocera, Science 2011, 334, 645.

[255] C. E. Bunker, M. J. Smith, J. Mater. Chem. 2011, 21, 12173.

[256] X. Ma, M. R. Zachariah, Int. J. Hydrogen Energ. 2010, 35, 2268.

[257] C. Zhan, P. K. Chu, D. Ren, Y. Xin, K. Huo, Y. Zou, N. K. Huang, Int. J. Hydrogen Energ. 2011, 36, 4513.

[258] J. P. Dismukes, L. Ekstrom, E. F. Steigmeier, I. Kudman, D. S. Beers, J. Appl. Phys. 1964, 35, 2899.

[259] X. D. Pi, U. Kortshagen, Nanotechnology 2009, 20, 295602.

[260] D. J. Rowe, U. R. Kortshagen, APL Mater. 2014, 2, 022104.

[261] G. Joshi, H. Lee, Y. Lan, X. Wang, G. Zhu, D. Wang, R. W. Gould, D. C. Cuff, M. Y. Tang, M. S. Dresselhaus, G. Chen, Z. Ren, Nano Lett. 2008, 8, 4670.

[262] X. W. Wang, H. Lee, Y. C. Lan, G. H. Zhu, G. Joshi, D. Z. Wang, J. Yang, A. J. Muto, M. Y. Tang, J. Klatsky, S. Song, M. S. Dresselhaus, G. Chen, Z. F. Ren, Appl. Phys. Lett. 2008, 93, 193121.

[263] A. A. Usenko, D. O. Moskovskikh, M. V. Gorshenkov, A. V. Korotitskiy, S. D. Kaloshkin, A. I. Voronin, V. V. Khovaylo, Scr. Mater. 2015, 96, 9.

[264] S. Chen, X. Zhang, W. Fan, T. Yi, D. V. Quach, S. Bux, O. Meng, S. M. Kauzlarich, Z. A. Munir, J. Alloys Compd. 2015, 625, 251.

[265] G. Bernard-Granger, M. Soulier, H. Ihou-Mouko, C. Navone, M. Boidot, J. Leforestier, J. Simon, J. Alloys Compd. 2015, 618, 403.

[266] X. D. Pi, R. Gresback, R. W. Liptak, S. A. Campbell, U. Kortshagen, Appl. Phys. Lett. 2008, 92, 123102.

[267] S. Zhou, X. Pi, Z. Ni, O. Luan, Y. Jiang, C. Jin, T. Nozaki, D. Yang, Part. Part. Syst. Charact. 2015, 32, 213.

[268] Z. Ni, X. Pi, M. Ali, S. Zhou, T. Nozaki, D. Yang, J. Phys. D. Appl. Phys. 2015, 48, 314006.

[269] S. P. Ashby, J. A. Thomas, J. García-Cañadas, G. Min, J. Corps, A. V. Powell, H. Xu, W. Shen, Y. Chao, Faraday Discuss. 2014, $176,349$.

[270] E. Drahi, A. Gupta, S. Blayac, S. Saunier, P. Benaben, Phys. status solidi 2014, 211, 1301.

[271] T. Claudio, N. Stein, D. G. Stroppa, B. Klobes, M. M. Koza, P. Kudejova, N. Petermann, H. Wiggers, G. Schierning, R. P. Hermann, Phys. Chem. Chem. Phys. 2014, 16, 25701.

[272] N. Petermann, T. Schneider, J. Stötzel, N. Stein, C. Weise, I. Wlokas, G. Schierning, H. Wiggers, J. Phys. D. Appl. Phys. 2015, 48, 314010.

[273] A. Miura, S. Zhou, T. Nozaki, J. Shiomi, ACS Appl. Mater. Interface. 2015, 7, 13484.

[274] S. L. Girshick, C.-P. Chiu, P. H. McMurry, Aerosol Sci. Technol. 1990, 13, 465.

[275] S. J. Choi, M. J. Kushner, J. Appl. Phys. 1993, 74, 853.

[276] T. J. Sommerer, M. S. Barnes, J. H. Keller, M. J. McCaughey, M. J. Kushner, Appl. Phys. Lett. 1991, 59, 638.

[277] S. J. Choi, M. J. Kushner, IEEE Trans. Plasma Sci. 1994, 22, 138.

[278] S. J. Choi, M. J. Kushner, J. Appl. Phys. 1994, 75, 3351.

[279] M. J. McCaughey, M. J. Kushner, Appl. Phys. Lett. 1989, $55,951$. 